\title{
Stochastic Modeling and Analysis of Multichannel SW-ARQ
}

by

Jun Li

\author{
A thesis submitted to \\ the Faculty of Graduate Studies and Research \\ in partial fulfillment of the requirements for the degree of
}

\section{Doctor of Philosophy}

School of Mathematics and Statistics

\author{
Carleton University \\ Ottawa, Ontario, Canada
}

September, 2008

(C) Copyright 2008

Jun $\mathrm{Li}$ 


$\begin{array}{ll}\begin{array}{l}\text { Library and } \\ \text { Archives Canada }\end{array} & \begin{array}{l}\text { Bibliothèque et } \\ \text { Archives Canada }\end{array} \\ \begin{array}{l}\text { Published Heritage } \\ \text { Branch }\end{array} & \begin{array}{l}\text { Direction du } \\ \text { Patrimoine de l'édition }\end{array} \\ \begin{array}{l}\text { 395 Wellington Street } \\ \text { Ottawa ON K1A ON4 } \\ \text { Canada }\end{array} & \begin{array}{l}\text { 395, rue Wellington } \\ \text { Ottawa ON K1A 0N4 } \\ \text { Canada }\end{array}\end{array}$

Yourfile Votre référence ISBN: 978-0-494-43899-2

Our file Notre référence

ISBN: 978-0-494-43899-2

NOTICE:

The author has granted a nonexclusive license allowing Library and Archives Canada to reproduce, publish, archive, preserve, conserve, communicate to the public by telecommunication or on the Internet, loan, distribute and sell theses worldwide, for commercial or noncommercial purposes, in microform, paper, electronic and/or any other formats.

The author retains copyright ownership and moral rights in this thesis. Neither the thesis nor substantial extracts from it may be printed or otherwise reproduced without the author's permission.
AVIS:

L'auteur a accordé une licence non exclusive permettant à la Bibliothèque et Archives Canada de reproduire, publier, archiver, sauvegarder, conserver, transmettre au public par télécommunication ou par l'Internet, prêter, distribuer et vendre des thèses partout dans le monde, à des fins commerciales ou autres, sur support microforme, papier, électronique et/ou autres formats.

L'auteur conserve la propriété du droit d'auteur et des droits moraux qui protège cette thèse. $\mathrm{Ni}$ la thèse ni des extraits substantiels de celle-ci ne doivent être imprimés ou autrement reproduits sans son autorisation.
In compliance with the Canadian

Privacy Act some supporting forms may have been removed from this thesis.

While these forms may be included in the document page count, their removal does not represent any loss of content from the thesis.
Conformément à la loi canadienne sur la protection de la vie privée, quelques formulaires secondaires ont été enlevés de cette thèse.

Bien que ces formulaires aient inclus dans la pagination, il n'y aura aucun contenu manquant.

\section{Canada}


Stochastic Modeling and Analysis of Multichannel SW-ARQ

\section{JUN LI}

2008 


\begin{abstract}
With rapid advances in wireless communication and networking, orthogonal frequency division multiplexing (OFDM) and multiple-input multiple-output antennas (MIMO) are often adopted in communication systems to create multiple parallel channels. To implement error control through retransmission of packets in a multichannel communication system, Automatic-Repeat-reQuest (ARQ) schemes have been generalized for parallel multichannel data communications. In this thesis, we develop two stochastic systems, denoted by MSW-ARQ and MSW-ARQ-inS, for modeling stop-and-wait ARQ over multiple channels with either time-invariant or time-varying error rates. We evaluate their performance analytically and through simulation. With a time-invariant error rate for each channel, we derive the distribution function of the packet delay for both stochastic systems. Meanwhile, we analyze the probability generating function of the resequencing buffer occupancy and the distribution function of the resequencing delay for MSW-ARQinS. By assuming a time-varying error model, we obtain statistical properties of the resequencing buffer occupancy and the resequencing delay for MSW-ARQ-inS. Based on results from the analysis, we numerically compute the probability mass functions of the resequencing buffer occupancy, the resequencing delay, and the packet delay for selected values of system parameters, from which the system performance is evaluated. From numerical and simulation results, we identify trends in the mean resequencing buffer occupancy, the mean resequencing delay and the mean packet delay as functions of system parameters. We confirm that the optimal packet transmission scheduling under the throughput standard is also optimal under the delay criterion. We expect that the modeling technique and analysis approach proposed in this thesis can be applied to the performance evaluation of other ARQ protocols (e.g., selective-repeat ARQ) over multiple channels.
\end{abstract}




\section{Acknowledgements}

I am sincerely grateful to my supervisor Dr. Yiqiang Q. Zhao for his invaluable guidance, enlightening comments and constant encouragement throughout my studies at Carleton University. His profound knowledge and remarkable research expertise have significantly benefited my academic growth. Working with him has always been an enjoyable and rewarding experience. I would like to acknowledge the participation in discussions of my former co-supervisor, the late Professor Amit Bose, and the education provided by the School of Mathematics and Statistics, Carleton University. My thanks go to the faculty members and staff of the School and the Faculty of Graduate Studies and Research, who helped me during my studies at Carleton University, especially Dr. P. Farrell, Dr. M. Huang, Dr. M. Ould Haye, Dr. G. Park, Ms. V. Daley, and Ms. B. Nicholl.

I would like to acknowledge the support in part from the following programs for this research: the Ontario Graduate Scholarship (OGS) Program, CANCCOM Project of MITACS, NSERC Discovery Grants, the MITACS-Alcatel-Lucent Graduate Internship Program, and the Graduate Research Assistantship Program at CRC.

Many thanks also go to my friends Mr. F. Larochelle, Dr. G. Li, Dr. B. Liu, Dr. Y. Liu, Mr. K. Oliver and Mr. P. Rabinovitch for their useful discussions about my research.

Finally, I thank my parents and my sisters, who are currently in China, for their encouragement. I would like to dedicate this thesis to my wife, Claudia X. Li, and our lovely child Jeffrey. I could not have completed this thesis without their love. 


\section{Glossary}

Automatic-Repeat-reQuest (or ARQ): An error-correction technique in digital data transmission through data retransmission. Three ARQ schemes, which are SW-ARQ, GBN-ARQ, and SR-ARQ, have been developed.

Channel Model: A channel model is a probabilistic model characterizing the statistical property of transmission errors of packets when being transmitted over a channel.

iid Model: The iid model defines a time-invariant channel model, in which the transmission error of a packet is described by a fixed error rate in any time slot.

Markov Model: The Markov model defines a time-varying channel model. In this model, the transmission error of a packet is modulated by a two-state Markov chain.

Multichannel System: A data transmission system where the transmitter and the receiver are connected through a link that consists of multiple parallel (forward) channels. In this thesis, the number of parallel channels are assumed to be $M$.

Packet Delay: Packet delay is a performance metric evaluated in this thesis. It is measured by the number of the time units (or slots) from the epoch of the first-time transmission until the epoch of leaving the system for an arbitrary packet.

Packet Scheduling Policy: A packet scheduling policy is a packet-to-channel assignment rule which the transmitter uses to simultaneously transmit multiple packets over parallel channels. In the static scheduling policy, a packet to be retransmitted 
is always assigned to the same channel as originally assigned one, while, in the dynamic scheduling policy, a packet of the $k^{\text {th }}$ smallest sequence number in the block of packets to be transmitted is assigned to the channel having the $k^{\text {th }}$ smallest error rate.

Round Trip Time (or RTT): The period from the instant at which a packet is sent out by the transmitter until the time at which its acknowledgement arrives at the transmitter. In this thesis, time is slotted, and RTT is assumed to be an even integer number, denoted by $m-1$ slots.

Resequencing Buffer Occupancy: Resequencing buffer occupancy is a performance metric measured by the number of packets buffered at the resequencing buffer at the receiver.

Resequencing Delay: Resequencing delay is a performance metric measured by the number of the time units (or slots), with which an arbitrary packet has to spend in the resequencing buffer. 


\section{Contents}

Title Page $\quad$ i

Acceptance Sheet ii

Abstract

Acknowledgements iv

Glossary v

Contents vii

1 Introduction 1

1.1 Background ........................... 1

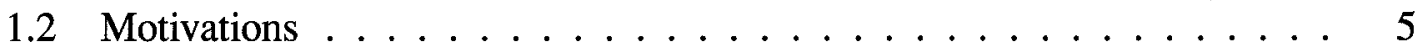

1.3 Contributions .................... 8

1.4 Thesis Outline . . . . . . . . . . . . . . . . . 10

2 Multichannel Systems with ARQ $\quad 11$

2.1 A Multichannel System with ARQ Error Control . . . . . . . . . . 11 
2.2 Channel Models . . . . . . . . . . . . . . . . . . . . . . . . . 13

2.2 .1 The iid Model . . . . . . . . . . . . . . . . . 13

2.2.2 The Markov Model . . . . . . . . . . . . . . . . . . 13

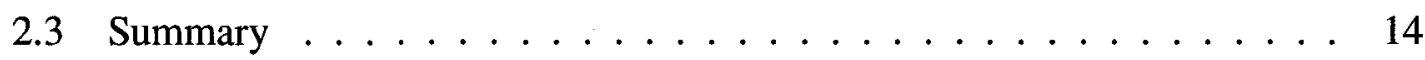

3 Multichannel SW-ARQ Models 15

3.1 Multichannel SW-ARQ Models $\ldots \ldots \ldots \ldots \ldots \ldots$

$3.1 .1 \quad$ MSW-ARQ $\ldots \ldots \ldots \ldots \ldots \ldots \ldots \ldots \ldots$

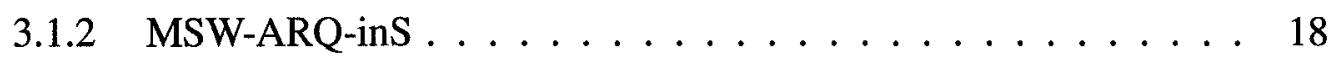

3.2 Study Objective and Performance Metrics _. . . . . . . . . . 19

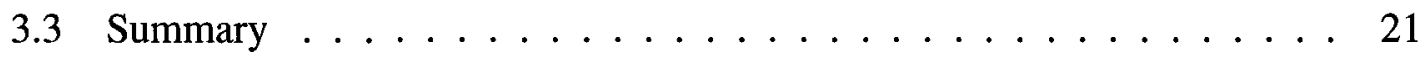

4 Resequencing Analysis of MSW-ARQ-inS 22

4.1 Analysis for Time-invariant Channels . . . . . . . . . 23

4.1.1 Resequencing Buffer Occupancy . . . . . . . . . . . 26

4.1.2 Resequencing Delay . . . . . . . . . . . . . . . . . . 29

4.2 Analysis for Markov Channels . . . . . . . . . . . . . . . 34

4.3 Numerical and Simulation Results . . . . . . . . . . . . . 43

4.3.1 Distribution Functions for Time-invariant Channels . . . . . . . 43

4.3.2 Means for Time-invariant Channels . . . . . . . . . . . 46

4.3.3 Means for Markov Channels . . . . . . . . . . . . . 50

4.4 Summary $\ldots \ldots \ldots \ldots \ldots \ldots \ldots \ldots \ldots \ldots \ldots$

5 Packet Delay Analysis $\quad 57$

5.1 Packet Delay of MSW-ARQ $\ldots \ldots \ldots \ldots \ldots \ldots$

viii 
5.2 Packet Delay of MSW-ARQ-inS . . . . . . . . . . . . . 63

5.3 Numerical and Simulation Results . . . . . . . . . . . . . . . 64

5.3.1 Distribution Function of Packet Delay . . . . . . . . . . . 65

5.3.2 Performance Comparison of MSW-ARQ and MSW-ARQ-inS . . 67

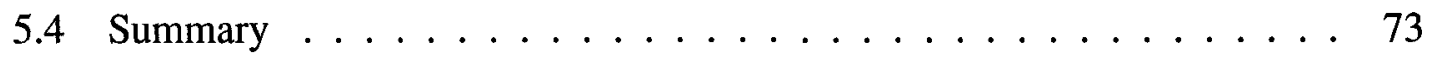

6 Conclusion and Future Work $\quad 74$

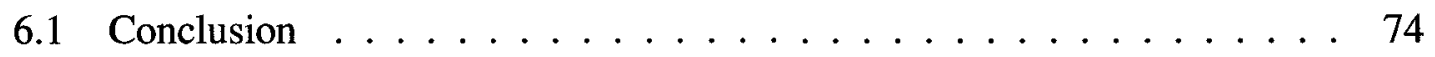

6.2 Future Work ........................ 75

$\begin{array}{ll}\text { Appendix } & \mathbf{7 6}\end{array}$

$\begin{array}{lll}\text { A Literature on Packet Resequencing } & 76\end{array}$

$\begin{array}{lr}\text { Bibliography } & \mathbf{8 0}\end{array}$ 


\section{List of Figures}

2.1 A Multichannel System with ARQ . . . . . . . . . . . . 12

3.1 MSW-ARQ $(\mathrm{M}=3, \mathrm{~m}=5) \ldots \ldots \ldots \ldots \ldots \ldots \ldots$

3.2 MSW-ARQ-inS $(\mathrm{M}=3, \mathrm{~m}=5) \ldots \ldots \ldots \ldots \ldots$

4.1 Processes $\left\{X_{i}(t): t \geq 0\right\}$ and $\left\{Y_{i}(t): t \geq 0\right\}(\mathrm{M}=3, \mathrm{~m}=5) \ldots \ldots 24$

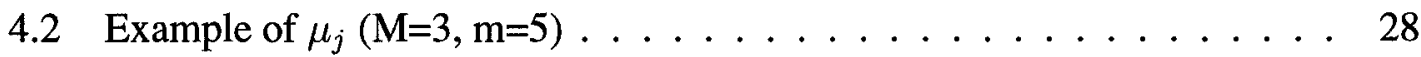

4.3 The Probability Mass Function of $B_{r} \ldots \ldots \ldots \ldots \ldots$

4.4 The Probability Mass Function of $D_{r} \ldots \ldots \ldots \ldots \ldots \ldots$

4.5 The Probability Mass Function of $D_{r} \ldots \ldots \ldots \ldots \ldots \ldots$

4.6 Average Resequencing Buffer Occupancy vs. $M \ldots \ldots \ldots$

4.7 Average Resequencing Buffer Occupancy vs. $\Delta \ldots \ldots \ldots \ldots$. . . . . 49

4.8 Mean Resequencing Delay $\ldots \ldots \ldots$. . . . . . . . . . . 50

4.9 Average Resequencing Buffer Occupancy vs. $K \ldots \ldots \ldots \ldots \ldots$

4.10 Average Resequencing Buffer Occupancy vs. $\bar{p} \ldots \ldots \ldots \ldots$

4.11 Average Resequencing Buffer Occupancy vs. $M \ldots \ldots \ldots \ldots$

4.12 Mean Resequencing Delay $\ldots \ldots \ldots \ldots$

5.1 Process $\left\{X_{3}(t): t \geq 0\right\}(\mathrm{M}=3, \mathrm{~m}=5) \ldots \ldots \ldots \ldots \ldots \ldots$ 
5.2 The pmf of $D$ vs. $M(\Delta=1.2, p=0.25) \ldots \ldots \ldots \ldots$

5.3 The pmf of $D$ vs. $p(M=8, \Delta=1.2) \ldots \ldots \ldots \ldots \ldots$

5.4 The pmf of $D$ vs. $\Delta(M=8, p=0.25) \ldots \ldots \ldots \ldots \ldots$

5.5 The pmf of $\bar{D}$ vs. $M(\Delta=1.2, p=0.25) \ldots \ldots \ldots \ldots \ldots 7$

5.6 The pmf of $\bar{D}$ vs. $p(M=8, \Delta=1.2) \ldots \ldots \ldots \ldots$

5.7 The pmf of $\bar{D}$ vs. $\Delta(M=8, p=0.25) \ldots \ldots \ldots \ldots$

5.8 Average Packet Delay vs. $M(\Delta=1.2, p=0.25) \ldots \ldots \ldots \ldots$

5.9 Average Packet Delay vs. $p(\Delta=1.2, M=8) \ldots \ldots \ldots \ldots \ldots$

5.10 Average Packet Delay vs. $\Delta(M=8, p=0.25) \ldots \ldots \ldots \ldots 71$

5.11 Average Packet Delay for Time-Varying Channels . . . . . . . . . 72

A.1 A General Resequencing Network . . . . . . . . . . . . . 77

A.2 The Disordering Network with Finite Servers $\ldots \ldots \ldots \ldots$

A.3 The Disordering Network with Switched Input . . . . . . . . . . . 79 


\section{List of Tables}




\section{Chapter 1}

\section{Introduction}

In this chapter, background and motivations of this thesis research are elaborated in Section 1.1 and Section 1.2, respectively. The main research contributions are listed in Section 1.3, followed by the thesis outline stated in Section 1.4.

\subsection{Background}

Automatic-Repeat-reQuest (ARQ) is an error control technique widely used in digital data transmission. An ARQ system corrects erroneously received packets through retransmission of packets. The idea of using the ARQ strategy was first introduced by Chang [23], after which three classical ARQ schemes (or protocols) have been developed: stop-andwait (SW-ARQ), go-back-N (GBN-ARQ), and selective-repeat (SR-ARQ). These ARQ protocols were systematically articulated by Benice and Frey [15], and then thoroughly illustrated by Wicker [95].

In terms of the open system interconnection (OSI) reference model for layered net- 
work architectures [83], an ARQ protocol is usually located at the link layer (i.e., layer 2). Below and above it are the physical layer (or layer 1) and the network layer (or layer 3), respectively. From the ARQ's point of view, the physical layer provides forward channels (for data packets from the transmitter to the receiver) and feedback channels (for acknowledgement messages from the receiver to the transmitter), while the network layer provides/receives data packets. The transmitter sends packets to the receiver, which generates either a positive acknowledgement (ACK) or a negative acknowledgement (NACK) for each received packet based on the error detection result. Error detection is implemented by using a high-rate cyclic redundancy check (CRC) error-detection code [95].

In SW-ARQ, the transmitter sends a packet to the receiver and waits for its acknowledgement. Upon receiving a packet, the receiver generates either an NACK or an ACK for the received packet and sends it over a feedback channel. If an ACK is received, the transmitter sends out the next packet; otherwise, if an NACK is received, retransmission of the same packet will be scheduled immediately, and this process continues until the packet is positively acknowledged. In GBN-ARQ, the transmitter sends packets to the receiver continuously and receives acknowledgements as well. When an NACK is received, the transmitter retransmits the negatively acknowledged packet immediately and all following packets (positively or negatively acknowledged) already transmitted. In SR-ARQ, the transmitter sends packets continuously until an NACK arrives at the transmitter, in which case the transmitter retransmits the negatively acknowledged packet without re-sending the transmitted packets following it. To preserve the original arriving order of packets at the receiver in SR-ARQ, a buffer, referred to as the resequencing buffer, is provided at the receiver to store the correctly received packets that have not been released to the upper layer. 
An ARQ scheme plays such an important role in digital data transmission that study on ARQ continues to be a classical research area, which can be dated back to the 1960s. Many studies on ARQ reported in the last century have been based on the assumption that the error probability of packets does not change over time (i.e., the time-invariant or iid model of packet errors). This assumption is realistic for a satellite communication system, which was one of the originally proposed application areas of ARQ [58], and has been extensively used for modeling and analysis of ARQ protocols, such as work in $[6,15,20,29,31,32,51,62,63,71,72,77,78,80,81,84,90]$.

With the introduction of the third-generation wireless networks in the 1990s, data integrated with voice services have been provided over mobile wireless links. Since ARQ protocols achieve reliable transmission of packets over intrinsically unreliable wireless links, they have been used in the current and future generation wireless networks with the aim being the provisioning of multimedia services. In reality, the transmission condition of a wireless channel changes over time due to multi-path fading, scattering, and shadowing [64]. Consequently, a wireless channel is often severely affected by time-varying errors or losses of packets. That is, the probability that packets, when transmitted over a wireless channel, are erroneously received or simply lost, varies over time and these probabilities at different time instants may be correlated (i.e., the error burstiness). To reflect the Quality of Service (QoS) of a wireless system with ARQ, different packet error models (e.g., a finite state Markov chain model) have been employed for modeling and analysis of ARQ protocols in the past two decades or so, for instance, studies in $[7,11,12,13,30,36,49,52,60,61,65,73,74,75,89,92,99]$.

The above mentioned studies have shown that analysis simply based on an iid model of packet errors cannot give rise to a true QoS of a wireless data network with ARQ 
due to the non-realistic assumption. For instance, Lu and Chang [60] confirmed that the throughput performance of ARQ protocols for a Markov channel is better than that for a static channel. Kim and Krunz [49] showed that, when a Bernoulli packet arrival process is assumed, the mean transport delay (the duration from the time a packet arrives at the transmitter until its successful reception by the receiver) of SR-ARQ over a Markov channel is up to $100 \%$ longer than that of SR-ARQ over a static channel. Meanwhile, Rossi and Badia and Zorzi [74] reported that the mean packet delay (the duration from the time a packet is first time transmitted until its in-sequence delivery to the upper layer) of SRARQ over a static channel can be up to $100 \%$ longer than that of SR-ARQ over a Markov channel, when the transmitter is saturated with packets. All these results have shown that, an iid model, which neglects the time-varying property and the error burstiness in packet errors (and losses), is a misleading assumption for a time-varying channel in terms of the performance (e.g., throughput and delay) of ARQ protocols.

It is worthwhile to point out that, Rayleigh fading model [69], which describes how the signal propagates through the wireless medium, is a very popular channel model adapted in studies at the physical layer. Previous studies $[8,34,87,93,94,101]$ have used finitestate Markov chains to model Rayleigh fading channels. It is shown that a Markov process can be used to characterize the erroneous behavior of a Rayleigh fading channel and the accuracy of Markov models grows with the number of states used. This brings to researchers the theoretical basis through which relatively simple Markov chain models have been applied to the performance analysis of protocols in a higher layer, such as studies mentioned above in the link layer or $[5,7,88]$ in the TCP layer. This also motivates us to study SW-ARQ over multiple Markov channels. 


\subsection{Motivations}

In the series of next-generation wireless communication standards (e.g., IEEE 802.11 or WiFi [2], IEEE 802.16 or WiMax [3], high-speed Bluetooth [17], and UMTS [1]), orthogonal frequency division multiplexing (OFDM) $[22,14]$ and multiple-input multipleoutput antennas (MIMO) [96] are often adopted in a communication system to create multiple parallel channels, leading to a significantly increased data transmission rate in such a system. Unlike packet transmission over a single channel, in a multichannel communication system, multiple packets are sent simultaneously, i.e., one packet per channel, and packet transmission errors could occur across every channel. To implement error control through retransmission of packets in a multichannel communication system, ARQ protocols have been generalized to allow concurrent transmission of multiple packets $[70,91,102]$ and are becoming integral parts of wireless data services in High Speed Downlink Packet Access (HSDPA) [1, 33] and in WiMax [3, 39].

Among the three classical ARQ protocols (SW-ARQ, GBN-ARQ, SR-ARQ), SWARQ requires the least overhead and is the simplest one to implement. Since the transmitter remains idle during the round-trip propagation delay (RRT) for the packet, SW-ARQ is more suitable for short-distance communication systems. In real-life implementations, SW-ARQ has been standardized in the specification of Bluetooth [18] for single-channel communications, and is expected to be part of the standard for high-speed Bluetooth technology [17] in a multichannel communication environment. Another example is the adoption of SW-ARQ over multiple channels in HSDPA [1, 33] to achieve a compatible system throughput performance with SR-ARQ [91]. A communication system using SW-ARQ over multiple channels is also proposed in patent [40]. 
Unlike studies on ARQ protocols for single-channel communications, only a limited number of publications are available on multichannel ARQ protocols. Chang and Yang [21] studied performance of the three classical ARQ protocols for multiple identical channels (i.e., all channels have the same transmission rate and the same time-invariant error rate). In that study, expressions for the throughput, which is the average number of packets successfully transmitted per unit of time, and the mean transmission delay, which is the average time between the instant at which a packet is transmitted for the first time and the instant at which it is successfully received, have been derived. Meanwhile, Wu and Vassiliadis and Chung [98] conducted a throughput performance study on multichannel ARQ protocols based on the same model as that in [21]. The resequencing issue in multichannel ARQ protocols was first addressed by Shacham and Chin [79]. In that study, they conducted a resequencing analysis (e.g., the resequencing buffer occupancy and the resequencing delay) for SR-ARQ over parallel channels, all of which have the same transmission rate but possibly different time-invariant error rates. The authors in [35] analyzed the packet delay distribution function of GBN-ARQ for a generic number of parallel channels, each of which has the same transmission rate but a possibly different fixed error rate. Recently, Ding and Rice [25, 27] considered ARQ protocols for parallel channels, each having unique transmission rate and error rate. An optimal packet scheduling rule has been identified and an expression for system throughput has been derived in [25, 27]. $\mathrm{Hu}$ and Afshartous [44] analyzed the throughput performance of SW-ARQ for multiple channels in HSDPA using simulation. Ding [26] derived an approximate expression of the mean packet delay for multichannel ARQ protocols. Sima and Zhou and Zhang [82] evaluated the system throughput performance of SR-ARQ for multiple time-varying channels through simulation analysis. 
From the above discussion, it is clear that SW-ARQ for multiple channels has important real-world applications, but exact analysis of multichannel SW-ARQ models is very limited, which motivated the research in this thesis in one part. Moreover, all above mentioned studies on multichannel ARQ protocols have been based on the assumption of a time-invariant error rate for each channel except [82], in which only simulation analysis has been conducted. Similar to studies in the context of single-channel communications, we expect that these results based on time-invariant error rates of channels may not reflect the true QoS of a communication system containing multiple wireless channels characterized by time-varying packet errors. There has been no study reported in the literature for a systematical analysis of a multichannel ARQ model characterizing time-varying packet errors, although studies (see Section 1.1) on ARQ for a single channel of time-varying models have been extensively conducted. This motivated another part of the thesis research.

Through the thesis research, we not only extend early studies on ARQ protocols for multiple channels with time-invariant error rates, but also generalize the results in published works on ARQ protocols for single time-varying channels (e.g., Markov channels).

It is worthwhile to point out that hybrid-ARQ, in which an erroneously received packet is corrected by a combination of ARQ and forward-error-correction (FEC) [56, 97], for multiple time-varying channels, has also received an increasing research attention recently, for instance, studies in $[24,37,38,50,66,67,68,76,85,86]$. While having the impetus to improve the throughput performance of fading MIMO systems, these studies have been conducted from the coding perspective at the physical layer, which is not within the scope of this thesis research. 


\subsection{Contributions}

Several contributions presented in this thesis are listed below.

\section{- Development of stochastic systems for multichannel SW-ARQ}

Two stochastic systems of SW-ARQ for multiple channels are developed for performance evaluation. These stochastic systems are not only adapted to real-world implementations, but also mathematically tractable. This leads to a systematic method proposed in this thesis for tackling problems of more complex ARQ schemes over multiple Markov channels.

- Expression of the probability generating function of the resequencing buffer occupancy

The probability generating function of the resequencing buffer occupancy for MSWARQ-inS over both iid and Markov channels is obtained. As shown in examples, the distribution function of the resequencing buffer occupancy can be efficiently computed based on the probability generating function. To the best of our knowledge, this is the first research to report analytical results of the resequencing buffer for an ARQ scheme over multiple time-varying channels (i.e., Markov channels).

\section{- Expression of the probability mass function of the resequencing delay}

The probability mass function of the resequencing delay for MSW-ARQ-inS over multiple iid channels is obtained. The function is numerically computed through examples, from which it is observed that the probability that a packet goes through the resequencing buffer without delay decreases with the increase of either the number of channels or the average error rate of the channels. 


\section{- Development of a general method for analysis of the packet delay}

Through the analysis of the packet delay for the models, a general method used for analyzing the distribution function of the packet delay is established. Instead of using the standard method in which the stationary distribution of a constructed Markov chain is used to express the system performance metrics, we derive the distribution function through the absorption analysis of a transient Markov chain in this research.

\section{- Identification of the impact on the model performance}

Through numerical and simulation analysis, we investigate the impact of various protocol parameters on the system performance. The mean resequencing buffer occupancy and the mean resequencing delay for MSW-ARQ-inS grow with the increase of either the number of channels or the average error rate of channels, but decrease as the variance in the error rates increases. For both MSW-ARQ and MSW-ARQ-inS, the mean packet delay increases with the average error rate but decreases with the variance in the error rates, while the number of parallel channels has only an insignificant impact on the mean packet delay. Moreover, with the dynamic scheduling, MSW-ARQ-inS always outperforms MSW-ARQ in terms of the mean packet delay performance. We also confirm the result that the dynamic packet scheduling always outperforms the static scheduling from the mean packet delay performance criterion, a result which has been obtained in previous studies based on the throughput criterion. 


\subsection{Thesis Outline}

The thesis is organized as follows. A communication system containing multiple parallel channels is described in Chapter 2. Two stochastic system, MSW-ARQ and MSW-ARQinS, for modeling multichannel SW-ARQ, are introduced in Chapter 3. Resequencing analysis of the MSW-ARQ-inS model is performed in Chapter 4, and packet delay is analyzed for both MSW-ARQ and MSW-ARQ-inS in Chapter 5. Concluding remarks are made and possible future work is suggested in Chapter 6. 


\section{Chapter 2}

\section{Multichannel Systems with ARQ}

In this chapter, a communication system containing multiple parallel channels is described and some assumptions on the system are stated in Section 2.1. Two channel models used in this study are defined in Section 2.2. Multichannel SW-ARQ models, which will be analyzed in the following chapters, are based on the system and the assumptions discussed in this chapter unless specified otherwise.

\subsection{A Multichannel System with ARQ Error Control}

A multichannel data communication system, in which a transmitter-receiver pair communicates data packets for one communication (e.g., a large-size video file transfer), is illustrated in Fig. 2.1. The communication link connecting the transmitter and the receiver consists of $M(M \geq 2)$ parallel channels numbered from 1 to $M$, each of which is characterized by a data transmission rate and a channel model. The transmission rate of a channel is measured by the maximum number of packets that can be transmitted over that 
channel during a specified time period, while the channel model, or the model of packet errors, describes the statistical property of transmission errors of packets when they are transmitted over the channel. In this study, two channel models (to be discussed in Section 2.2) will be considered with the assumption that packet errors occurring in different channels are mutually independent. A feedback channel is also provided in the system. We assume that an erroneous packet can always be detected through CRC coding and that the feedback channel is error-free for transmitting acknowledgement packets.

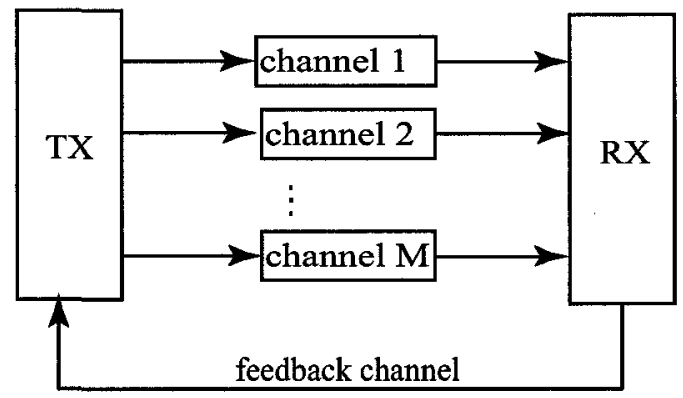

TX and RX denote transmitter and receiver, respectively.

Figure 2.1: A Multichannel System with ARQ

Each packet to be transmitted is identified by a unique integer number, referred to as the sequence number. We assume that there is a buffer in the transmitter, referred to as the transmission queue, in which infinitely many packets are waiting according to their sequence numbers for first-in-first-out transmission and retransmission. That is, there are always packets in the system to be transmitted, which in related studies is referred to as the heavy traffic condition. We also assume that all channels have the same transmission rate, and that the $M$ channels are time-slotted with one unit (or slot) equal to the transmission time of a packet over a channel. Therefore, the transmission rate of each channel is one packet per slot. All packets, when transmitted from the transmitter to the receiver, have 
a fixed round trip time (RRT) equal to $(m-1)$ slots, which is assumed to be an even number of slots. (Therefore, $m$ slots represent the sum of the transmission time and RTT of a packet.) A packet experiences the same propagation delay in forward and feedback channels, which is $(m-1) / 2$ slots. Once packet transmission starts, the transmitter sends multiple packets at a time, one per channel. All channels share the same set of sequence numbers of the packets in packet-to-channel scheduling. A multichannel ARQ model, which is to be described in the next chapter, is used in the system for packet error control.

\subsection{Channel Models}

A channel model, or a model of packet errors, defines the statistical property of transmission errors when packets are transmitted over a physical channel. We consider two models of packet errors, iid model and Markov model, for this study.

\subsubsection{The iid Model}

In the case of an iid model, the transmission error of a packet when it is transmitted over the channel is characterized by a time-invariant error rate representing the probability that the packet is erroneously received or simply lost.

\subsubsection{The Markov Model}

In the Markov model, the packet error property over the Markov channel is characterized by the Gilbert-Elliott model $[28,41]$, in which the state of the channel is modeled as a two-state Markov chain $\{E(t): t=0,1, \cdots\}$. The state space of the Markov chain 
$\{E(t): t=0,1, \cdots\}$ is

$$
\{G O O D, B A D\}
$$

and the transition matrix of the Markov chain $\{E(t): t \geq 0\}$ is

$$
\left[\begin{array}{cc}
1-\alpha & \alpha \\
\beta & 1-\beta
\end{array}\right] .
$$

The packet error rate when the channel is in "GOOD" is $e_{G}$, and the packet error rate corresponding to " $B A D$ " is $e_{B}$. We let $0 \leq e_{G}<e_{B} \leq 1$, since the case where $e_{G}=e_{B}$ corresponds to an iid model with the error rate $e_{G}$.

The Markov chain $\{E(t): t \geq 0\}$ has the stationary distribution given by

$$
\left(\frac{\alpha}{\alpha+\beta}, \frac{\beta}{\alpha+\beta}\right)
$$

For the Gilbert-Elliott model, we define the following parameters

$$
\sigma=\frac{\alpha}{\alpha+\beta} \quad \text { and } \quad \chi=\frac{1}{\alpha+\beta} .
$$

The two pairs of parameters $(\chi, \sigma)$ and $(\alpha, \beta)$ are uniquely determined by each other. In steady state, the stationary distribution of the error process $\{E(t): t \geq 0\}$ can be re-written as

$$
(\sigma, 1-\sigma)
$$

\subsection{Summary}

In this chapter, we described a multichannel communication system and the assumptions on which our analysis of multichannel ARQ models is based. We defined two channel models, which will be used for this research. 


\section{Chapter 3}

\section{Multichannel SW-ARQ Models}

In this chapter, we introduce two multichannel SW-ARQ systems in Section 3.1, and define performance metrics used for evaluating these models in Section 3.2.

\subsection{Multichannel SW-ARQ Models}

In this section, we describe two stochastic systems for modeling multichannel SW-ARQ, which are MSW-ARQ and MSW-ARQ-inS. The following error control procedure is valid for both models, while different actions are discussed in Section 3.1.1 for MSW-ARQ and in Section 3.1.2 for MSW-ARQ-inS.

1. At the beginning of a slot $t m, t=0,1, \cdots$ (see Fig. 3.1), the transmitter starts transmitting a block of $M$ packets to the receiver, and completes transmission at the end of the slot. Before the transmitter sends the next block of $M$ packets in slot $(t+1) m$, it is idle.

2. The receiver receives the block of $M$ packets at the end of slot $t m+(m-1) / 2$. The 
packet transmitted over channel $i$ is received erroneously or simply lost according to the packet error model corresponding to channel $i$. Which packet error model for the channel is used will be specified when analysis is performed in the following chapters. After the error detection, the receiver sends an acknowledgement packet, which contains exactly $M$ acknowledgements (ACKs/NACKs) corresponding to the most recently received block of $M$ packets, to the transmitter. We assume that error detection and transmission of acknowledgement packets take no time at the receiver, so that they are both completed at the end of slot $t m+(m-1) / 2$.

3. After sending the acknowledgement packet, the receiver takes different actions on the $M$ packets, a process which will be discussed in Section 3.1.1 for MSW-ARQ and in Section 3.1.2 for MSW-ARQ-inS. The transmitter receives the acknowledgement packet at the end of slot $(t m+m-1)$. It checks each acknowledgement in the acknowledgement packet, and prepares the next block of $M$ packets to transmit according to rules to be stated in Section 3.1.1 for MSW-ARQ and in Section 3.1.2 for MSW-ARQ-inS.

4. To transmit the next block of $M$ packets in slot $(t+1) m$, a packet-to-channel scheduling policy needs to be specified. There are two packet scheduling policies, the static scheduling and the dynamic scheduling, to be considered in this study. For the static scheduling, an old packet (i.e., a packet to be retransmitted) is always retransmitted over the same channel as the originally assigned one, and new packets selected to be transmitted are randomly assigned to the channels available for transmitting new packets. With the dynamic scheduling, the transmitter assigns the packet with the smallest sequence number (either an old unqualified or a new 
packet) in the block to channel 1 , the packet with the second smallest sequence number to channel 2 , and so forth.

5. We assume that the transmitter completes acknowledgement checking and preparation of the next block to transmit instantaneously. Then, the transmitter starts transmitting the next block of $M$ packets at the beginning of slot $(t+1) m$ and completes transmission at the end of the slot.

As described above, the different actions between MSW-ARQ and MSW-ARQ-inS are those in item 3, which are discussed in detail below.

\subsubsection{MSW-ARQ}

A qualified packet is a correctly received packet with a sequence number such that all packets with a smaller sequence number have been correctly received. After sending the acknowledgement packet at the end of slot $(t m+(m-1) / 2)$, the receiver delivers all qualified packets to the upper layer and discards all unqualified packets received. The transmitter prepares the next block of $M$ packets to transmit in slot $(t+1) m$ according to the following rule: if no NACK is contained in the acknowledgement packet, the next block to transmit is composed of $M$ (new) packets that will be transmitted for the first time; if the acknowledgement packet contains one or more NACKs, however, the next block of $M$ packets contain those (old) unqualified and possibly new packets. MSW-ARQ with the dynamic scheduling for three time-invariant channels (discussed in Section 4.1) with $p_{1} \leq p_{2} \leq p_{3}$, where $p_{i} \in(0,1)$ represents the error rate for channel $i$, is illustrated in Fig. 3.1. 

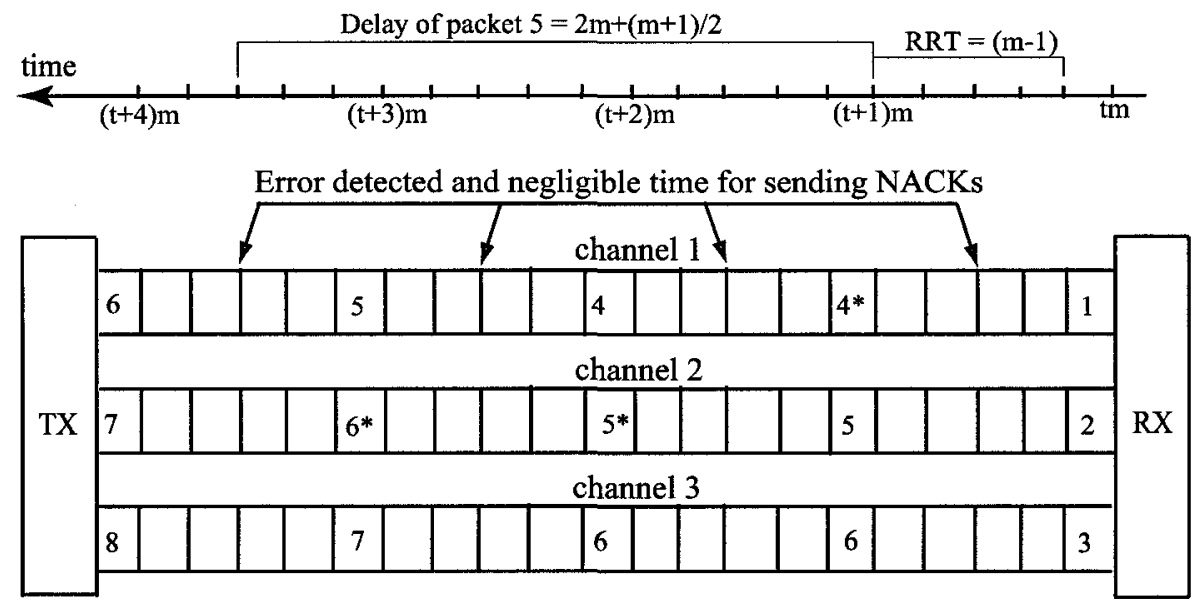

*, TX, RX denote transmission error, transmitter, receiver, respectively. Integer numbers in boxes are sequence numbers of packets.

Figure 3.1: $M S W-A R Q(M=3, m=5)$

\subsubsection{MSW-ARQ-inS}

In MSW-ARQ-inS, which is a variant of MSW-ARQ, the transmitter retransmits only erroneously received packets. In order to achieve packets' delivery in the order of their sequence numbers (i.e., in-sequence delivery), a resequencing buffer is provided at the receiver to temporarily store undeliverable packets. An undeliverable packet is a correctly received but unqualified packet (i.e., there is another packet with a smaller sequence number, which has not been received correctly so far). After sending the acknowledgement packet at the end of slot $(t m+(m-1) / 2)$, the receiver discards erroneously received packets and stores correctly received ones in the resequencing buffer. Then, it delivers all qualified packets, as defined in Section 3.1.1, from the resequencing buffer to the upper layer, and continues storing undeliverable packets for future delivery. The transmitter prepares the next block of $M$ packets to transmit in slot $(t+1) m$ according to the following rule: if there is no NACK in the acknowledgement packet, the next block to transmit is 
composed of $M$ new packets; if the acknowledgement packet contains one or more, for example $k$, NACKs, the next block on $M$ packets consist of those $k$ old packets, which are negatively acknowledged by the receiver, and $M-k$ new packets. An example of MSWARQ-inS with the dynamic scheduling for three time-invariant channels (discussed in Section 4.1) with $p_{1} \leq p_{2} \leq p_{3}$, where $p_{i} \in(0,1)$ represents the error rate for channel $i$, is illustrated in Fig. 3.2.
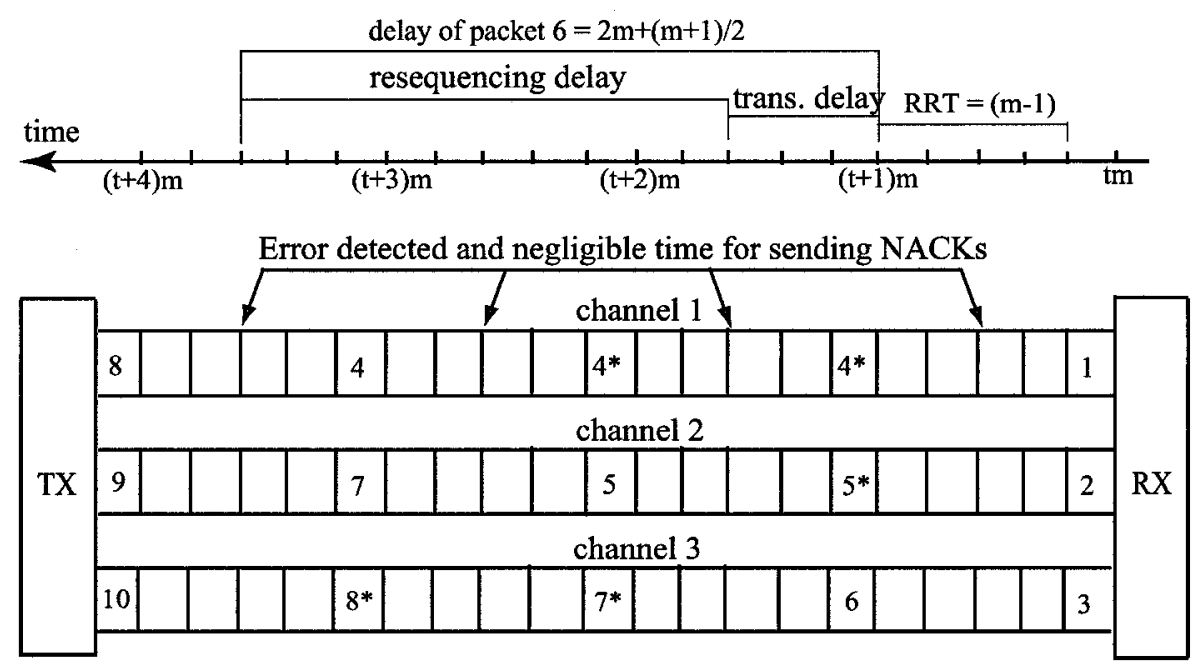

*, TX, RX denote transmission error, transmitter, receiver, respectively. Integer numbers in boxes are sequence numbers of packets.

Figure 3.2: MSW-ARQ-inS (M=3, $m=5)$

\subsection{Study Objective and Performance Metrics}

With the settings described above, both MSW-ARQ and MSW-ARQ-inS implement error control while packets are delivered to the upper layer in the order of their sequence numbers (or in-sequence delivery). However, the performance, such as throughput and delay, of one model is different from the other model, and thus it needs to be investi- 
gated under various channel conditions. The main purpose of this study is to carry out steady-state analysis of the two multichannel SW-ARQ models when they are used for error control. We establish a new method used to evaluate the packet delay performance of the multichannel SW-ARQ models, conduct a resequencing analysis for MSW-ARQ-inS, and identify the impact of packet scheduling policies on the model performance through numerical and simulation analysis.

In our steady-state analysis of the MSW-ARQ model, the performance metric is the packet delay. The delay of a packet is defined as the amount of time between the instant at which the packet is transmitted for the first time and the instant at which it is delivered to the upper layer (see Fig. 3.1 for illustration of the delay of packet with sequence number $5)$.

In the performance analysis of the MSW-ARQ-inS model, in addition to the analysis of the packet delay for the model, analysis of the resequencing buffer is also conducted. The resequencing buffer is analyzed through deriving statistical properties of resequencing buffer occupancy and resequencing delay in the equilibrium regime. The resequencing buffer occupancy is the number of packets waiting in the resequencing buffer for delivery. The resequencing delay of a packet is defined as the amount of waiting time of the packet in the resequencing buffer. In MSW-ARQ-inS, we use transmission delay to denote the time period from the instant at which a packet is transmitted for the first time until its correct receipt by the receiver. Then the packet delay is the sum of the transmission delay and the resequencing delay. (See Fig. 3.2 for illustration of the resequencing delay and the transmission delay of packet with sequence number 6.) 


\subsection{Summary}

In this chapter, we discussed two multichannel SW-ARQ models, stated the purpose of this research, and defined the performance measures to be used for analyzing the models. 


\section{Chapter 4}

\section{Resequencing Analysis of}

\section{MSW-ARQ-inS}

In this chapter, we investigate the queueing behavior of packets in the resequencing buffer for MSW-ARQ-inS over time-invariant channels and Markov channels in Section 4.1 and Section 4.2, respectively. Based on the analytical results, we perform numerical and simulation analysis in Section 4.3, followed by concluding remarks of this chapter made in Section 4.4. The results presented in this chapter are to be published in [53].

When the resequencing buffer is provided at the receiver, the queueing behavior of packets in the resequencing buffer plays an important role in the overall protocol performance. Therefore, many studies have focused on analysis of the resequencing buffer in the context of either SR-ARQ or a general queueing network setting. The reader is referred to Appendix A for a brief literature survey on packet resequencing studies. 


\subsection{Analysis for Time-invariant Channels}

In this section, we investigate statistical properties of the resequencing buffer occupancy and the resequencing delay when the time-invariant error model (i.e., the iid model) of channels is assumed. That is, the error rate for channel $i$, for $i=1, \cdots, M$, is $p_{i} \in(0,1)$, and $p_{i}$ might be different from $p_{j}$ for $i \neq j$.

Without loss of generality, the $M$ channels are numbered from 1 to $M$ such that $p_{1} \leq$ $p_{2} \leq \cdots \leq p_{M}$. The dynamic scheduling is implemented as follows. To send the next block of $M$ packets in slot $(t+1) m$, the transmitter assigns the packet with the smallest sequence number in the block to channel 1 , then the packet with the second smallest sequence number to channel 2 , and so forth.

We denote by $C$ an arbitrary packet of our interest and let slot 0 represent the slot at the end of which $C$ is received for the first time. Given that $C$ is received for the first time over channel $i$, for $i=1, \cdots, M$, we define the following two random variables for each $t=0,1, \cdots$.

1. $X_{i}(t)$ is the number of packets, in the block of $M$ packets received at the end of slot $t m$, whose sequence numbers are not larger than that associated with $C$.

2. $Y_{i}(t)$ is the number of packets, in the block of $M$ packets received at the end of slot $t m$, whose sequence numbers are not larger than that associated with $C$, if $C$ is contained in the block, and zero otherwise.

The stochastic processes $\left\{X_{2}(t): t=0,1, \cdots\right\}$ and $\left\{Y_{2}(t): t=0,1, \cdots\right\}$ are illustrated in Figure 4.1. Moreover, both process $\left\{X_{M}(t): t=0,1, \cdots\right\}$ and process 

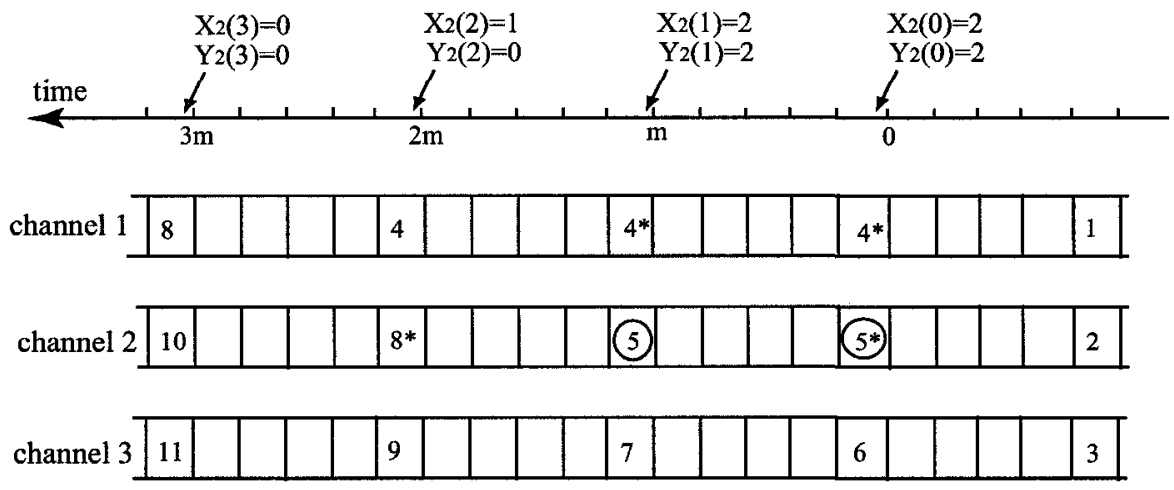

* denotes transmission error, (5) denotes the packet of our interest.

Figure 4.1: Processes $\left\{X_{i}(t): t \geq 0\right\}$ and $\left\{Y_{i}(t): t \geq 0\right\}(\mathrm{M}=3, \mathrm{~m}=5)$

$\left\{Y_{M}(t): t=0,1, \cdots\right\}$ are Markov chains with the same state space

$$
\{0,1, \cdots, M\}
$$

We define

$$
q_{k}=1-p_{k}, k=1, \cdots, M
$$

and

$$
\Omega_{i}=\{1, \cdots, i\}
$$

Then we let $B_{i, j}$ denote a subset of $\Omega_{i}$ of size $j$, and $B_{i, j}^{c}=\Omega_{i} \backslash B_{i, j}$. The product over an empty set $\Phi$ is defined to be one, i.e.,

$$
\prod_{k \in \Phi} q_{k}=\prod_{k \in \Phi} p_{k}=1
$$


The transition matrix of the Markov chain $\left\{X_{M}(t): t=0,1, \cdots\right\}$ is given by

$$
\mathbf{P}=\left[\begin{array}{lllll}
1 & 0 & 0 & \cdots & 0 \\
P_{10} & P_{11} & 0 & \cdots & 0 \\
P_{20} & P_{21} & P_{22} & \cdots & 0 \\
\vdots & \vdots & \vdots & \ddots & \vdots \\
P_{M, 0} & P_{M, 1} & P_{M, 2} & \cdots & P_{M, M}
\end{array}\right]
$$

where

$$
P_{i j}=\sum_{B_{i, j} \subseteq \Omega_{i}}\left(\prod_{k \in B_{i, j}} p_{k} \prod_{k \in B_{i, j}^{c}} q_{k}\right),
$$

for $i=1, \cdots, M$ and $j=0, \cdots, i$. The transition matrix of the Markov chain $\left\{Y_{M}(t)\right.$ : $t=0,1, \cdots\}$ is given by

$$
\mathbf{Q}=\left[\begin{array}{l|l}
1 & \mathbf{0} \\
\hline \mathbf{q} & \mathbf{T}
\end{array}\right]=\left[\begin{array}{l|llll}
1 & 0 & 0 & \cdots & 0 \\
\hline q_{1} & p_{1} & 0 & \cdots & 0 \\
q_{2} & Q_{21} & Q_{22} & \cdots & 0 \\
\vdots & \vdots & \vdots & \ddots & \vdots \\
q_{M} & Q_{M 1} & Q_{M 2} & \cdots & Q_{M M}
\end{array}\right]
$$

where,

$$
Q_{i j}=p_{i} P_{i-1, j-1}
$$

for $i=2, \cdots, M$ and $j=1, \cdots, i$, where $P_{i-1, j-1}$ is given in (4.5).

We note that the $(i+1) \times(i+1)$ upper-left submatrix of $\mathbf{P}$, which is lower triangular, is the transition matrix of the Markov chain $\left\{X_{i}(t): t \geq 0\right\}$. This implies that for $j, l$ in the state space of the Markov chain $\left\{X_{i}(t): t \geq 0\right\}$,

$$
\mathbb{P}\left[X_{i}(t+n)=l \mid X_{i}(t)=j\right]=\mathbb{P}\left[X_{M}(t+n)=l \mid X_{M}(t)=j\right]=P_{j l}^{(n)},
$$


where $P_{j l}^{(n)}$ is the $n^{\text {th }}$ step transition probability from $j$ to $l$ of the Markov chain $\left\{X_{M}(t)\right.$ : $t \geq 0\}$. This is also true for the Markov chain $\left\{Y_{M}(t): t \geq 0\right\}$.

As illustrated below, the constructed Markov chains are used to analyze the resequencing buffer occupancy and the resequencing delay in steady state.

\subsubsection{Resequencing Buffer Occupancy}

We let $B_{r}$ represent the resequencing buffer occupancy at the beginning of a slot, referred to as the observation instant. At the end of the slot, a block of $M$ packets are received. The probability generating function $\mathcal{G}_{B_{r}}(z)$ of $B_{r}$ is given in the following theorem.

\section{Theorem 4.1.}

$$
\begin{aligned}
\mathcal{G}_{B_{r}}(z)=P_{M 0}+ & \sum_{i=1}^{M} \sum_{n=1}^{\infty} \sum_{x_{1}=0}^{i-1} \sum_{x_{2}=0}^{x_{1}} \cdots \sum_{x_{n-1}=0}^{x_{n-2}} \\
& \left(\prod_{j=1}^{M}\left(p_{j}+q_{j} z\right)^{\mu_{j}}\right) \mathbb{P}\left[C\left(n, i, x_{1}, \cdots, x_{n-1}\right)\right],
\end{aligned}
$$

where,

$$
\mathbb{P}\left[C\left(n, i, x_{1}, \cdots, x_{n-1}\right)\right]=\sum_{\delta=0}^{i-1} P_{M \delta}\left(P_{i-1, x_{1}} P_{x_{1}, x_{2}} \cdots P_{x_{n-1}, 0} p_{i} \prod_{k=1}^{n-1} p_{x_{k}+1}\right)
$$

and

$$
\mu_{j}= \begin{cases}n-1-\max \left\{k: 0 \leq k \leq n-1, x_{k}+1 \geq j\right\}, & \text { if } j \leq i, \\ n, & \text { otherwise. }\end{cases}
$$

Proof : The packet received over channel 1 at the end of the slot, at which the resequencing buffer is observed, is denoted by $C_{o}$. Due to the dynamic scheduling, $C_{o}$ has the smallest sequence number among all packets that have not been correctly received so far. 
Therefore, only those correctly received packets with a larger sequence number than that associated with $C_{o}$ will contribute to $B_{r}$. We define $A_{n}$ to be the event that $C_{o}$ has been (incorrectly) received for $n(n=0,1, \cdots)$ times until the observation instant. If $A_{0}$ is true, the oldest packet $C_{o}$ has never been received before, and therefore, the resequencing buffer is empty at the observation instant. When $A_{n}$ is true for $n \geq 1$, we let $A_{n, i}$ be the sub-event that $C_{o}$ is received for the first time over channel $i$, for $i=1, \cdots, M$, and has been (incorrectly) received for $n$ times. Now we denote by $t m$ the slot at the end of which $C_{o}$ is received for $(t+1)^{s t}$ time. When the event $A_{n, i}$ is true, we have the process $\left\{X_{M}(t): t=0, \cdots, n\right\}$ by considering the packet received over channel $i-1$ in slot 0 as the packet of our interest (see Figure 4.2). Moreover,

$$
X_{M}(0)=i-1
$$

and

$$
X_{M}(n)=0
$$

By conditioning on a sample path of the Markov chain $\left\{X_{M}(t): t=0, \cdots, n\right\}$, we have

$$
\mathbb{P}\left[A_{n, i}\right]=\sum_{x_{1}=0}^{i-1} \sum_{x_{2}=0}^{x_{1}} \cdots \sum_{x_{n-1}=0}^{x_{n-2}} \mathbb{P}\left[C\left(n, i, x_{1}, \cdots, x_{n-1}\right)\right]
$$

where the event $C\left(n, i, x_{1}, \cdots, x_{n-1}\right)$ is the joint event of the following three independent events.

- At most $i-1$ packets in the $M$ packets received in slot $-m$ (i.e., $m$ slot before slot 0 ) are packets retransmitted in slot 0 , which has the probability $\sum_{\delta=0}^{i-1} P_{M \delta}$.

- The path of the Markov chain $\left\{X_{M}(t): t=0, \cdots, n\right\}$ from step 0 to step $n$ is $\left\{X(0)=i-1, X(1)=x_{1}, \cdots, X(n-1)=x_{n-1}, X(n)=0\right\}$. 
- $C_{o}$ is received erroneously in slots $0, m, \cdots,(n-1) m$, whose probability is given by $p_{i} \prod_{k=1}^{n-1} p_{x_{k}+1}$.

Therefore, the probability $\mathbb{P}\left[C\left(n, i, x_{1}, \cdots, x_{n-1}\right)\right]$ is given in (4.10).

When the joint event $C\left(n, i, x_{1}, \cdots, x_{n-1}\right)$ occurs, the packets that are received correctly between slot 0 and slot $(n-1) m$ and have a larger sequence number than that associated with $C_{o}$ contribute to the resequencing buffer occupancy $B_{r}$. For each channel $j=1, \cdots, M$, the number of slots in which packets with a larger sequence number are received (either erroneously or correctly) between slot 0 and slot $(n-1) m$ is

$$
\mu_{j}= \begin{cases}n-1-\max \left\{k: 0 \leq k \leq n-1, x_{k}+1 \geq j\right\}, & \text { if } j \leq i \\ n, & \text { otherwise }\end{cases}
$$

An example of $\mu_{j}$ is shown in Figure 4.2.

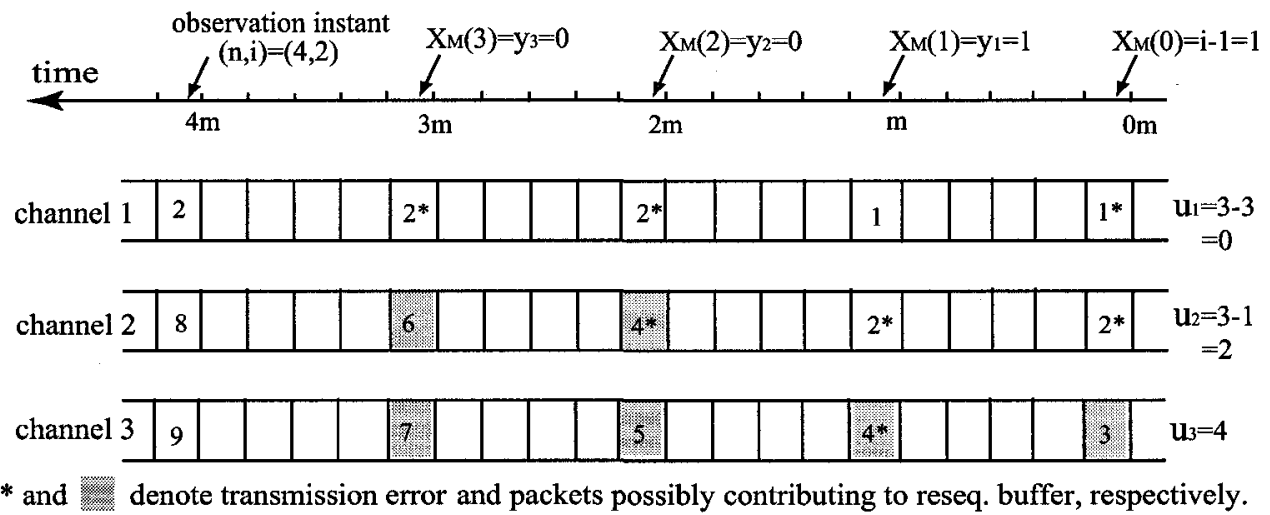

Figure 4.2: Example of $\mu_{j}(\mathrm{M}=3, \mathrm{~m}=5)$

If we let $B_{r}^{j}$ represent the number of packets that contribute to $B_{r}$ over channel $j$, it is a Binomial random variable with parameters $\mu_{j}$ and $q_{j}$. According to the assumption of mutual independence between different channels, $B_{r}^{1}, \cdots, B_{r}^{M}$ are independent. 
Therefore, when $A_{n, i}$ is true, the conditional pgf of $B_{r}$ is

$$
\begin{aligned}
& \mathcal{G}_{B_{r} \mid A_{n, i}}(z \mid n, i) \\
= & \sum_{x_{1}=0}^{i-1} \sum_{x_{2}=0}^{x_{1}} \cdots \sum_{x_{n-1}=0}^{x_{n-2}} \mathbb{E}\left[z^{\sum_{j=1}^{M} B_{r}^{j}} \mid A_{n, i}, X_{M}(k)=x_{k}, 1 \leq k \leq n-1\right] \times \\
& \mathbb{P}\left[X_{M}(k)=x_{k}, 1 \leq k \leq n-1 \mid A_{n, i}\right] \\
= & \frac{1}{\mathbb{P}\left[A_{n, i}\right]} \sum_{x_{1}=0}^{i-1} \sum_{x_{2}=0}^{x_{1}} \cdots \sum_{x_{n-1}=0}^{x_{n-2}}\left(\prod_{j=1}^{M}\left(p_{j}+q_{j} z\right)^{\mu_{j}}\right) \mathbb{P}\left[C\left(n, i, x_{1}, \cdots, x_{n-1}\right)\right]
\end{aligned}
$$

where the last equality is due to the fact that a Binomial random variable $B_{N, 1-p}$ (with parameters $N$ and $1-p$ ) has the pgf

$$
\mathcal{G}_{B_{N, 1-p}}(z)=(p+z(1-p))^{N}
$$

Since $A_{0}$ is true if and only if all $M$ packets received in slot $-m$ ( $m$ slots before slot $0)$ are received successfully,

$$
\mathbb{P}\left[A_{0}\right]=\mathbb{P}\left[X_{M}(0)=0 \mid X_{M}(-1)=M\right]=P_{M 0}
$$

By conditioning on the events $A_{0}$ and $A_{n, i}$, the pgf $\mathcal{G}_{B_{r}}$ of $B_{r}$ is obtained and given in (4.9). Theorem 4.1 is proved.

\subsubsection{Resequencing Delay}

With the construction of Markov chain $\left\{X_{M}(t): t \geq 0\right\}$, the probability that $C$ is transmitted for the first time over channel $i$, for $i=1, \cdots, M$, is given in the following lemma.

Lemma 4.2. For each $i=1,2, \cdots, M$, we denote by $\Lambda_{i}$ the event that $C$ is transmitted for the first time over channel $i$. Then,

$$
\mathbb{P}\left[\Lambda_{i}\right]=\frac{1}{1-P_{M M}} \sum_{\delta=(M+1)-i}^{M} \frac{P_{M, M-\delta}}{\delta} .
$$


Proof : If $\Lambda_{i}$ occurs, in the block of $M$ packets received in slot $-m$ ( $m$ slots before slot 0 ), there is at least one packet that is correctly received. We let $W$ be the number of packets received correctly in slot $-m$. In other words, $W$ is the number of packets that are received for the first time in slot 0 . By the definition of $X_{M}(t)$, we have

$$
\begin{aligned}
\mathbb{P}[W=\delta] & =\frac{\mathbb{P}\left[X_{M}(0)=M-\delta \mid X_{M}(-1)=M\right)}{\sum_{k=1}^{M} \mathbb{P}\left[X_{M}(0)=M-k \mid X_{M}(-1)=M\right)} \\
& =\frac{P_{M, M-\delta}}{1-P_{M M}}, \delta=1, \cdots, M
\end{aligned}
$$

If $W=\delta$, the packets received over channels $(M-\delta+1), \cdots, M$ are new packets (received for the first time) in slot 0 . Since $C$ is an arbitrarily chosen packet that must be received for the first time in slot 0 ,

$$
\mathbb{P}\left[A_{i} \mid W=\delta\right]=\left\{\begin{array}{l}
\frac{1}{\delta}, \text { if } i=(M-\delta+1), \cdots, M \\
0, \text { otherwise }
\end{array}\right.
$$

By conditioning on the event $\{W=\delta\}$, we have the probability of $A_{i}$ as given in (4.19). Lemma 4.2 is proved.

Clearly, the Markov chain $\left\{Y_{M}(t): t \geq 0\right\}$ is transient with the absorbing state 0 . If $\mathbf{S}$ denotes the fundamental matrix [19] of the Markov chain $\left\{Y_{M}(t): t \geq 0\right\}$, then, we have

$$
\mathbf{S} \equiv \sum_{n=0}^{\infty} \mathbf{T}^{n}=(\mathbf{I}-\mathbf{T})^{-1}
$$

We denote by $D_{r}$ the resequencing delay of the packet $C$. Then the distribution function of $D_{r}$ is given in the following theorem.

Theorem 4.3. In steady state, if

$$
d_{r}(x) \equiv \mathbb{P}\left[D_{r}=x m\right], x=0,1, \cdots,
$$


then,

$$
d_{r}(x)= \begin{cases}\sum_{i=1}^{M} \mathbb{P}\left[\Lambda_{i}\right] \sum_{k=1}^{i} P_{k-1,0} q_{k} S_{i k}, & \text { if } x=0 \\ \sum_{i=2}^{M} \mathbb{P}\left[\Lambda_{i}\right] \sum_{k=2}^{i} q_{k} S_{i k}\left(\sum_{j=1}^{k-1} P_{k-1, j}^{(x)} P_{j 0}\right), & \text { if } x \geq 1\end{cases}
$$

where $\mathbb{P}\left[\Lambda_{i}\right]$ is given by (4.19).

Proof : If $\Lambda_{i}$ occurs, we denote by $\Gamma_{n, k}$ the event that $C$ is received correctly over channel $k$ for the $n^{t h}$ transmission with $n=1,2, \cdots$ and $k=1, \cdots, i$. By the definition of the process $\left\{Y_{i}(t): t \geq 0\right\}$, we have

$$
\begin{aligned}
\mathbb{P}\left[\Gamma_{1, k} \mid \Lambda_{i}\right] & =\mathbb{P}\left[Y_{i}(1)=0, Y_{i}(0)=k \mid Y_{i}(0)=i\right] \\
& = \begin{cases}q_{i}, & \text { if } k=i \\
0, & \text { otherwise }\end{cases}
\end{aligned}
$$

and for $n \geq 2$,

$$
\begin{aligned}
\mathbb{P}\left[\Gamma_{n, k} \mid \Lambda_{i}\right] & =\mathbb{P}\left[Y_{i}(n)=0, Y_{i}(n-1)=k \mid Y_{i}(0)=i\right] \\
& =Q_{k 0} Q_{i k}^{(n-1)} \\
& =q_{k} Q_{i k}^{(n-1)}
\end{aligned}
$$

When the events $\Lambda_{i}$ and $\Gamma_{n, k}$ occur (i.e., $C$ is received over channel $i$ for the first time and correctly received over channel $k$ for its $n^{\text {th }}$ transmission), those $k-1$ packets that are received over channels $1, \cdots, k-1$ in slot $(n-1) m$ have a smaller sequence number than that associated with $C$. Since $C$ is received correctly at the end of slot $(n-1) m$, the time epoch from then until all of the above mentioned $k-1$ packets are correctly received will be the resequencing delay of $C$. If $k=1$, then there is no packet with a smaller sequence 
number than that associated with $C$ received in slot $(n-1) m$, and $C$ is not blocked by any packet in the resequencing buffer. Therefore,

$$
D_{r}=0
$$

When $k \geq 2$, we define $L$ to be the additional number of transmissions (or number of receptions including the one in slot $(n-1) m$ ) needed for all these $k-1$ packets to be received correctly. By the definition of the Markov chain $\left\{X_{M}(t): t \geq 0\right\}$, we obtain

$$
\begin{aligned}
& \mathbb{P}\left[L=l \mid \Gamma_{n, k}, \Lambda_{i}\right] \\
= & \mathbb{P}\left[X_{M}(n-1+l)=0, X_{M}(n-1+l-1) \neq 0, \cdots,\right. \\
& \left.X_{M}(n-1+1) \neq 0 \mid X_{M}(n-1)=k-1\right] \\
= & \mathbb{P}\left[X_{M}(l)=0, X_{M}(l-1) \neq 0, \cdots, X_{M}(1) \neq 0 \mid X_{M}(0)=k-1\right] \\
= & \mathbb{P}\left[X_{M}(l)=0, X_{M}(l-1) \neq 0 \mid X_{M}(0)=k-1\right] \\
= & \begin{cases}P_{k-1,0}, & \text { if } l=1, \\
\sum_{j=1}^{k-1} P_{k-1, j}^{(l-1)} P_{j 0}, & \text { if } l \geq 2 .\end{cases}
\end{aligned}
$$

When the events $\Lambda_{i}$ and $\Gamma_{n, k}$ occur with $k \geq 2$, we have

$$
D_{r}=m(L-1)
$$


By conditioning on the events $\Lambda_{i}$ and $\Gamma_{n, k}$, we have

$$
\begin{aligned}
\mathbb{P}\left[D_{r}=0\right]= & \sum_{i=1}^{M} \sum_{n=1}^{\infty} \sum_{k=1}^{i} \mathbb{P}\left[D_{r}=0 \mid \Gamma_{n, k}, \Lambda_{i}\right] \mathbb{P}\left[\Gamma_{n, k} \mid \Lambda_{i}\right] \mathbb{P}\left[\Lambda_{i}\right] \\
= & \sum_{i=1}^{M} \mathbb{P}\left[\Lambda_{i}\right]\left(\sum_{n=1}^{\infty} \mathbb{P}\left[D_{r}=0 \mid \Gamma_{n, 1}, \Lambda_{i}\right] \mathbb{P}\left[\Gamma_{n, 1} \mid \Lambda_{i}\right]+\right. \\
& \left.\sum_{n=1}^{\infty} \sum_{k=2}^{i} \mathbb{P}\left[L=1 \mid \Gamma_{n, k}, \Lambda_{i}\right] \mathbb{P}\left[\Gamma_{n, k} \mid \Lambda_{i}\right]\right) \\
= & \sum_{i=1}^{M} \mathbb{P}\left[\Lambda_{i}\right] \sum_{n=1}^{\infty}\left(\mathbb{P}\left[\Gamma_{n, 1} \mid \Lambda_{i}\right]+\sum_{k=2}^{i} P_{k-1,0} \mathbb{P}\left[\Gamma_{n, k} \mid \Lambda_{i}\right]\right) \\
= & \sum_{i=1}^{M} \mathbb{P}\left[\Lambda_{i}\right] \sum_{k=1}^{i} P_{k-1,0}\left(\sum_{n=1}^{\infty} \mathbb{P}\left[\Gamma_{n, k} \mid \Lambda_{i}\right]\right),
\end{aligned}
$$

where the second, third and the last equalities are due to (4.27), (4.29) and (4.28), and $P_{00}=1$, respectively. Similarly, for $x=1,2, \cdots$, we obtain

$$
\begin{aligned}
\mathbb{P}\left[D_{r}=m x\right] & =\sum_{i=1}^{M} \sum_{n=1}^{\infty} \sum_{k=1}^{i} \mathbb{P}\left[D_{r}=m x \mid \Gamma_{n, k}, \Lambda_{i}\right] \mathbb{P}\left[\Gamma_{n, k} \mid \Lambda_{i}\right] \mathbb{P}\left[\Lambda_{i}\right] \\
& =\sum_{i=2}^{M} \sum_{k=2}^{i} \sum_{n=1}^{\infty} \mathbb{P}\left[L=x+1 \mid \Gamma_{n, k}, \Lambda_{i}\right] \mathbb{P}\left[\Gamma_{n, k} \mid \Lambda_{i}\right] \mathbb{P}\left[\Lambda_{i}\right] \\
& =\sum_{i=2}^{M} \mathbb{P}\left[\Lambda_{i}\right] \sum_{k=2}^{i}\left(\sum_{j=1}^{k-1} P_{k-1, j}^{(x)} P_{j 0}\right)\left(\sum_{n=1}^{\infty} \mathbb{P}\left[E_{n, k} \mid \Lambda_{i}\right]\right) .
\end{aligned}
$$

For $i, k \geq 1$, by (4.25) and (4.26), we have

$$
\begin{aligned}
\sum_{n=1}^{\infty} \mathbb{P}\left[\Gamma_{n, k} \mid \Lambda_{i}\right] & =q_{k}\left(\sum_{n=1}^{\infty} Q_{i k}^{(n)}+\mathbb{I}_{\{k=i\}}\right) \\
& =q_{k}\left(\sum_{n=1}^{\infty} \mathbf{T}^{(n)}+\mathbf{I}\right)_{i k} \\
& =q_{k}\left(\sum_{n=0}^{\infty} \mathbf{T}^{n}\right)_{i k} \\
& =q_{k} S_{i k},
\end{aligned}
$$

from which we obtain (4.24) using (4.30) and (4.31). Theorem 4.3 is proved. 


\subsection{Analysis for Markov Channels}

In this section, we analyze the distribution function of the resequencing buffer occupancy and the mean resequencing delay for Markov channels. That is, the packet error property of channel $i$, for $i=1, \cdots, M$, is characterized by the error process $\left\{E_{i}(k): k \geq 0\right\}$, which is a two-state discrete-time Markov chain. $k$ represents the very beginning of slot $k m$. The $M$ error processes are mutually independent and have the same state space and transition matrix given by (2.1) and (2.2), respectively. We assume that, before the transmission of a block of $M$ packets, which occurs during $k^{\text {th }}$ step (or in slot $k m$ ), the transmitter knows the realizations (or observed values) of the random variables $E_{i}(k)$, for $i=1, \cdots, M$.

The dynamic scheduling works as follows. The transmitter counts the number, referred to as $L$, of channels whose states at step $t+1$ are in "GOOD". If $L$ is either zero or $M$ (i.e., all channels are in a same state), each packet of the $M$ packets for transmitting at step $t+1$ is randomly (i.e., with probability $1 / M$ ) assigned to a channel; otherwise (i.e., $L$ is between 0 and $M$ ), the transmitter assigns each of the packets with sequence numbers being the first $L$ smallest in the block of $M$ packets, to a channel, whose state is in "GOOD", and assigns the rest $(M-L)$ packets to the channels with states being in “ $B A D ”$.

We let $C_{o}$ be the packet that has the smallest sequence number in the block of $M$ packets to be received at the end of the slot of observing the resequencing buffer, and then let $B_{r}$ and $A_{n}$ have the same definitions as given in Section 4.1.

If $A_{0}$ is true, the resequencing buffer is empty at the observation instant. When $A_{n}$ is true for $n \geq 1$, we denote by $n m$ the slot at the end of which $C_{o}$ is received for 
$(n+1)^{s t}$ time, and by $B_{r, n}$ the resequencing buffer occupancy. For each $t=0, \cdots, n$, we define $X(t)$ as the number of packets, in the block of $M$ packets received at the end of slot $t m$, which have a sequence number smaller than that associated with $C_{o}$, and $Y(t)$ as the number of channels whose states are in " $B A D$ " at step $t$. Clearly, $X(t)$ is a $\{0,1, \cdots, M-1\}$-valued random variable and $Y(t)$ is a $\{0,1, \cdots, M\}$-valued random variable. Furthermore, the process $\{(X(t), Y(t)): t=0,1, \cdots n\}$ is a Markov chain with the state space

$$
\Theta=\{0,1, \cdots, \mathbf{M}-\mathbf{1}\}
$$

where

$$
\mathbf{i}=\{(i, 0),(i, 1), \cdots,(i, M)\}, \quad i=0,1, \cdots, M-1
$$

is a vector. In the following, we use $\left(\begin{array}{l}x \\ y\end{array}\right)$ to represent the binomial coefficient of $x$ choosing $y$, and define

$$
\begin{aligned}
\bar{h} & =M-h ; & \\
\bar{e}_{G} & =1-e_{G}, & \bar{e}_{B}=1-e_{B} \\
\bar{\alpha} & =1-\alpha ; & \bar{\beta}=1-\beta .
\end{aligned}
$$

The transition probability matrix $\mathbf{P}$ of the Markov chain $\{(X(t), Y(t)): t=0,1, \cdots n\}$ is given by

$$
\mathbf{P}=\left[\begin{array}{lllll}
\mathbf{P}_{0,0} & \mathbf{O} & \mathrm{O} & \cdots & \mathbf{O} \\
\mathbf{P}_{1,0} & \mathbf{P}_{1,1} & \mathrm{O} & \cdots & \mathbf{O} \\
\mathbf{P}_{2,0} & \mathbf{P}_{2,1} & \mathbf{P}_{2,2} & \cdots & \mathbf{O} \\
\vdots & \vdots & \vdots & \ddots & \mathbf{O} \\
\mathbf{P}_{M-1,0} & \mathbf{P}_{M-1,1} & \mathbf{P}_{M-1,2} & \cdots & \mathbf{P}_{M-1, M-1}
\end{array}\right]
$$


where, $\mathrm{O}$ represents the zero matrix,

$$
P_{(0, h),(0, k)}=\sum_{l=0}^{h}\left(\left(\begin{array}{l}
h \\
l
\end{array}\right) \bar{\beta}^{l} \beta^{h-l}\left(\begin{array}{c}
\bar{h} \\
k-l
\end{array}\right) \alpha^{k-l} \bar{\alpha}^{\bar{h}+l-k}\right)
$$

and,

$$
P_{(i, h),(j, k)}=f(i, j, h) P_{(0, h),(0, k)}
$$

for which

$$
f(i, j, h)= \begin{cases}\left(\begin{array}{c}
i \\
j
\end{array}\right) e_{G}^{j} \bar{e}_{G}^{i-j}, & \text { if } i \leq \bar{h}, \\
\sum_{l=0}^{j}\left(\begin{array}{c}
\bar{h} \\
l
\end{array}\right) e_{G}^{l} \bar{e}_{G}^{\bar{h}-l}\left(\begin{array}{c}
i-\bar{h} \\
j-l
\end{array}\right) e_{B}^{j-l} \bar{e}_{B}^{i-\bar{h}+l-j}, & \text { else, }\end{cases}
$$

for $1 \leq i \leq M-1,0 \leq j \leq i$ and $0 \leq h, k \leq M$. The derivation of the transition matrix $\mathbf{P}$ is given as follows.

It is clear that the process $\{Y(t): t=0,1, \cdots n\}$ is a recurrent Markov chain. Since the state space of the Markov chain is finite, it is ergodic [19]. The Markov chain $\{Y(t): t=0,1, \cdots n\}$ is in steady state when the system is steady. For $h, k=0, \cdots, M$ and $h \leq k$, we compute the transition probability $\mathbb{P}[Y(1)=k \mid Y(0)=h]$ as follows. There could be $l(=0, \cdots, h)$ channels whose states stay in " $B A D$ " from step 0 to step 1 and there are $\left(\begin{array}{l}h \\ l\end{array}\right)$ choices. Meanwhile, we need $k-l$ channels that transit from "GOOD" to " $B A D$ " in order to have $k$ channels in " $B A D$ " at step 1 , and there are $\left(\begin{array}{c}M-h \\ k-l\end{array}\right)$ choices. Therefore,

$$
\begin{aligned}
& \mathbb{P}[Y(1)=k \mid Y(0)=h]= \\
& \sum_{l=0}^{h}\left(\begin{array}{l}
h \\
l
\end{array}\right) \beta^{l}(1-\beta)^{h-l}\left(\begin{array}{c}
M-h \\
k-l
\end{array}\right)(1-\alpha)^{k-l} \alpha^{(M-h)-(k-l)}
\end{aligned}
$$

with the binomial coefficient rule

$$
\left(\begin{array}{l}
x \\
y
\end{array}\right)=0, \text { if } y<0 \text { or } x<y
$$


being applied, and this transition probability is true for any $h, k=0, \cdots, M$. By the definition of $X(t)$, it will never be different from state 0 once $X(t)$ enters the state. Then the transition probability $P_{(0, h),(0, k)}$ is simply equal to the transition probability $\mathbb{P}[Y(1)=$ $k \mid Y(0)=h]$ of the Markov chain $\{Y(t): t=0,1, \cdots n\}$. That is, the matrix block $\mathbf{P}_{\mathbf{0 0}}$ in (4.37) is the transition matrix of the Markov chain $\{Y(t): t=0,1, \cdots n\}$. For example, the matrix block $\mathbf{P}_{00}$ for $M=2$ is

$$
\mathbf{P}_{\mathbf{0 0}}=\left[\begin{array}{ccc}
\alpha^{2} & 2 \alpha \bar{\alpha} & \bar{\alpha}^{2} \\
\alpha \bar{\beta} & \alpha \beta+\bar{\alpha} \bar{\beta} & \bar{\alpha} \beta \\
\bar{\beta}^{2} & 2 \beta \bar{\beta} & \beta^{2}
\end{array}\right]
$$

or for $M=4$ is

$$
\mathbf{P}_{\mathbf{0 0}}=\left[\begin{array}{lllll}
\alpha^{4} & 4 \alpha^{3} \bar{\alpha} & 6 \alpha^{2} \bar{\alpha}^{2} & 4 \alpha \bar{\alpha}^{3} & \bar{\alpha}^{4} \\
\alpha^{3} \bar{\beta} & 3 \alpha^{2} \bar{\alpha} \bar{\beta}+\alpha^{3} \beta & 3 \alpha \bar{\alpha}^{2} \bar{\beta}+3 \alpha^{2} \bar{\alpha} \beta & 3 \alpha \bar{\alpha}^{2} \beta+\bar{\alpha}^{3} \bar{\beta} & \bar{\alpha}^{3} \beta \\
\alpha^{2} \bar{\beta}^{2} & 2 \alpha \bar{\alpha} \bar{\beta}^{2}+2 \alpha^{2} \beta \bar{\beta} & \alpha^{2} \beta^{2}+\bar{\alpha}^{2} \bar{\beta}^{2}+ & 2 \bar{\alpha}^{2} \beta \bar{\beta}+2 \alpha \bar{\alpha} \beta^{2} & \bar{\alpha}^{2} \beta^{2} \\
& & 4 \alpha \bar{\alpha} \beta \bar{\beta} & \\
\alpha \bar{\beta}^{3} & 3 \alpha \beta \bar{\beta}^{2}+\bar{\alpha} \bar{\beta}^{3} & 3 \bar{\alpha} \beta \bar{\beta}^{2}+3 \alpha \beta^{2} \bar{\beta} & 3 \bar{\alpha} \beta^{2} \bar{\beta}+\alpha \beta^{3} & \bar{\alpha} \beta^{3} \\
\bar{\beta}^{4} & 4 \beta \bar{\beta}^{3} & 6 \beta^{2} \bar{\beta}^{2} & 4 \beta^{3} \bar{\beta} & \beta^{4}
\end{array}\right] .
$$

For each $i=1, \cdots, M-1, j=0, \cdots, i$, and $0 \leq h, k \leq M$, we use $P_{(i, h),(j, k)}$ to represent the transition probability $\mathbb{P}[(X(1), Y(1))=(j, k) \mid(X(0), Y(0))=(i, h)]$. By the definition of $X(t)$ and $Y(t), X(1)$ is independent of $Y(1)$ given $X(0)=i$ and 
$Y(0)=h$, and $Y(1)$ is independent of $X(0)$ given $Y(0)=h$. Therefore,

$$
\begin{aligned}
& \mathbb{P}[(X(1), Y(1))=(j, k) \mid(X(0), Y(0))=(i, h)] \\
= & \mathbb{P}[X(1)=j \mid Y(1)=k,(X(0), Y(0))=(i, h)] \mathbb{P}[Y(1)=k \mid(X(0), Y(0))=(i, h)] \\
= & \mathbb{P}[X(1)=j \mid(X(0), Y(0))=(i, h)] P_{(0, h),(0, k)} .
\end{aligned}
$$

The event $\{(X(0), Y(0))=(i, h)\}$ is equivalent to the fact that, $i$ packets among the $M$ received in slot 0 have a sequence number smaller than that associated with $C_{o}$, and when these $i$ packets are received, there are $h$ channels whose states are in "BAD". If $i \leq$ $\bar{h}=M-h$, these $i$ packets are all received over channels whose states are in "GOOD" due to the dynamic scheduling. By the assumption of mutual independence between different channels, the conditional probability $\mathbb{P}[X(1)=j \mid(X(0), Y(0))=(i, h)]$ is of a binomial distribution with parameter $e_{G}$ and $i$, i.e.,

$$
\mathbb{P}[X(1)=j \mid(X(0), Y(0))=(i, h)]=\left(\begin{array}{l}
i \\
j
\end{array}\right) e_{G}^{j}\left(1-e_{G}\right)^{i-j}
$$

On the other hand, if $i>\bar{h}$, then $\bar{h}$ packets of sequence numbers smaller than that associated with $C_{o}$ are received over channels whose states are in "GOOD", and the rest $i-\bar{h}$ packets are over channels in " $B A D$ ". The conditional probability $\mathbb{P}[X(1)=j \mid$ $(X(0), Y(0))=(i, h)]$ is,

$$
\begin{aligned}
& \mathbb{P}[X(1)=j \mid(X(0), Y(0))=(i, h)] \\
& =\sum_{l=0}^{j}\left(\begin{array}{l}
\bar{h} \\
l
\end{array}\right) e_{G}^{l}\left(1-e_{G}\right)^{\bar{h}-l}\left(\begin{array}{c}
i-\bar{h} \\
j-l
\end{array}\right) e_{B}^{j-l}\left(1-e_{B}\right)^{i-\bar{h}+l-j} .
\end{aligned}
$$

The derivation of the transition matrix $\mathbf{P}$ given in (4.37) is complete.

We note that, with the same argument for the derivations of (4.46) and (4.47) above, 
(4.39) is true, and so is (4.40), for $i=M$ and $0 \leq j, h, k \leq M$. (4.39) for $i=M$ will be used for the derivation of (4.50), and (4.40) for $i=M$ will be used in (4.56).

We denote by $\pi=\left(\pi_{0}, \pi_{1}, \cdots, \pi_{M}\right)$ the stationary probability distribution of the Markov chain $\{Y(t): t \geq 0\}$. The pgf of $B_{r, n}$ is given in the following lemma.

Lemma 4.4. If $C_{o}$ has been (incorrectly) received for $n$ times until the observation instant when $n \geq 1$, the probability generating function of $B_{r, n}$ is

$$
\begin{aligned}
\mathcal{G}_{B_{r, n}}(z)= & \frac{1}{\mathbb{P}\left[A_{n}\right]} \sum_{\left(x_{0}, y_{0}\right) \in \Theta} \cdots \sum_{\left(x_{n-1}, y_{n-1}\right) \in \Theta} \sum_{y_{n}=0}^{M} \\
& \prod_{l=0}^{n-1} \mathcal{G}_{B_{r, n}^{(l)}}(z) \mathbb{P}\left[C\left(n, x_{0}, y_{0}, \cdots, x_{n-1}, y_{n-1}, 0, y_{n}\right)\right],
\end{aligned}
$$

where, for $l=0, \cdots, n-1$,

$$
\mathcal{G}_{B_{r, n}^{(l)}}(z)= \begin{cases}\left.\left(e_{B}+z \bar{e}_{B}\right)\right)^{M-1-x_{l}}, & \text { if } x_{l}+y_{l} \geq M-1 \\ \left(e_{B}+z \bar{e}_{B}\right)^{y_{l}}\left(e_{G}+z \bar{e}_{G}\right)^{M-1-x_{l}-y_{l}}, & \text { else, }\end{cases}
$$

and,

$$
\begin{gathered}
\mathbb{P}\left[C\left(n, x_{0}, y_{0}, \cdots, x_{n-1}, y_{n-1}, 0, y_{n}\right)\right]=\left(\prod_{l=0}^{n-1} \delta_{l}\right)\left(\sum_{y=0}^{M} \pi_{y} \sum_{x=0}^{x_{0}} P_{(M, y),\left(x, y_{0}\right)}\right) \times \\
P_{\left(x_{0}, y_{0}\right),\left(x_{1}, y_{1}\right)} \cdots P_{\left(x_{n-1}, y_{n-1}\right),\left(0, y_{n}\right)},
\end{gathered}
$$

in which

$$
\delta_{l}= \begin{cases}e_{B}, & \text { if } x_{l}+y_{l}>M-1 \\ e_{G}, & \text { else }\end{cases}
$$

for $l=0, \cdots, n-1$. 
Proof : By the definition of $X(t), X(n)=0$. By conditioning on a sample path of the the Markov chain $\{(X(t), Y(t)): t=0,1, \cdots n\}$, we have

$$
\begin{aligned}
& \mathcal{G}_{B_{r, n}}(z) \\
= & \sum_{\left(x_{0}, y_{0}\right) \in \Theta} \ldots \sum_{\left(x_{n-1}, y_{n-1}\right) \in \Theta} \sum_{y_{n}=0}^{M} \mathbb{E}\left[z^{B_{r}} \mid A_{n}, B\left(x_{0}, y_{0}, \cdots, x_{n-1}, y_{n-1}, 0, y_{n}\right)\right] \\
& \mathbb{P}\left[B\left(x_{0}, y_{0}, \cdots, x_{n-1}, y_{n-1}, 0, y_{n}\right) \mid A_{n}\right] \\
= & \frac{1}{\mathbb{P}\left[A_{n}\right]} \sum_{\left(x_{0}, y_{0}\right) \in \Theta} \cdots \sum_{\left(x_{n-1}, y_{n-1}\right) \in \Theta} \sum_{y_{n}=0}^{M} \mathbb{E}\left[z^{B_{r}} \mid C\left(n, x_{0}, y_{0}, \cdots, x_{n-1}, y_{n-1}, 0, y_{n}\right)\right] \\
& \mathbb{P}\left[C\left(n, x_{0}, y_{0}, \cdots, x_{n-1}, y_{n-1}, 0, y_{n}\right)\right],
\end{aligned}
$$

where the event $C\left(n, x_{0}, y_{0}, \cdots, x_{n-1}, y_{n-1}, 0, y_{n}\right)$ occurs if and only if the following three independent events occur.

$A_{n}^{(1)}$ : at most $x_{0}$ packets in the $M$ packets received in slot $-m$ (i.e., $m$ slot before slot 0 ) are packets retransmitted in slot 0 , which guarantees that $C_{o}$ is received for the first time in slot 0 with $x_{0}$ packets having a smaller sequence number;

$A_{n}^{(2)}:$ the path of the Markov chain $\{(X(t), Y(t)): t=0,1, \cdots, n\}$ from step 0 to step $n$

$$
\begin{aligned}
& \text { is }\left\{(X(0), Y(0))=\left(x_{0}, y_{0}\right), \cdots,(X(n-1), Y(n-1))=\left(x_{n-1}, y_{n-1}\right),(X(n),\right. \\
& \left.Y(n))=\left(0, y_{n}\right)\right\}
\end{aligned}
$$

$A_{n}^{(3)}: C_{o}$ is received erroneously in slots $0, m, \cdots,(n-1) m$.

By further conditioning on $\{Y(-1)=y\}$,

$$
\begin{aligned}
\mathbb{P}\left[A_{n}^{(1)}\right] & =\sum_{x=0}^{x_{0}} \mathbb{P}\left[(X(0), Y(0))=\left(x, y_{0}\right) \mid X(-1)=M\right] \\
& =\sum_{y=0}^{M} \sum_{x=0}^{x_{0}} \pi_{y} P_{(M, y),\left(x, y_{0}\right)}
\end{aligned}
$$


where $P_{(M, y),\left(x, y_{0}\right)}$ is given by (4.39). Clearly,

$$
\mathbb{P}\left[A_{n}^{(2)}\right]=P_{\left(x_{0}, y_{0}\right),\left(x_{1}, y_{1}\right)} \cdots P_{\left(x_{n-1}, y_{n-1}\right),\left(0, y_{n}\right)}
$$

For $l=0, \cdots, n-1, C_{o}$ is received at the end of slot $l m$. In slot $l m, M-1-x_{l}$ packets with a sequence number larger than that associated with $C_{o}$ are received and $y_{l}$ channels have their error processes in " $B A D$ ". If $M-1-x_{l} \geq y_{l}$, then $C_{o}$ is received over a channel having its error process in state "GOOD". Otherwise, $C_{o}$ is received over a channel of the error process in " $B A D$ ". Thus, the probability of the event $C\left(n, x_{0}, y_{0}, \cdots, x_{n-1}, y_{n-1}, 0, y_{n}\right)$ given in (4.50) follows.

When the event $C\left(n, x_{0}, y_{0}, \cdots, x_{n-1}, y_{n-1}, 0, y_{n}\right)$ occurs, the packets that were received correctly between slot 0 and slot $(n-1) m$ and had a larger sequence number than that associated with $C_{o}$ will contribute to the resequencing buffer occupancy $B_{r, n}$. For each $l=0,1, \cdots, n-1$, we denote by $B_{r, n}^{(l)}$ the number of packets that contribute to the resequencing buffer occupancy when $C_{o}$ is received for the $(l+1)^{s t}$ time. In slot $l m$, $M-1-x_{l}$ packets with a sequence number larger than that associated with $C_{o}$ are received and $y_{l}$ channels have their error processes in " $B A D$ ". There are two cases for the distribution function of $B_{r, n}^{(l)}$. If $y_{l} \geq M-1-x_{l}$, all those $M-1-x_{l}$ packets having a larger sequence number are received over channels with the instant channel error rate $e_{B}$. Otherwise (i.e., $y_{l}<M-1-x_{l}$ ), $y_{l}$ packets among these $M-1-x_{l}$ packets are received over channels with the error rate $e_{B}$ and the rest $M-1-x_{l}-y_{l}$ packets are received over channels with the error rate $e_{G}$. By the assumption of mutual independence between different channels, $B_{r, n}^{(0)}, \cdots, B_{r, n}^{(n-1)}$ are independent Binomial random variables. There- 
fore,

$$
\begin{aligned}
\mathbb{E}\left[z^{B_{r, n}} \mid C\left(n, x_{0}, y_{0}, \cdots, x_{n-1}, y_{n-1}, 0, y_{n}\right)\right] & =\mathbb{E}\left[z^{\sum_{l=0}^{n-1} B_{r, n}^{(l)}}\right] \\
& =\prod_{l=0}^{n-1} \mathcal{G}_{B_{r, n}^{(l)}}(z),
\end{aligned}
$$

where $\mathcal{G}_{B_{r, n}^{(l)}}$ is given in (4.49) by (4.17). Lemma 4.4 is proved.

Since $A_{0}$ is true if and only if, all $M$ packets received in slot $-m$ ( $m$ slots before slot $0)$ were received successfully,

$$
\begin{aligned}
\mathbb{P}\left[A_{0}\right] & =\mathbb{P}[X(0)=0 \mid X(-1)=M] \\
& =\sum_{y=0}^{M} \mathbb{P}[X(0)=0, Y(-1)=y \mid X(-1)=M] \\
& =\sum_{y=0}^{M} \mathbb{P}[X(0)=0 \mid X(-1)=M, Y(-1)=y] \pi_{y} \\
& =\sum_{y=0}^{M} f(M, 0, y) \pi_{y},
\end{aligned}
$$

where, $f(M, 0, y)$ is given by (4.40).

By conditioning on the event $A_{n}$, the pgf $\mathcal{G}_{B_{r}}$ of the resequencing buffer occupancy $B_{r}$ is given in the following theorem.

\section{Theorem 4.5.}

$$
\begin{aligned}
\mathcal{G}_{B_{R}}(z)= & \mathbb{P}\left[A_{0}\right]+\sum_{n=1}^{\infty} \sum_{\left(x_{0}, y_{0}\right) \in \Theta} \ldots \sum_{\left(x_{n-1}, y_{n-1}\right) \in \Theta} \sum_{y_{n}=0}^{M}\left(\prod_{l=0}^{n-1} \mathcal{G}_{B_{r, n}^{(l)}}(z)\right) \\
& \mathbb{P}\left[C\left(n, x_{0}, y_{0}, \cdots, x_{n-1}, y_{n-1}, 0, y_{n}\right)\right]
\end{aligned}
$$

where $\mathbb{P}\left[A_{0}\right], \mathcal{G}_{B_{r, n}^{(l)}}(z)$, and $\mathbb{P}\left[C\left(n, x_{0}, y_{0}, \cdots, x_{n-1}, y_{n-1}, 0, y_{n}\right)\right]$ are given by (4.56), (4.49), and (4.50), respectively. 
In steady state, the mean resequencing delay $D_{r}$ of an arbitrary packet can be obtained by conditioning on $\{Y(t)=y\}$, i.e.,

$$
\mathbb{E}\left[D_{r}\right]=\sum_{y=0}^{M} \mathbb{E}\left[D_{r} \mid Y(t)=y\right] \pi_{y},
$$

where $\mathbb{E}\left[D_{r} \mid Y(t)=y\right]$ can be evaluated in the same way as that for calculating the mean resequencing delay for iid channels studied in Section 4.1.2, in which $y$ channels have the error rate $e_{B}$ and $M-y$ channels have the error rate $e_{G}$, respectively.

\subsection{Numerical and Simulation Results}

In this section, numerical and simulation analysis is carried out to provide specific properties on the system performance. Results corresponding to the dynamic scheduling policy are numerically computed, while results corresponding to the static scheduling policy are obtained through simulation. At the same time, through computations of the probability mass functions of $D_{r}$ and $B_{r}$, we demonstrate that performance values in terms of the probability mass functions of $D_{r}$ and $B_{r}$ can be efficiently obtained based on the analytical results derived in Section 4.1 and Section 4.2.

\subsubsection{Distribution Functions for Time-invariant Channels}

In this section, numerical results of the probability mass functions of the resequencing buffer occupancy and the resequencing delay are computed. We denote by $p$ the average of the error rates for the $M$ channels and by $\Delta_{i}$ the ratio of $p_{i+1}$ and $p_{i}$ for $i=1, \cdots, M-1$, or

$$
p=\frac{1}{M} \sum_{i=1}^{M} p_{i},
$$


and

$$
\Delta_{i}=\frac{p_{i+1}}{p_{i}}, i=1, \cdots, M-1
$$

We assume $\Delta=\Delta_{1}=\cdots=\Delta_{M-1}$ for any fixed channel number $M$ in this section and Section 4.3.2.

\section{Distribution Function of Resequencing Buffer Occupancy}

We consider

$$
b(x)=\mathbb{P}\left[B_{r}=x\right], x=0,1, \cdots
$$

where the pgf $\mathcal{G}_{B_{r}}(z)$ of $B_{r}$ is given in (4.9). The probability that an empty resequencing buffer is observed is

$$
b(0)=\mathcal{G}_{B_{r}}(0)
$$

Using a Cauchy contour integral, the pmf $b(x)$ for a positive integer $x$ can be expressed by

$$
b(x)=\frac{1}{2 \pi \mathrm{i}} \oint_{C_{r}} \mathcal{G}_{B_{r}}(z) z^{-x-1} d z
$$

where $\mathrm{i}$ represents the basic imaginary unit $\sqrt{-1}$ and $C_{r}$ is a circular contour around the origin with radius $0<r<1$. By sampling the integrand on $2 x$ points of $C_{r}$, the integral is approximated by [4]

$$
b(x) \approx \frac{1}{2 x r^{x}}\left\{\mathcal{G}_{B_{r}}(r)+(-1)^{x} \mathcal{G}_{B_{r}}(-r)+2 \sum_{j=1}^{x-1}(-1)^{j} \operatorname{Re}\left(\mathcal{G}_{B_{r}}\left(r e^{\pi j i / x}\right)\right)\right\}
$$

where $\operatorname{Re}(z)$ represents the real part of a complex number $z$. The error for this approximation is bounded by $r^{2 x}$. Thus, acceptable accuracy can be reached by choosing a sufficiently small $r$. Using (4.64) and (4.9), we can approximate the probability mass of $B_{r}$ for any given values of $M$ and $p_{1}, \cdots, p_{M}$. 


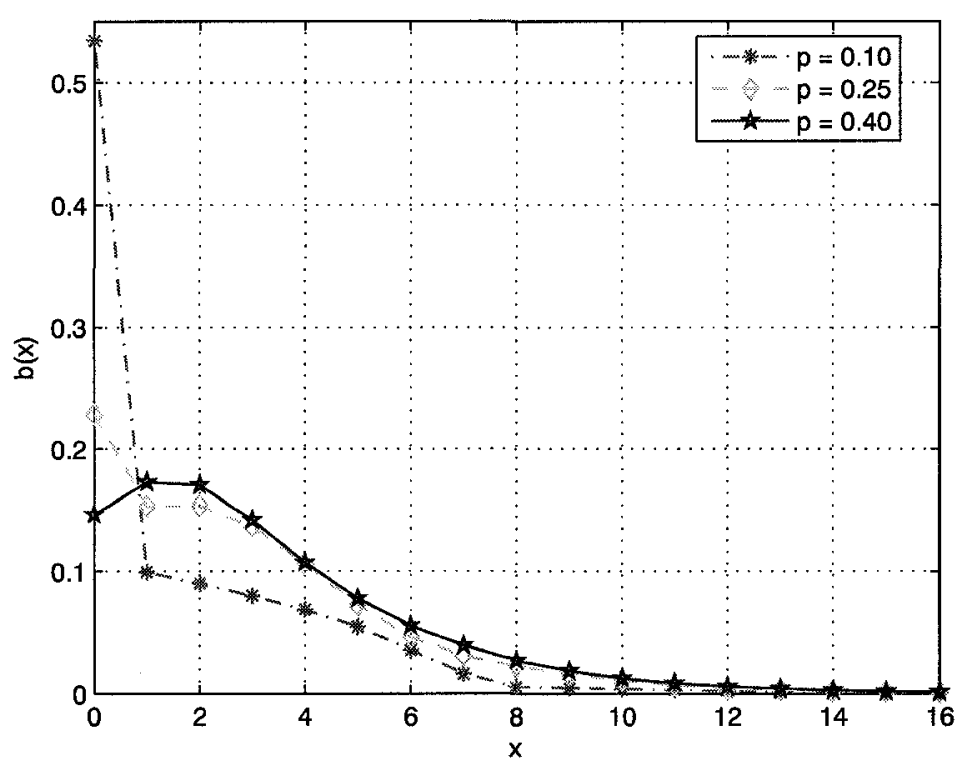

Figure 4.3: The Probability Mass Function of $B_{r}$

In Figure 4.3, we show a plot of the probability mass function of $B_{r}$ for three different average error rates. In the plot, $M=8$ and $\Delta=1.2$. As we expect, the probability that an empty resequencing buffer is observed decreases as the average error rate $p$ increases.

\section{Distribution Function of Resequencing Delay}

The distribution function of the resequencing delay for time-invariant channels is given by (4.24). In Figure 4.4, we plot the pmf of $D_{r}$ for different values of $p$. In the plot, $M=8$ and $\Delta=1.1$. As we expect, for a bigger average $p$ of the error rates, an arbitrary packet would experience a longer resequencing delay with a larger probability. In Figure 4.5 we plot the pmf of $D_{r}$ for $M=4,8$ and 16. In the plot, $p=0.25$ and $\Delta=1.1$. We observe that the steady-state probability that an arbitrary packet goes through the resequencing buffer without delay (i.e., $\mathbb{P}\left[D_{r}=0\right]$ ) decreases as $M$ increases. Intuitively, for more 


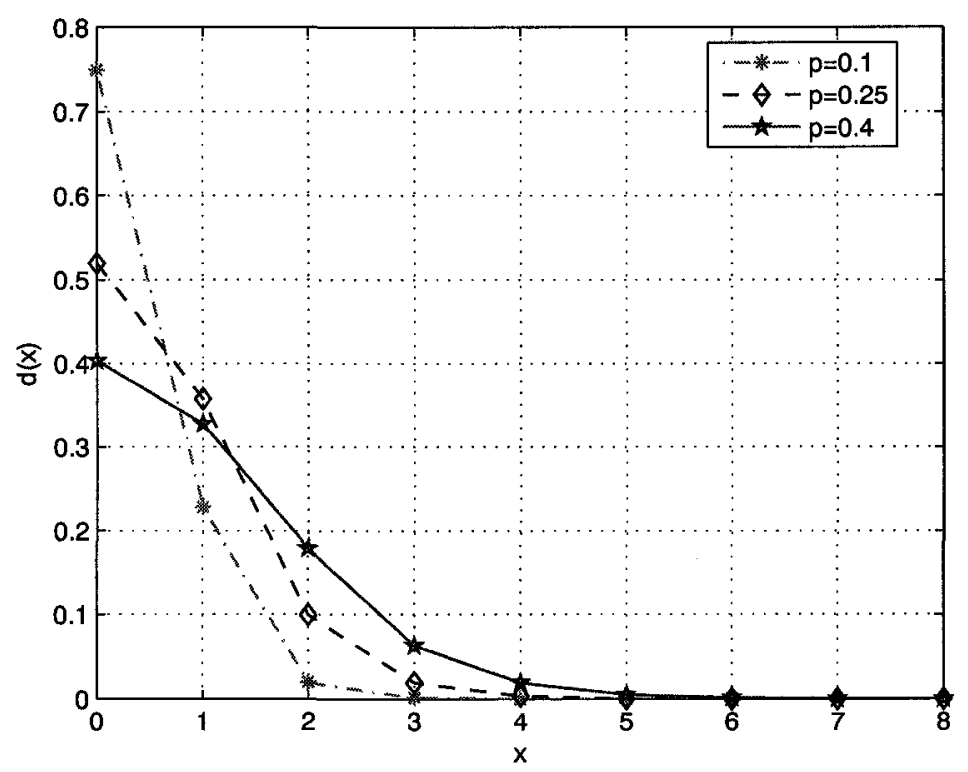

Figure 4.4: The Probability Mass Function of $D_{r}$

parallel channels, more packets are erroneously received in a slot, and an arbitrary packet will experience a longer delay in the resequencing buffer with a larger probability.

\subsubsection{Means for Time-invariant Channels}

In this section, we compute numerical values of the mean resequencing buffer occupancy $\mathbb{E}\left[B_{r}\right]$ and the mean resequencing buffer delay $\mathbb{E}\left[D_{r}\right]$ for the model with time-invariant error rates, and discuss their performance properties. 


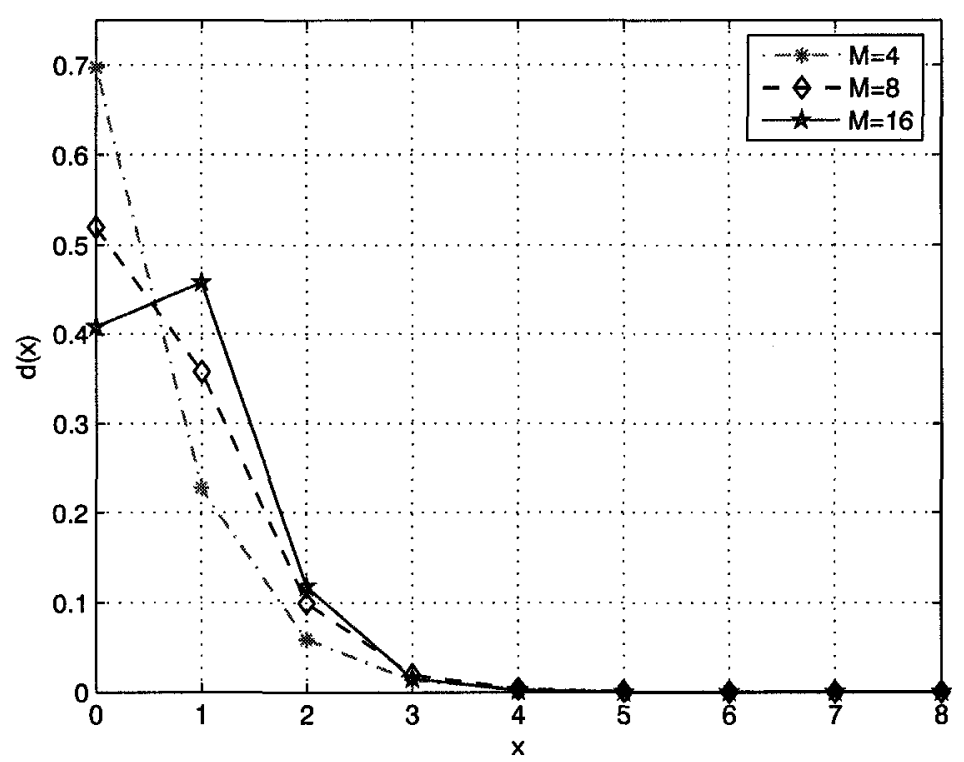

Figure 4.5: The Probability Mass Function of $D_{r}$

\section{Mean Resequencing Buffer Occupancy}

The mean resequencing buffer occupancy $\mathbb{E}\left[B_{r}\right]$ can be obtained by differentiating the pgf $\mathcal{G}_{B_{r}}(z)$ of $B_{r}$ at $z=1$, where $\mathcal{G}_{B_{r}}(z)$ is given in (4.9). Specifically,

$$
\begin{aligned}
\mathbb{E}\left[B_{r}\right] & =\left.\frac{d}{d z} \mathcal{G}_{B_{r}}(z)\right|_{z=1} \\
& =\sum_{i=1}^{M} \sum_{n=1}^{\infty} \sum_{x_{1}=0}^{i-1} \sum_{x_{2}=0}^{x_{1}} \ldots \sum_{x_{n-1}=0}^{x_{n-2}}\left(\sum_{j=1}^{M} \mu_{j} q_{j}\right) \mathbb{P}\left[C\left(n, i, x_{1}, \cdots, x_{n-1}\right)\right],
\end{aligned}
$$

where $\mu_{j}$ and $\mathbb{P}\left[C\left(n, i, x_{1}, \cdots, x_{n-1}\right)\right]$ are given in (4.11) and (4.10), respectively.

In Figure 4.6 and Figure 4.7, we plot the mean resequencing buffer occupancy $\mathbb{E}\left[B_{r}\right]$ as well as the average of the resequencing buffer occupancy for the static scheduling versus $M$ and $\Delta$, respectively. $\mathbb{E}\left[B_{r}\right]$ is numerically computed based on (4.65), while the average of the resequencing buffer occupancy for the static scheduling is obtained by 
simulation. In Figure 4.6, $\Delta=1.2, p=0.25$, and $M$ varies from 2 to 16 . As expected, the average resequencing buffer occupancy for the dynamic scheduling is smaller than that obtained from the static scheduling for each number $M$ of parallel channels, but they both increase with $M$. However, the average resequencing buffer occupancy for the static scheduling increases much faster than that for the dynamic scheduling. For instance, when $M$ is 12 , the average resequencing buffer occupancy for the static scheduling is approximately twice more than that obtained from the dynamic scheduling. In Figure 4.7, $p=0.25, M=8$, and $\Delta$ varies from 1 to 1.7 . Since $\Delta=1$ means that all channels have the same error rate $p$, the model functions without any difference between the two packet scheduling policies. Consequently, the numerical value of the mean resequencing buffer occupancy $\mathbb{E}\left[B_{r}\right]$ equals the simulation result of the average resequencing buffer occupancy for the static scheduling when $\Delta=1 . \mathbb{E}\left[B_{r}\right]$ decreases with the increase of $\Delta$, but the average resequencing buffer occupancy for the static scheduling increases with $\Delta$. Intuitively, when each channel has a larger variance in its error states, the packets transmitted over the first few channels have a larger probability to be received correctly. When the dynamic scheduling is applied, these packets have smaller sequence numbers, which results in a smaller possibility for other packets to be blocked in the resequencing buffer. In summary, the resequencing buffer occupancy benefits from the presence of the variance in the states of error rates when the dynamic scheduling is applied.

\section{Mean Resequencing Delay}

In Figure 4.8, we plot the mean resequencing delay $\mathbb{E}\left[D_{r}\right]$ for different values of $p$ and $M$. In the plot, $\Delta=1.05$. For a fixed $M$, the mean resequencing delay increases as $p$ does. Likewise, for a fixed $p$, the mean resequencing delay increases with $M$. This confirms the 


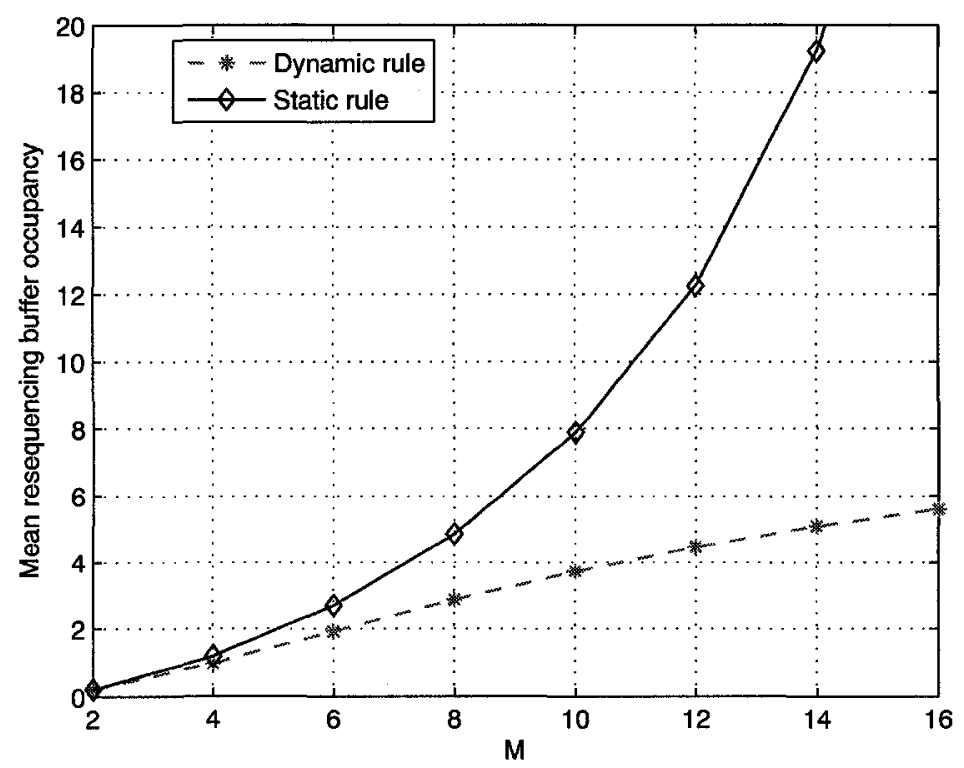

Figure 4.6: Average Resequencing Buffer Occupancy vs. $M$

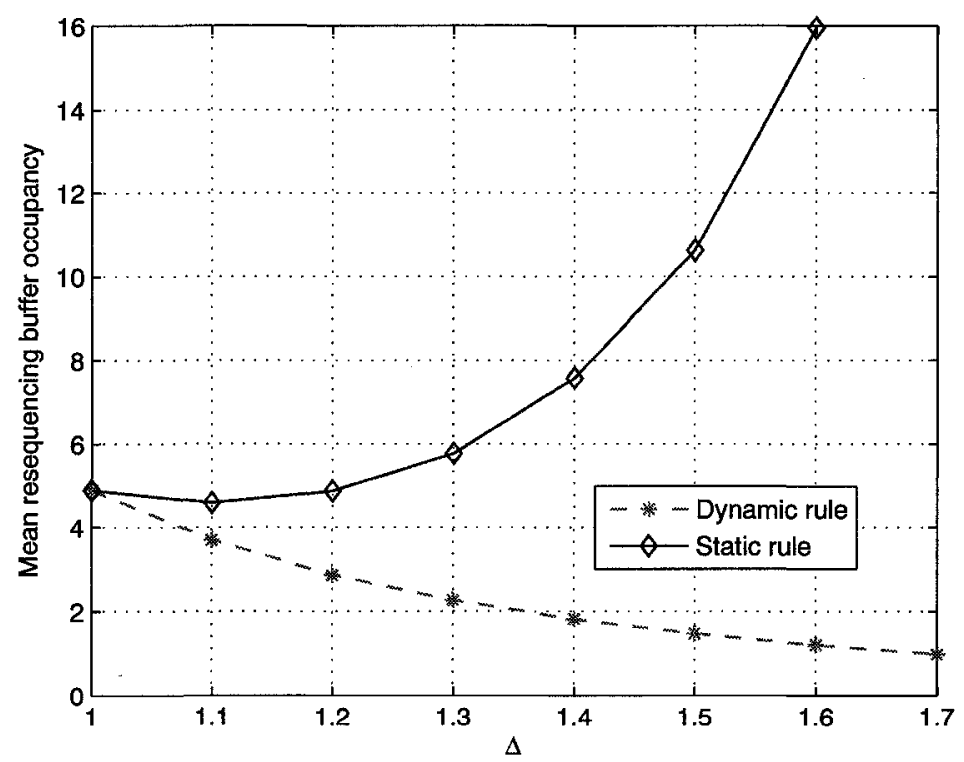

Figure 4.7: Average Resequencing Buffer Occupancy vs. $\Delta$ 


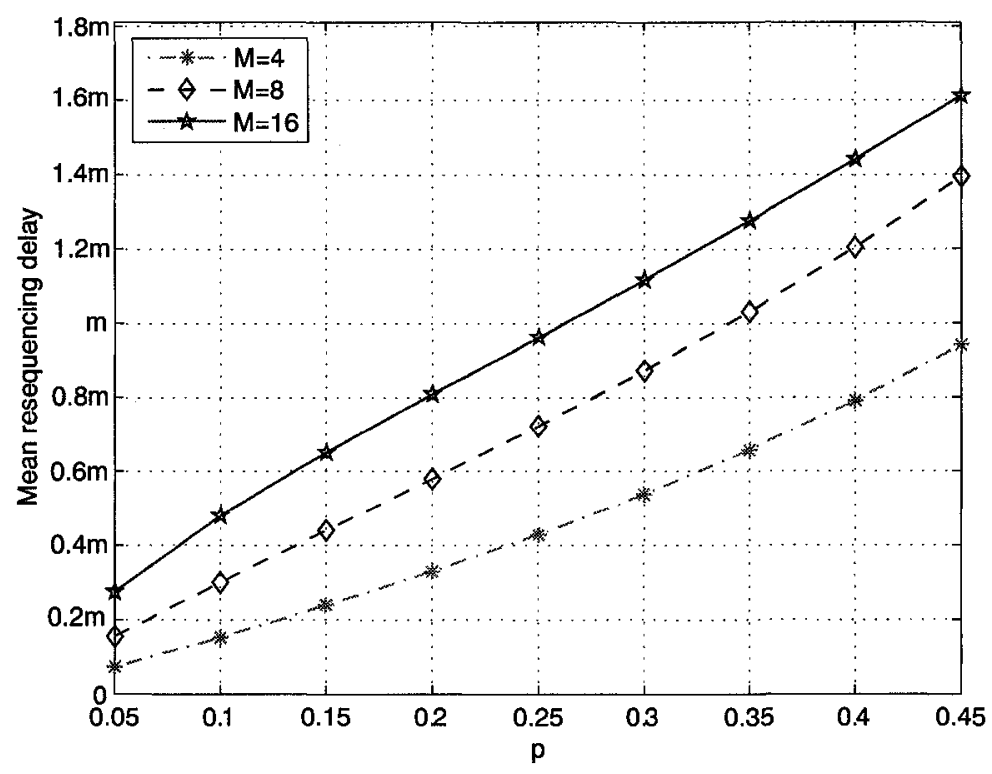

Figure 4.8: Mean Resequencing Delay

fact observed for the mean resequencing buffer occupancy, i.e., the mean resequencing buffer occupancy increases with $p$ and $M$.

\subsubsection{Means for Markov Channels}

For numerical and simulation analysis of the average resequencing buffer occupancy for Markov channels, we set

$$
K=\frac{e_{B}}{e_{G}},
$$

for a positive integer $K$ and the transition matrix given by (2.2) to be fixed as

$$
\left[\begin{array}{cc}
\alpha & 1-\alpha \\
1-\beta & \beta
\end{array}\right]=\left[\begin{array}{ll}
0.8 & 0.2 \\
0.3 & 0.7
\end{array}\right] .
$$


So the stationary distribution of the error process for each channel is given by

$$
(1-\sigma, \sigma)=(0.6,0.4)
$$

Therefore, the average packet error rate for one channel is

$$
\bar{p}=0.6 e_{G}+0.4 e_{B}=(0.6+0.4 K) e_{G} .
$$

\section{Mean Resequencing Buffer Occupancy}

In this section we denote by $\mathbb{E}\left[B_{r}\right]$ the mean resequencing buffer occupancy for Markov channels. By differentiating $\mathcal{G}_{B_{r}}(z)$ given in (4.57) at $z=1, \mathbb{E}\left[B_{r}\right]$ is

$$
\begin{gathered}
\mathbb{E}\left[B_{r}\right]=\sum_{n=1}^{\infty} \sum_{\left(x_{0}, y_{0}\right) \in \Theta} \cdots \sum_{\left(x_{n-1}, y_{n-1}\right) \in \Theta} \sum_{y_{n}=0}^{M}\left(\sum_{l=0}^{n-1} \mathbb{E}\left[B_{r, n}^{(l)}\right]\right) \\
\mathbb{P}\left[C\left(n, x_{0}, y_{0}, \cdots, x_{n-1}, y_{n-1}, 0, y_{n}\right)\right],
\end{gathered}
$$

where,

$$
\mathbb{E}\left[B_{r, n}^{(l)}\right]= \begin{cases}\left(M-1-x_{l}\right) \bar{e}_{B}, & \text { if } x_{l}+y_{l} \geq M-1, \\ y_{l} \bar{e}_{B}+\left(M-1-x_{l}-y_{l}\right) \bar{e}_{G}, & \text { else }\end{cases}
$$

for $l=0,1, \cdots, n-1$, and $\mathbb{P}\left[C\left(n, x_{0}, y_{0}, \cdots, x_{n-1}, y_{n-1}, 0, y_{n}\right)\right]$ is given in (4.50).

In Figure 4.9, Figure 4.10, and Figure 4.11, we plot numerical values of $\mathbb{E}\left[B_{r}\right]$ together with the average of the resequencing buffer occupancy for the static scheduling and for the iid channels. $\mathbb{E}\left[B_{r}\right]$ is computed based on (4.70), while the averages are obtained from simulation, and an iid channel has the error rate $\bar{p}$.

In Figure 4.9, we plot numerical values of $\mathbb{E}\left[B_{r}\right]$ for $\bar{p}=0.35, M=8$, and $K$ varying from 1 to 10 . Since $K=1$ means that the two error states of a Markov channel are equal, there is no difference between the two packet scheduling policies, which results in 
a system behavior equivalent to $M$ iid channels of the same error rate $\bar{p}=0.35$. As we expect, for $K$ larger than $1, \mathbb{E}\left[B_{r}\right]$, which is the mean resequencing buffer occupancy for Markov channels with the dynamic scheduling, is the smallest among the three curves in Figure 4.9. Moreover, as a consequence of different packet scheduling policies, the mean resequencing buffer occupancy for the dynamic scheduling decreases with $K$, while the average for the static scheduling increases with $K$. The larger the difference between the two error states (i.e., $e_{B}$ and $e_{G}$ ) is, the smaller the mean for the dynamic rule is. As shown in Figure $4.9, \mathbb{E}\left[B_{r}\right]$ will be reduced by about $75 \%$ when the ratio $e_{B} / e_{G}$ changes form 1 to 10 . In summary, with the dynamic scheduling applied, fewer packets are blocked in the resequencing buffer when the considered channel model characterizes the time-varying property in packet errors, and the mean resequencing buffer occupancy benefits from a larger variance between the two error states.

In Figure 4.10, we let $K=2, M=8$, and $\bar{p}$ vary from 0.05 to 0.45 . We observe that all three curves increase with $\bar{p}$, while the average resequencing buffer occupancy for the dynamic scheduling is less than and increases more slowly than that for the static scheduling and iid channels. In Figure 4.11, we set $K=2, \bar{p}=0.35$, and $M$ varies from 2 to 16 . As we can expect, the average resequencing buffer occupancy in each curve grows with $M$, but the average resequencing buffer occupancy for the dynamic scheduling is the smallest among the three for each value of $M$.

\section{Mean Resequencing Delay}

In Figure 4.12, we plot the mean resequencing delay for different values of $\bar{p}$ and $M$. In the plot, $K=2$. Similar to the trend observed in Section 4.3 .2 for the mean resequencing delay in the model with time-invariant error rates, $\mathbb{E}\left[D_{r}\right]$ increases as $\bar{p}$ does for a fixed 


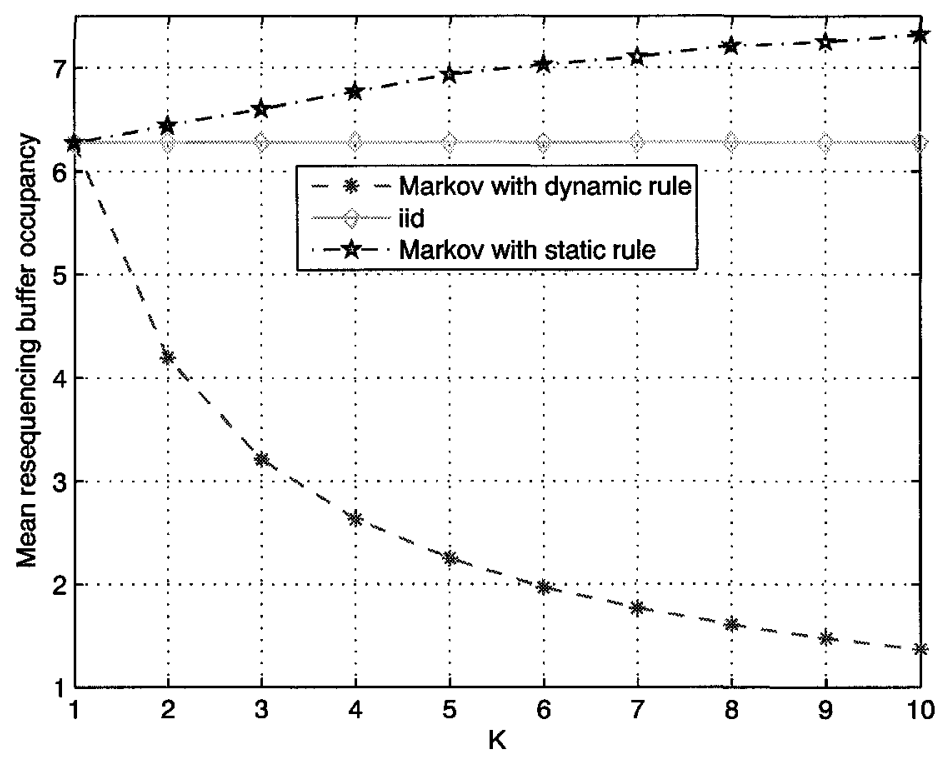

Figure 4.9: Average Resequencing Buffer Occupancy vs. $K$

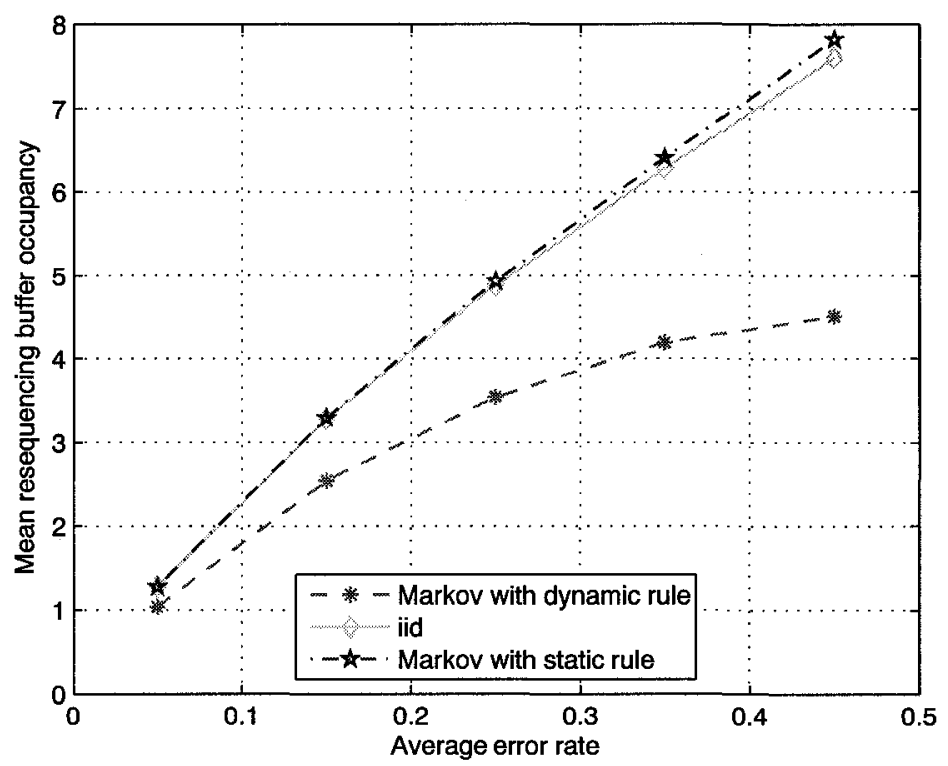

Figure 4.10: Average Resequencing Buffer Occupancy vs. $\bar{p}$ 


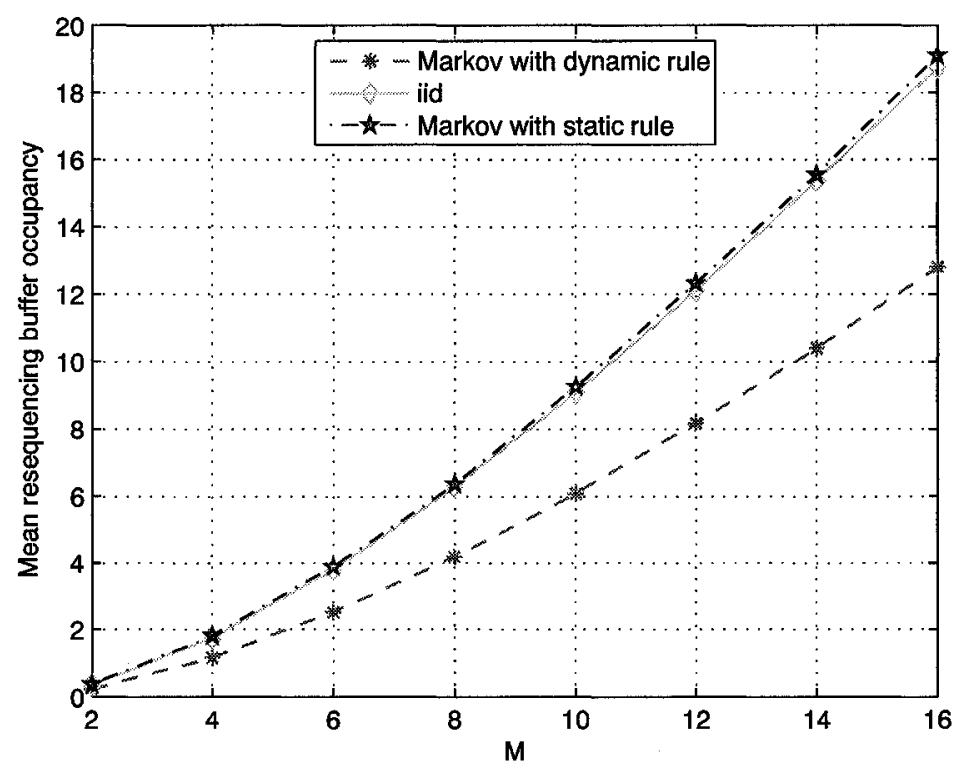

Figure 4.11: Average Resequencing Buffer Occupancy vs. $M$

number $M$, and it increases with $M$ for a fixed value of $\bar{p}$.

\subsection{Summary}

We studied the SW-ARQ-inS model for both time-invariant and time-varying channel models through analyzing the resequencing buffer occupancy and the resequencing delay, in steady state. When the channels have possibly different time-invariant error rates, we derived the pgf of the resequencing buffer occupancy and the pmf of the resequencing delay. When the Gilbert-Elliott model is assumed on each channel, we analyzed the pgf of the resequencing buffer occupancy and the mean resequencing delay. Through examples, we numerically computed the pmf of the resequencing buffer occupancy, from which we demonstrate that the pmf of the resequencing buffer occupancy can be efficiently obtained 


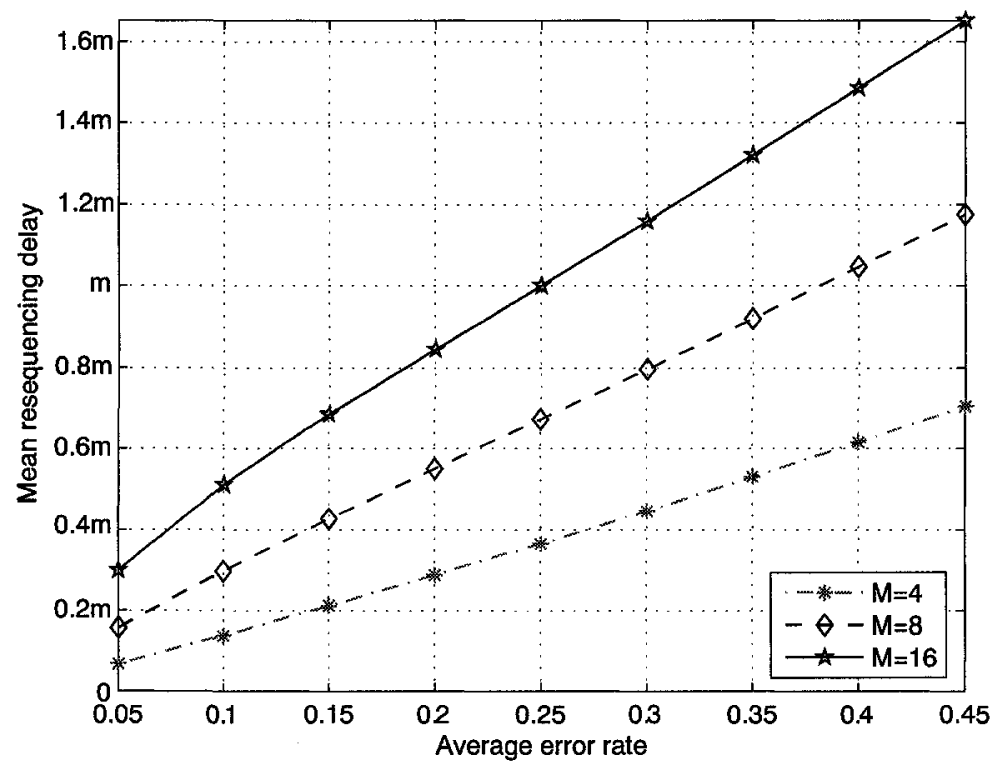

Figure 4.12: Mean Resequencing Delay

from its pgf by using the Lattice-Poisson algorithm [4], and the pmf of the resequencing delay. From numerical and simulation results, we observed that:

- the mean resequencing buffer occupancy for both time-varying and time-invariant channels benefits from the dynamic scheduling;

- the mean resequencing buffer occupancy and the mean resequencing delay for both channel models grow with the increase of either the number of channels or the average error rate of channels, but decrease with the increase of the variance in the error rates,

which means that, when the time-invariant channel error rates are assumed, the mean resequencing buffer occupancy and the mean resequencing delay decrease as the error rates of different channels become more different. Likewise, when the Gilbert-Elliott 
model is assumed, they decrease as the absolute difference between the two error states becomes larger. 


\section{Chapter 5}

\section{Packet Delay Analysis}

In this chapter, we derive the distribution function of the packet delay for MSW-ARQ and MSW-ARQ-inS in Section 5.1 and Section 5.2, respectively, when an iid model is assumed for each channel. Based on the analysis, we present numerical and simulation results in Section 5.3. Concluding remarks of this chapter are made in Section 5.4. The main results presented in this chapter have been summarized in [54] for publication.

Our analysis is under the condition that the system is in equilibrium regime. From the system settings, the condition under which the system enters equilibrium regime is trivially satisfied. Also, our steady-state analysis is based on the dynamic scheduling (simulation results for the static scheduling will be presented in Section 5.3 for performance comparisons) and the assumption that each channel assumes an iid model for packet errors. The assumption means that a time-invariant error rate $p_{i}$, which is the probability that a packet is received erroneously by the receiver or simply lost, corresponds to channel $i$, for $i=1, \cdots, M$, and $p_{i}$ might be different from $p_{j}$ for $i \neq j$.

A special case of the MSW-ARQ model for which $p_{1}=\cdots=p_{M}$ was addressed 
in [21] for the mean packet delay analysis by using a direct probabilistic argument not appropriate for a general model. Recently, the MSW-ARQ model studied in this chapter was considered and an approximate expression of the mean packet delay was obtained in [26]. However, the approximation can significantly deviate from the true result as the error rates become relatively large according to a simulation study in [26]. Compared to the throughput, which is defined as the average number of packets successfully transmitted per slot and given in [25] as

$$
\eta=\frac{\sum_{\delta=1}^{M}\left(\delta p_{\delta+1} \prod_{k=1}^{\delta} q_{k}\right)}{m}
$$

where

$$
p_{M+1}=1, \quad \text { and } \quad q_{i}=1-p_{i}, \quad i=1, \cdots, M,
$$

the packet delay is usually a more demanding performance metric.

\subsection{Packet Delay of MSW-ARQ}

In this section, steady-state analysis of the packet delay is performed for MSW-ARQ using the dynamic scheduling. Without loss of generality, the $M$ channels are numbered from channel 1 to $M$ such that $p_{1} \leq p_{2} \leq \cdots \leq p_{M}$.

In steady state, we denote by $C^{*}$ an arbitrary packet of interest and let slot 0 represent the slot at the end of which the receiver receives $C^{*}$ for the first time. We denote by $\Lambda_{i}$ the event that $C^{*}$ is received over channel $i$ for the first time, for $i=1, \cdots, M$, and $D$ the packet delay of $C^{*}$.

Provided that event $\Lambda_{i}$ occurs, we define $X_{i}(t)$ to be the number of packets, in the block of $M$ packets received at the end of slot $t m$, whose sequence numbers are not 
larger than that associated with $C^{*}$, for $t=0,1, \cdots$. An example of the stochastic process $\left\{X_{3}(t): t \geq 0\right\}$ is shown in Figure 5.1.

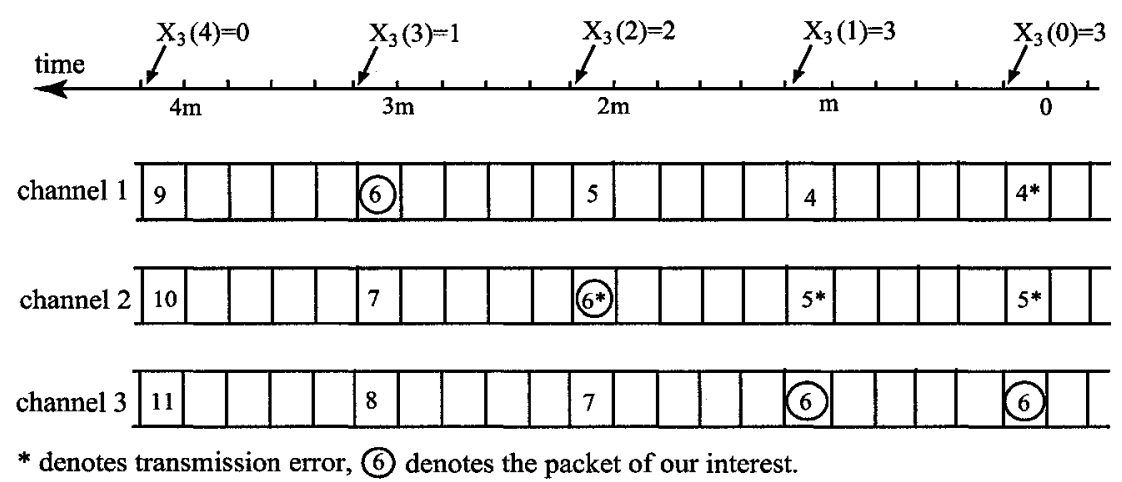

Figure 5.1: Process $\left\{X_{3}(t): t \geq 0\right\}(\mathrm{M}=3, \mathrm{~m}=5)$

The stochastic process $\left\{X_{i}(t): t=0,1, \cdots\right\}$ is a Markov chain with the state space $\{0,1, \cdots, i\}$. The initial state of the Markov chain $\left\{X_{i}(t): t=0,1, \cdots\right\}$ is $i$ with probability one, i.e.,

$$
X_{i}(0)=i, \quad i=1, \cdots, M
$$

This is because, if channel $i$ is assigned to transmit $C^{*}$, then all packets assigned to channels 1 to $i-1$ have a sequence number smaller than that associated with $C^{*}$. Also, the Markov chain $\left\{X_{i}(t): t=0,1, \cdots\right\}$ eventually enters state 0 and then stays in that state forever. Therefore, the Markov chain $\left\{X_{i}(t): t=0,1, \cdots\right\}$ is transient with absorbing state 0 and transient states $1, \cdots, i$. We define the product over an empty set $\Phi$ to be one, i.e.,

$$
\prod_{k \in \Phi} q_{k}=\prod_{k \in \Phi} p_{k}=1
$$

Since the event $\left\{X_{i}(t)=j\right\}$ is equivalent to the fact that $C^{*}$ is received in slot $t m$ over channel $j$ (i.e., packets received over channel 1 to $j$ in slot $t m$ have sequence numbers 
no larger than that associated with $C^{*}$ ), given that $C^{*}$ is received for the first time over channel $i$, we have

$$
\mathbb{P}\left[X_{i}(t+1)=0 \mid X_{i}(t)=j\right]=\prod_{k=1}^{j} q_{k}
$$

and

$$
\mathbb{P}\left[X_{i}(t+1)=l \mid X_{i}(t)=j\right]=p_{j-l+1} \prod_{k=1}^{j-l} q_{k}, j=1, \cdots, i ; l=1, \cdots, j .
$$

Given that $X_{i}(t)=j$ for $j=1, \cdots, i$, the number of packets received at the next step $(t+1)$ (or in slot $(t+1) m$ ) having a sequence number no larger than that associated with $C^{*}$ cannot be larger than $j$. Therefore, all other transition probabilities are zero. In particular, the Markov chain $\left\{X_{M}(t): t \geq 0\right\}$ has the state space $\{0,1, \cdots, M\}$ and the transition matrix

$$
\mathbf{P}=\left[\begin{array}{ccccccc}
1 & 0 & 0 & \cdots & 0 & \cdots & 0 \\
q_{1} & p_{1} & 0 & \cdots & 0 & \cdots & 0 \\
q_{1} q_{2} & p_{2} q_{1} & p_{1} & \cdots & 0 & \cdots & 0 \\
\vdots & \vdots & \vdots & \ddots & \vdots & \ddots & \vdots \\
\prod_{k=1}^{i} q_{k} & p_{i} \prod_{k=1}^{i-1} q_{k} & p_{i-1} \prod_{k=1}^{i-2} q_{k} & \cdots & p_{1} & \cdots & 0 \\
\vdots & \vdots & \vdots & \ddots & \vdots & \ddots & \vdots \\
\prod_{k=1}^{M} q_{k} & p_{M} \prod_{k=1}^{M-1} q_{k} & p_{M-1} \prod_{k=1}^{M-2} q_{k} & \cdots & p_{M-i+1} \prod_{k=1}^{M-i} q_{k} & \cdots & p_{1}
\end{array}\right]
$$

We note that the $(i+1) \times(i+1)$ upper-left submatrix of $\mathbf{P}$, which is lower triangular, is the transition matrix for the Markov chain $\left\{X_{i}(t): t \geq 0\right\}$. This implies that for $j, l$ in the state space of the Markov chain $\left\{X_{i}(t): t \geq 0\right\}$,

$$
\mathbb{P}\left[X_{i}(t+n)=l \mid X_{i}(t)=j\right]=\mathbb{P}\left[X_{M}(t+n)=l \mid X_{M}(t)=j\right]=P_{j l}^{(n)},
$$


where $P_{j l}^{(n)}$ is the $n^{\text {th }}$ step transition probability from $j$ to $l$ of the Markov chain $\left\{X_{M}(t)\right.$ : $t \geq 0\}$.

Now, we define the absorption time $\tau_{i}$ of the Markov chain $\left\{X_{i}(t): t \geq 0\right\}$, which is also the absorption time of the Markov chain $\left\{X_{M}(t): t \geq 0\right\}$ by (5.7), starting in the state $i$ at time 0 . That is,

$$
\tau_{i}=\min \left\{t: X_{M}(t)=0 \mid X_{M}(0)=i\right\}
$$

Then, we have, for $t=1$,

$$
\mathbb{P}\left[\tau_{i}=1\right]=\mathbb{P}\left[X_{M}(1)=0 \mid X_{M}(0)=i\right]=P_{i 0}
$$

and for $t \geq 2$,

$$
\begin{aligned}
\mathbb{P}\left[\tau_{i}=t\right] & =\mathbb{P}\left[X_{M}(t)=0, X_{M}(t-1) \neq 0, \cdots, X_{M}(1) \neq 0 \mid X_{M}(0)=i\right] \\
& =\sum_{j=1}^{i} \mathbb{P}\left[X_{M}(t)=0, X_{M}(t-1) \neq 0, \cdots, X_{M}(2) \neq 0 \mid X_{M}(1)=j\right] P_{i j} \\
& =\sum_{j=1}^{i} \mathbb{P}\left[X_{M}(t-1)=0, X_{M}(t-2) \neq 0, \cdots, X_{M}(1) \neq 0 \mid X_{M}(0)=j\right] P_{i j} \\
& =\sum_{j=1}^{i} \mathbb{P}\left[\tau_{j}=t-1\right] P_{i j} .
\end{aligned}
$$

Therefore, a recursive expression of the distribution function of $\tau_{i}$ is given by

$$
\mathbb{P}\left[\tau_{i}=t\right]= \begin{cases}P_{i 0}, & \text { if } t=1, \\ \sum_{k=1}^{i} \mathbb{P}\left[\tau_{k}=t-1\right] P_{i k}, & \text { if } t \geq 2 .\end{cases}
$$

Using the same argument for the derivation of (4.19), the probability that the event $\Lambda_{i}$ occurs (i.e., $C^{*}$ is received for the first time over channel $i$ ) is given by

$$
\mathbb{P}\left[\Lambda_{i}\right]=\frac{1}{1-P_{M M}} \sum_{\delta=(M+1)-i}^{M} \frac{P_{M, M-\delta}}{\delta}, i=1,2, \cdots, M,
$$


where the transition probabilities $P_{M j}$, for $j=0,1, \cdots, M$, are given in (5.6). However, $\tau_{i}$ corresponds to the total number of transmissions (equals to one plus the number of retransmissions) of $C^{*}$ before it is delivered to the upper layer given that event $\Lambda_{i}$ occurs (i.e., $C^{*}$ is received for the first time over channel $i$ ). Therefore, provided that the event $\Lambda_{i}$ occurs, the packet delay of $C^{*}$, denoted by $D_{i}$, is given by

$$
D_{i}=\left(\tau_{i}-1\right) m+1+\frac{m-1}{2}=\left(\tau_{i}-1\right) m+\frac{m+1}{2} .
$$

By conditioning on the event $\Lambda_{i}$, we have

$$
\begin{aligned}
d(x) & \equiv \mathbb{P}\left[D=\frac{m+1}{2}+m x\right] \\
& =\sum_{i=1}^{M} \mathbb{P}\left[D=\frac{m+1}{2}+m x \mid \Lambda_{i}\right] \mathbb{P}\left[\Lambda_{i}\right] \\
& =\sum_{i=1}^{M} \mathbb{P}\left[D_{i}=\frac{m+1}{2}+m x\right] \mathbb{P}\left[\Lambda_{i}\right] \\
& =\sum_{i=1}^{M} \mathbb{P}\left[\tau_{i}=x+1\right] \mathbb{P}\left[\Lambda_{i}\right], \quad x=0,1,2, \cdots
\end{aligned}
$$

After taking (5.11) and (5.12) into (5.14), the pmf of $D$ is summarized in the following theorem.

Theorem 5.1. In the multichannel system with MSW-ARQ, the steady-state probability distribution of the packet delay for an arbitrary packet is given by

$$
d(x)= \begin{cases}\frac{1}{1-P_{M M}} \sum_{i=1}^{M}\left(P_{i 0} \sum_{\delta=(M+1)-i}^{M} \frac{P_{M, M-\delta}}{\delta}\right), & \text { if } x=0, \\ \frac{1}{1-P_{M M}} \sum_{i=1}^{M}\left(\sum_{j=1}^{i} \mathbb{P}\left[\tau_{j}=x\right] P_{i j} \sum_{\delta=(M+1)-i}^{M} \frac{P_{M, M-\delta}}{\delta}\right), & \text { if } x \geq 1,\end{cases}
$$

where $P_{i j}$ and $\mathbb{P}\left[\tau_{j}=x\right]$ are given in (5.6) and (5.11), respectively. 
As is shown for deriving the pmf of the packet delay of MSW-ARQ-inS in the following section, the idea of evaluating the packet delay performance through the absorption analysis of a transient Markov chain, used for the packet delay analysis of MSW-ARQ, is a general method for delay analysis of other multichannel ARQ models.

\subsection{Packet Delay of MSW-ARQ-inS}

To derive the pmf of the packet delay of MSW-ARQ-inS, we use the same notation (e.g., packet error rates $p_{i}$ and an arbitrary packet of interest $C^{*}$ ) and definitions (e.g., the Markov chain $\left\{X_{M}(t): t=0,1, \cdots\right\}$ and its transition probability matrix $\left.\mathbf{P}\right)$ as those given in Section 4.1, but the matrix $\mathbf{P}$ is given in (4.4). We denote by $\bar{D}$ the end-to-end delay of the packet $C^{*}$, which is the amount of time between the instant at which $C^{*}$ is transmitted for the first time and the instant at which it is delivered to the upper layer. Clearly, $\bar{D}$ is the sum of the transmission time of $C^{*}$ and the resequencing time of $C^{*}$. Instead of analysis of two separate parts of the packet delay, we derive the distribution function of the packet delay for MSW-ARQ-inS through the absorption analysis method used for packet delay analysis of MSW-ARQ.

With the definition

$$
\tau_{i}=\min \left\{t: X_{M}(t)=0 \mid X_{M}(0)=i\right\}
$$

the distribution function of $\tau_{i}$ is given by

$$
\mathbb{P}\left[\tau_{i}=t\right]= \begin{cases}P_{i 0}, & \text { if } t=1 \\ \sum_{k=1}^{i} \mathbb{P}\left[\tau_{k}=t-1\right] P_{i k}, & \text { if } t \geq 2\end{cases}
$$


by using the same argument for the derivation of (5.11). In (5.17), the transition probability $P_{i k}$ is given in (4.4). Moreover, $\tau_{i}$ corresponds to the sum of the number of transmissions of $C^{*}$ and the number of cycles (one cycle consisting of $m$ slots) for which $C^{*}$ is queued in the resequencing buffer, provided that the event $\Lambda_{i}$ occurs. Therefore, $\bar{D}_{i}=\left(\tau_{i}-1\right) m+(m+1) / 2$ is the time interval between the time epoch at which $C^{*}$ is transmitted over channel $i$ for the first time and the epoch at which it is delivered from the resequencing buffer to the upper layer. That is, $\bar{D}_{i}$ is the packet delay of $C^{*}$ given that $C^{*}$ is transmitted over channel $i$ for the first time. Consequently, the steady-state distribution function $\bar{d}(x) \equiv \mathbb{P}\left[\bar{D}=\frac{m+1}{2}+m x\right]$ of the packet delay of an arbitrary packet for MSW-ARQ-inS is given by

$$
\bar{d}(x)= \begin{cases}\frac{1}{1-P_{M M}} \sum_{i=1}^{M}\left(P_{i 0} \sum_{\delta=(M+1)-i}^{M} \frac{P_{M, M-\delta}}{\delta}\right), & \text { if } x=0, \\ \frac{1}{1-P_{M M}} \sum_{i=1}^{M}\left(\sum_{j=1}^{i} \mathbb{P}\left[\tau_{j}=x\right] P_{i j} \sum_{\delta=(M+1)-i}^{M} \frac{P_{M, M-\delta}}{\delta}\right), & \text { if } x \geq 1,\end{cases}
$$

where $P_{i j}$ and $\mathbb{P}\left[\tau_{j}=x\right]$ are given in (4.4) and (5.17), respectively.

\subsection{Numerical and Simulation Results}

In this section, we compute numerically the probability mass function of the packet delay for MSW-ARQ and MSW-ARQ-inS based on the pmf derived in Section 5.1 and Section 5.2, respectively, with selected values of $M$ and $p_{i}$ 's. Then, we compare the mean packet delay performance of MSW-ARQ and MSW-ARQ-inS, and investigate the impact of packet transmission scheduling rules and system parameters on the model performance. 


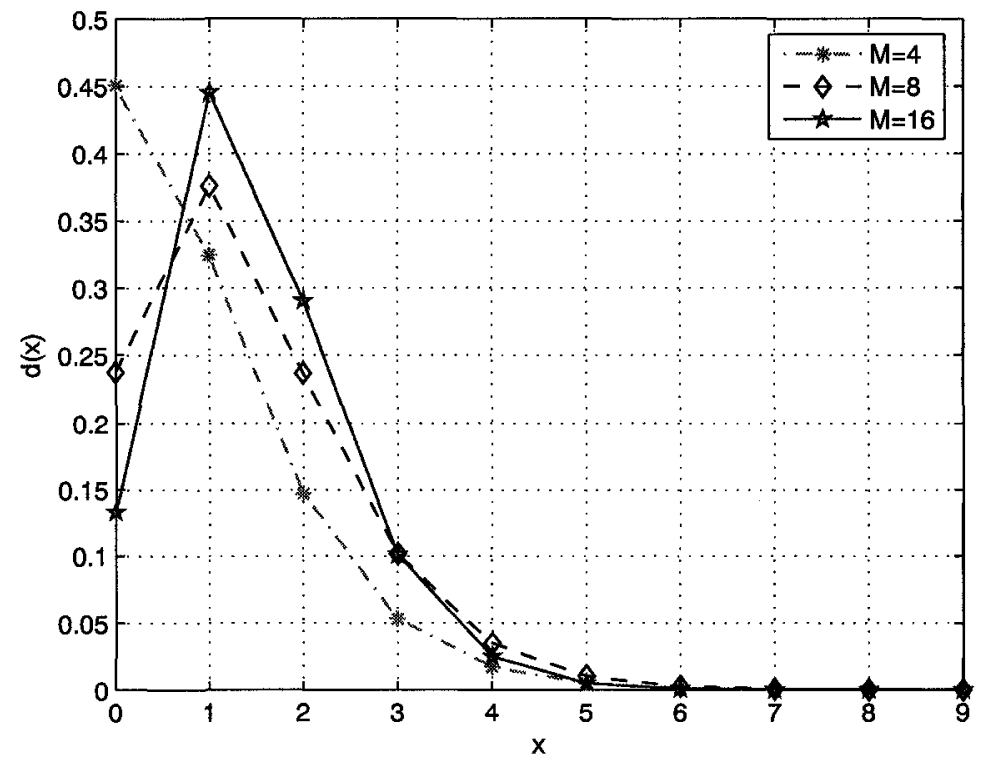

Figure 5.2: The pmf of $D$ vs. $M(\Delta=1.2, p=0.25)$

We use the same definitions of $p$ and $\Delta_{i}$ as given in Section 4.3.1. That is,

$$
p=\frac{1}{M} \sum_{i=1}^{M} p_{i},
$$

and

$$
\Delta_{i}=\frac{p_{i+1}}{p_{i}}, \quad i=1, \cdots, M-1 .
$$

We also assume $\Delta=\Delta_{1}=\cdots=\Delta_{M-1}$ in this section.

\subsubsection{Distribution Function of Packet Delay}

In Figure 5.2, Figure 5.3 and Figure 5.4, we plot the pmf of $D$ for different sets of selected values of $M, p$ and $\Delta$. We observe that the probability that a packet is delivered without retransmission (i.e., $d(0)$ ) decreases with the increase of either $M, p$ or $\Delta$. 


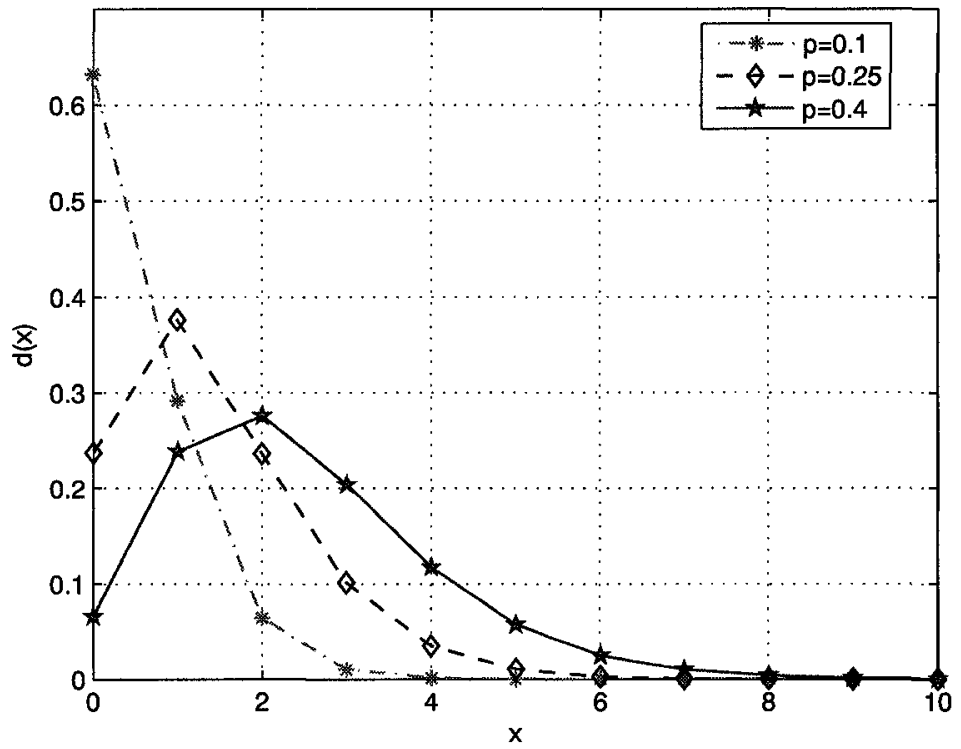

Figure 5.3: The pmf of $D$ vs. $p(M=8, \Delta=1.2)$

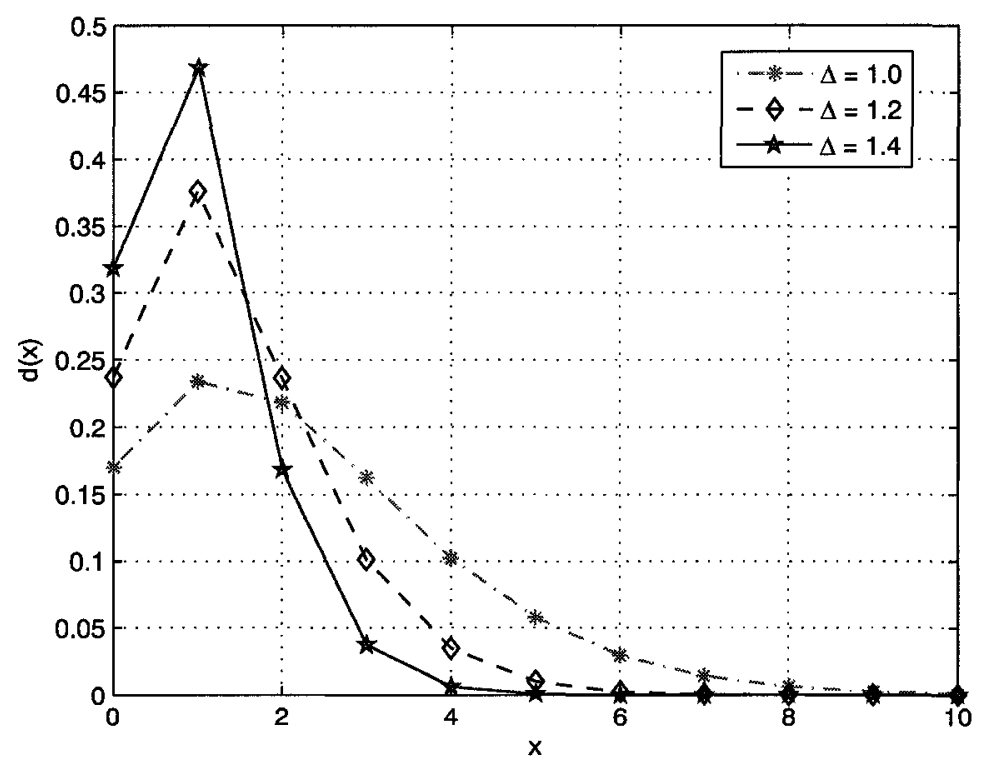

Figure 5.4: The pmf of $D$ vs. $\Delta(M=8, p=0.25)$ 


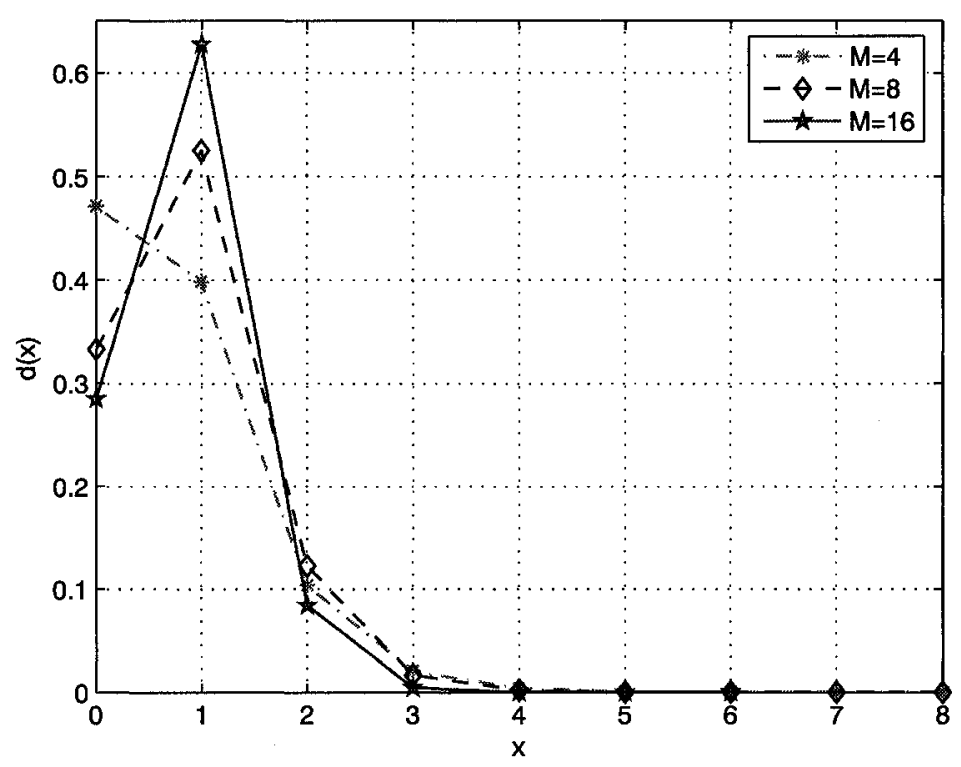

Figure 5.5: The pmf of $\bar{D}$ vs. $M(\Delta=1.2, p=0.25)$

The pmf of $\bar{D}$ is plotted in Figure 5.5, Figure 5.6 and Figure 5.7 for different sets of values of the parameters, respectively. Similar to the observation for the pmf of $D$, the probability $\bar{d}(0)$ decreases as either one of the three parameters increases.

\subsubsection{Performance Comparison of MSW-ARQ and MSW-ARQ-inS}

In Figure 5.8, Figure 5.9 and Figure 5.10, we plot numerical values of the expectations $\mathbb{E}[D]$ and $\mathbb{E}[\bar{D}]$ together with the averages of the packet delay with the static scheduling. $\mathbb{E}[D]$ and $\mathbb{E}[\bar{D}]$ are numerically computed using the corresponding probability mass function obtained in Section 5.1 and in Section 5.2, respectively. The average packet delay for MSW-ARQ and MSW-ARQ-inS with the static scheduling is obtained from simulation. In the following figures, $m$ is set to be 5 . 


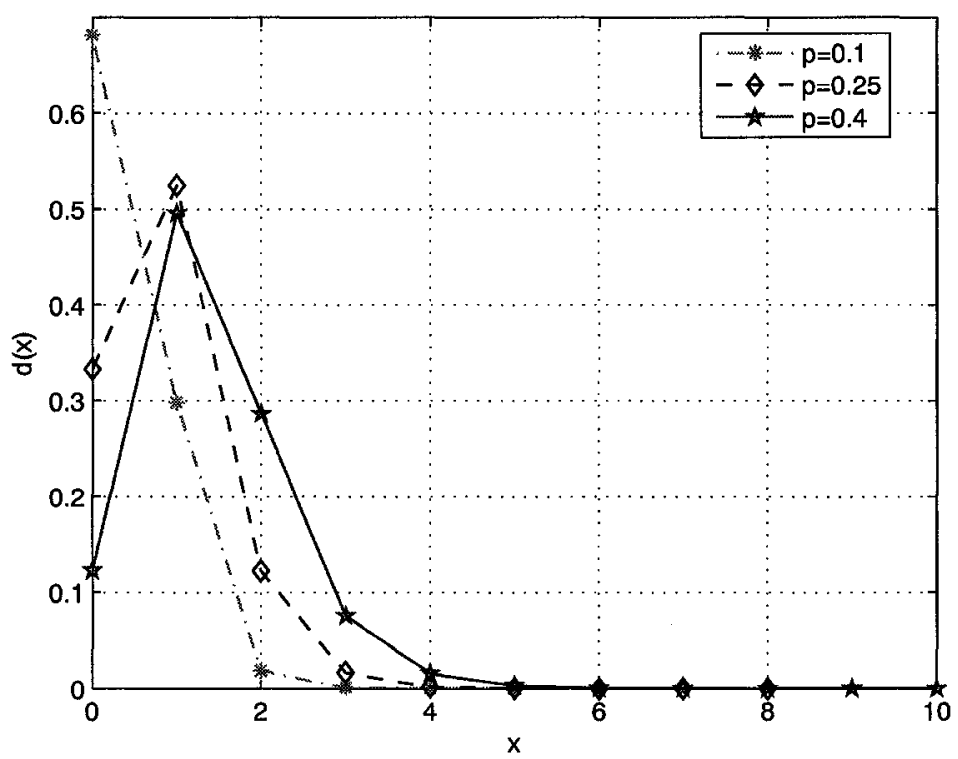

Figure 5.6: The pmf of $\bar{D}$ vs. $p(M=8, \Delta=1.2)$

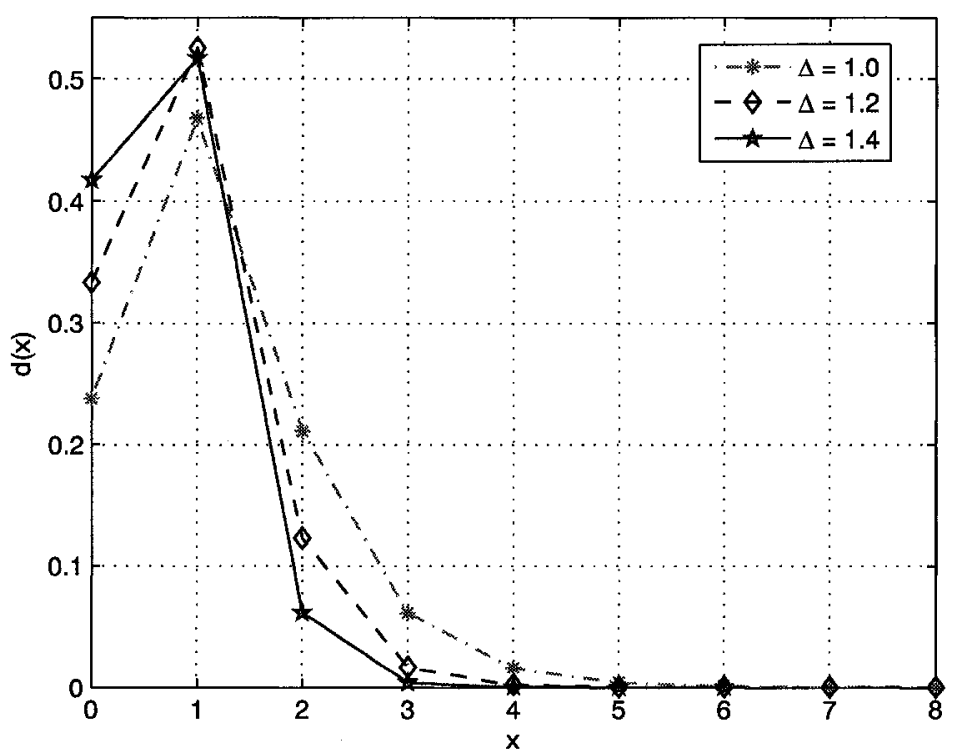

Figure 5.7: The pmf of $\bar{D}$ vs. $\Delta(M=8, p=0.25)$ 


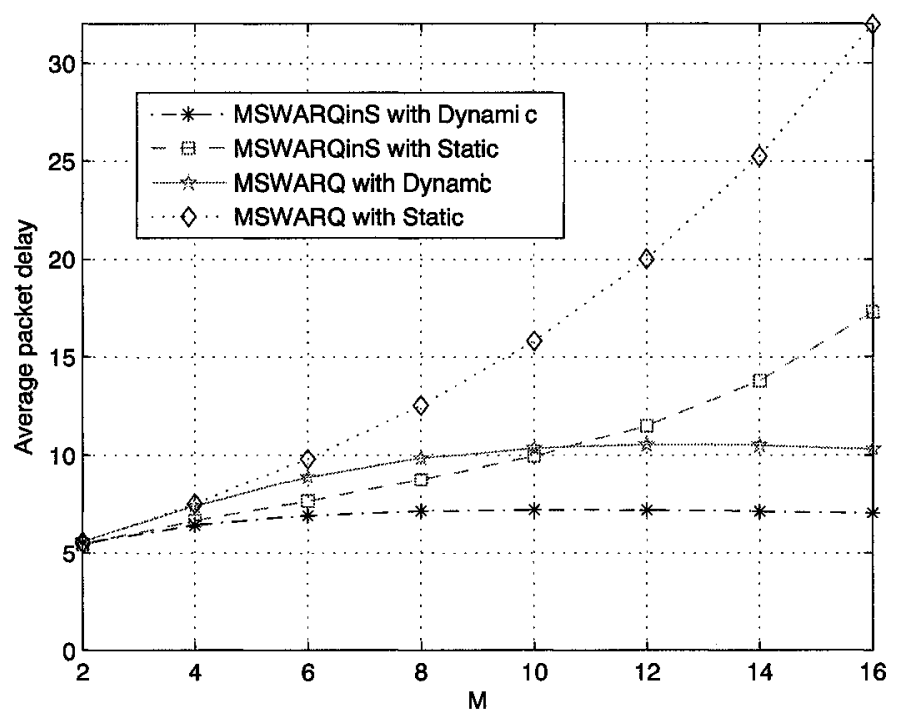

Figure 5.8: Average Packet Delay vs. $M(\Delta=1.2, p=0.25)$

Values of the average packet delay are plotted in Figure 5.8 for $p=0.25, \Delta=1.2$ and $M$ varying from 2 to 16 . As we expect, the average packet delay with the dynamic scheduling is always smaller than that with the static scheduling. The difference of the average delay between the two scheduling policies is small for a small value of $M$, and becomes larger and larger with the increase of $M$. With the dynamic scheduling, the average packet delay increases at first and then reaches a maximum delay value, which shows that the number of parallel channels has only an insignificant impact on the average packet delay when the dynamic scheduling is applied. The last observation from Figure 5.8 is that, with the dynamic scheduling, the mean packet delay of MSW-ARQ-inS is never larger than that of MSW-ARQ. This shows that MSW-ARQ-inS outperforms MSW-ARQ in terms of the mean packet delay performance, which is consistent with the result in [25] under the throughput criterion. 


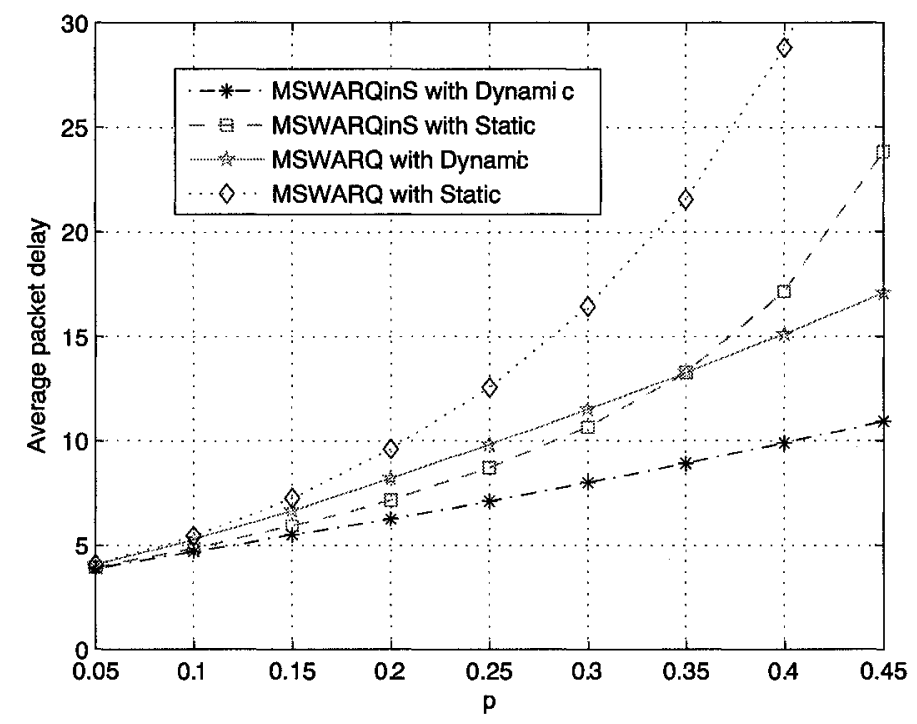

Figure 5.9: Average Packet Delay vs. $p(\Delta=1.2, M=8)$

In Figure 5.9, we plot the average packet delay for $M=8, \Delta=1.2$, and $p$ varying from 0.05 to 0.45 . The average packet delay increases as $p$ does. Similar to the observations from Figure 5.8, for both MSW-ARQ and MSW-ARQ-inS, the packet delay performance with the dynamic scheduling is better than that with the static scheduling; for the dynamic scheduling, MSW-ARQ-inS outperforms MSW-ARQ in terms of the mean packet delay.

Values of the average packet delay are shown in Figure 5.10 for $M=8, p=0.25$ and $\Delta$ varying from 1.1 to 1.7. As observed in Figure 5.8 and Figure 5.9, MSW-ARQ-inS with the dynamic scheduling achieves the best delay performance in all four scenarios. Moreover, when the dynamic scheduling is used, the mean packet delay for both MSWARQ and MSW-ARQ-inS decreases with the increase of $\Delta$, while the corresponding delay with the static scheduling increases with $\Delta$. This is because, the greater the variance 


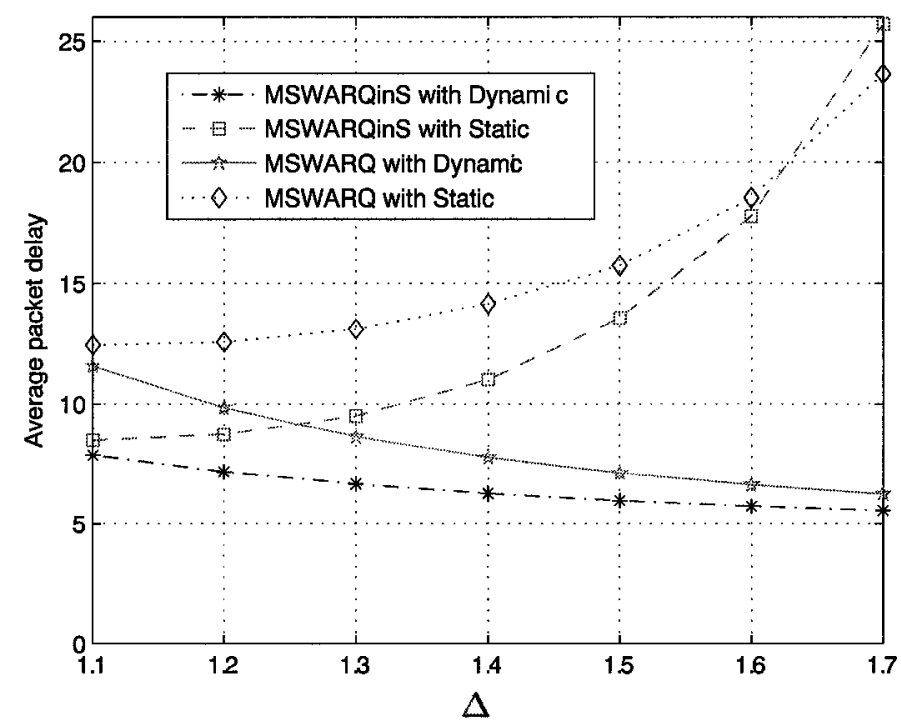

Figure 5.10: Average Packet Delay vs. $\Delta(M=8, p=0.25)$

in the error rates is, the smaller the error rates of the first few channels are. (For instance, in Figure 5.10, the error rates of channels 1 to 4 when $\Delta=1.2$ are smaller than the corresponding ones when $\Delta=1.1$.) Intuitively, the packets transmitted over the first few channels have a larger probability to be correctly received (and delivered to the upper layer), which results in a smaller possibility for the other packets to be retransmitted (for MSW-ARQ) or queued in the resequencing buffer (for MSW-ARQ-inS) with the dynamic scheduling. Therefore, the total number of retransmissions of a packet is reduced, and so is the packet delay.

To verify if the above observation made from time-invariant channels is also valid for time-varying channels, we simulate the multichannel SW-ARQ models for multiple timevarying channels and plot the simulation results of the average packet delay in Figure 5.11. In the plot, $M=8$ and the error rates of these channels change over time based on a two- 


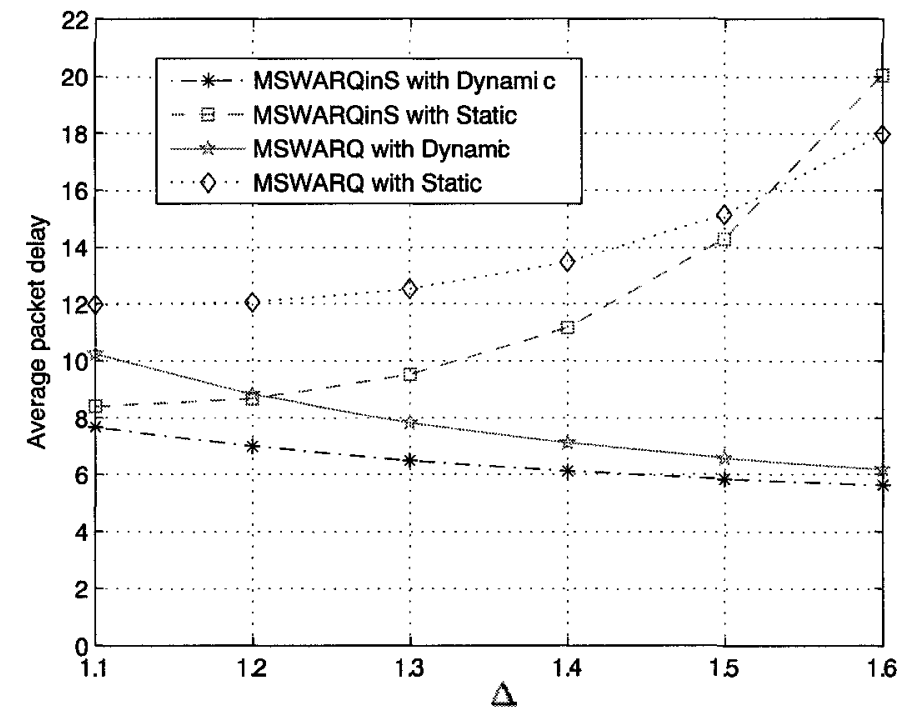

Figure 5.11: Average Packet Delay for Time-Varying Channels

state Markov chain with the transition matrix

$$
\left[\begin{array}{ll}
0.8 & 0.2 \\
0.3 & 0.7
\end{array}\right] .
$$

Each state in the state space of the Markov chain corresponds to a vector of $M$ error rates with a ratio $\Delta$ of any two consecutive error rates in the vector. We assume that the overall average error rate of all channels is $p=0.25$. However, the average error rate of the $M$ error rates in the vector corresponding to a state can be different, which is assumed to be 0.2 and 0.325 , respectively, for the two states in our example. From Figure 5.11, it is clear that the average packet delay for the dynamic scheduling decreases with $\Delta$, from which we conclude that the mean packet delay of both MSW-ARQ and MSW-ARQ-inS with the dynamic scheduling shortens from the growth of the variance in the error rates. We expect that the observation is also valid in general. 


\subsection{Summary}

In this chapter, we derived the probability mass function of the packet delay for MSWARQ and MSW-ARQ-inS with the dynamic scheduling. Based on the analysis, we computed numerically the distribution function for selected values of the number of channels and the error rates for both models. We observed that the probability that a packet is delivered to the upper layer without experiencing any retransmission decreases with the increase of either the number of channels, or the average error rate of all channels, or the variance in the error rates. Moreover, we compared the performance of MSW-ARQ and MSW-ARQ-inS in terms of the mean packet delay and investigated the impact of packet scheduling policies on the delay performance numerically, and through simulation. We observed that:

1. with the dynamic scheduling, MSW-ARQ-inS always outperforms MSW-ARQ;

2. when the dynamic scheduling is applied, the mean packet delay of both models increases with the average error rate but decreases as the variance in the error rates increases, and the number of parallel channels has only an insignificant impact on the mean packet delay;

3. for both models, the dynamic scheduling always outperforms the static scheduling in terms of the mean packet delay performance,

which corroborates the result of Ding and Rice [25] under the throughput criterion. 


\section{Chapter 6}

\section{Conclusion and Future Work}

In this chapter, we conclude the thesis research in Section 6.1 and suggest possible future work in Section 6.2.

\subsection{Conclusion}

In this thesis, we developed two stochastic systems, which are adaptive to real-life implementations and mathematically tractable, for modeling SW-ARQ over multiple parallel channels. By assuming the channel errors are time-invariant, we performed a probabilistic analysis of both models with the dynamic scheduling. For multiple channels of time-varying error rates, we studied the MSW-ARQ-inS model by deriving the probability generating function of the resequencing buffer occupancy. We investigated the impact of system parameters on the mean value performance of both models and conducted performance comparisons of the two packet transmission scheduling policies numerically, and through simulation. 


\subsection{Future Work}

The following is a list of possible future work of this research.

- For the MSW-ARQ-inS model over channels of time-varying error rates, statistical properties of the resequencing buffer occupancy have been obtained in this thesis. Statistical properties of the resequencing delay and the packet delay are of interest to be investigated.

- The models studied in this thesis can be generalized to allow the transmitter to send more than one copy of a packet either simultaneously over multiple channels or when channels are idle. It is necessary to conduct performance comparisons between the generalized models and the original one.

- It is challenging to evaluate the performance of SR-ARQ over multiple channels of time-varying channel error rates, and we expect that the modeling technique and analysis approach used in this thesis can be applied to the analysis of multichannel SR-ARQ.

- Another extension of the research in this thesis is to evaluate the performance of ARQ protocols over multiple channels with both different transmission rates and different error rates. 


\section{Appendix A}

\section{Literature on Packet Resequencing}

In packet data networks, it often happens that packets (the data units transmitted) depart from the receiver in a different order of their arriving at the transmitter due to the randomness of packets' transmission time. In such a system, however, packets are often required to leave the receiver in the same order as they arrived at the transmitter, which is referred to as in-sequence delivery. This can be accomplished by providing a resequencing buffer at the receiver to control the departure of packets. Such a system meeting the packet resequencing requirement, illustrated in Figure A.1 [9], is called a resequencing network. The network includes three components: disordering network, resequencing buffer, and queueing network. Packets arriving at the disordering network are numbered in a numerical sequence. For instance, the sequence $C_{1}, C_{2}, C_{3}, \cdots$ represents a sequence of arriving packets, where $C_{n}$ is the $n^{\text {th }}$ packet arriving at the disordering network. Because of the random nature of sojourn times of packets in the disordering network, packet $C_{n}$ may leave the disordering network before some packet $C_{k}$ with $k<n$. In this case, $C_{n}$ is an out-of-sequence packet. A packet $C_{n}$ is deliverable if it is correctly received and all 


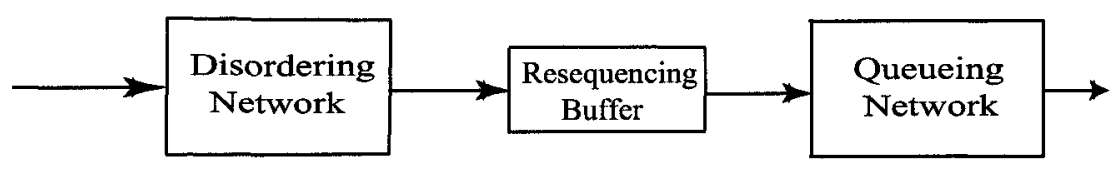

Figure A.1: A General Resequencing Network

packets $C_{k}$, for $k<n$, have also been correctly received. In order to make packets enter the queueing network in the same order as they arrived at the disordering network, the resequencing buffer sends only deliverable packets to the queueing network. For resequencing analysis in the context of the resequencing network, delay in the resequencing buffer (referred to as the resequencing delay) and a number of packets waiting in the resequencing buffer (referred to as the resequencing buffer occupancy) have been the key performance metrics often used in the literature.

By assuming different queueing models in the disordering network component of the resequencing network, several studies have been reported for analysis of the resequencing buffer. Kamoun, Kleinrock and Muntz [48] used an $M / M / \infty$ queue to represent the disordering network, and analyzed the distribution functions of the resequencing buffer occupancy and the resequencing delay. Harrus and Plateau [43] extended the analysis in [48] by replacing the $M / M / \infty$ queue with an $M / G / \infty$ queue. Baccelli, Gelenbe and Plateau [10] further generalized the disordering network to be a $G I / G / \infty$ queue and analyzed the distribution of the packet delay, which includes delays in all three components of the resequencing network, via the factorization method. In this paper, a Markov chain $\left\{Y_{n}\right\}_{n \geq 1}$, in which $Y_{n}$ is the packet delay of packet $C_{n}$, has been constructed, and, under some condition, its stationary distribution function has been obtained.

Resequencing studies in the literature have addressed the case where the disordering 


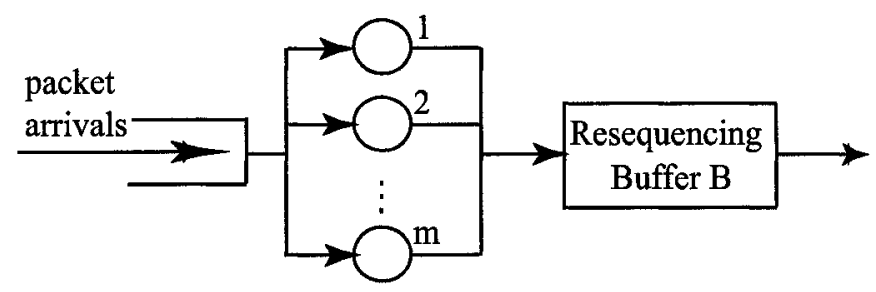

Figure A.2: The Disordering Network with Finite Servers

network consists of a finite number of either homogeneous or heterogenous servers, illustrated in Figure A.2. Bharath-Kumar and Kermani [16] analyzed the mean resequencing buffer occupancy and the mean resequencing delay for an $M / M / m$ queue with the same service rate in the disordering network. Yum and Ngai [100] derived the distribution function of the resequencing delay for the servers in an $M / M / m$ queue having different service rates. Iliadis and Lien [45] investigated the impact of packet transmission scheduling policies on the mean resequencing delay performance for an $M / M / 2$ queue in the disordering network, and an optimal scheduling policy was identified in [57]. The resequencing delay and the packet delay were studied in [46] and [42], respectively. Liu and Towsley [59] identified an optimal packet scheduling policy for a $G I / G / m$ queue in the disordering network. Jean-Marie [47] studied the resequencing delay when the disordering network consists of two independently identical $M / M / 1$ queues, shown in Figure A.3. In this paper, packets are assumed to have two classes and arrive according to two independent Poisson processes. An incoming class $i$ packet joins the first queue with a probability $p_{i}$ or the second queue otherwise, and packets only with the same class are re-ordered in the resequencing buffer. In steady state, the distribution function of the resequencing delay was derived.

Resequencing studies were also conducted for a particular network protocol, such as 


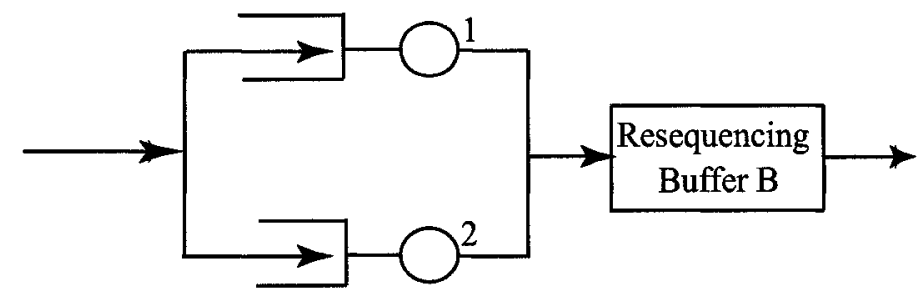

Figure A.3: The Disordering Network with Switched Input

SR-ARQ. In [71] and [78], the resequencing analysis was conducted in the scenarios where the transmitter communicates with a single receiver and multiple receivers, respectively. In the case of the transmitter communicating a single receiver, an $N$-dimensional ( $N$ is known as the number of time units of a packet's round-trip time or called the window sizes) Markov chain $\left\{\left(W_{t}^{1}, \cdots, W_{t}^{N}\right): t=1,2, \cdots\right\}$ was constructed, where it was proved that $W_{t}^{1}-N$ is the size of the resequencing buffer at time $t$, from which the distribution function of the resequencing buffer occupancy in equilibrium regime was derived. Kim and Krunz [49] used the Markov chain $\left\{\left(W_{t}^{1}, \cdots, W_{t}^{N}\right): t=1,2, \cdots\right\}$ to approximate the mean resequencing delay in the case that the channel error rate is timevarying (e.g., an on-off Markov channel model). Xia and Tse [99] considered a SR-ARQ model where the propagation delay of a packets is random and the resequencing buffer is of a limited size, and found an asymptotic tail probability of the resequencing buffer occupancy for some given distribution functions of the propagation delay. In [55], a priority queueing model is used to analyze the mean resequencing delay for SR-ARQ with a Bernoulli arrival process. 


\section{Bibliography}

[1] 3GPP Technical Specification, "TS 25.321 V7.6.0, Medium Access Control (MAC) Protocol Specification (Release 7)," Radio Access Network, 2007.

[2] 802.11g, "Part 11: Wireless LAN Medium Access Control (MAC) and Physical Layer (PHY) specifications: Amendment 4, Further Higher Data Rate Extension in the $2.4 \mathrm{GHz}$ Band," IEEE Standard, 2003.

[3] 802.16, "Part 16: Air Interface for Fixed Broadband Wireless Access Systems," IEEE Standard for Local and Metropolitan Area Networks, 2004.

[4] J. Abate, and W. Whitt, "Numerical Inversion of Probability Generating Functions," Operations Research Letters, vol. 12, no. 4, pp. 245-251, 1992.

[5] A.A. Abouzeid, S. Roy, and M. Azizoglu, "Comprehensive Performance Analysis of a TCP Session over a Wireless Fading Link with Queueing," IEEE Transactions on Wireless Communications, vol. 2, no. 2, pp. 344-356, 2003.

[6] M.E. Anagnostou, and E.N. Protonotarios, "Performance Analysis of the Selective Repeat ARQ Protocol," IEEE Transactions on Communications, vol. 34, no. 2, pp. 127-135, 1986. 
[7] J. Arauz, and P. Krishnamurthy, "Markov Modeling of 802.11 Channels," in Proceedings of IEEE 58th Vehicular Technology Conference, Orlando, Florida, vol. 2, pp. 771-775, 2003.

[8] F. Babich, and G. Lombardi, "On Verifying a First-Order Markovian Model for the Multi-threshold Success/Failure Process for Rayleigh Channel,' In Proceedings of the IEEE International Symposium on Personal, Indoor and Mobile Radio Communications, PIMRC 1997, Helsinki, Finland, pp. 12-16, 1997.

[9] F. Baccelli, and A.M. Makowski, "Synchronization in Queueing Systems," in Stochastic Analysis of Computer and Communication Systems, H. Takagi, ED. 57-129, North-Holland, Amsterdam, 1990.

[10] F. Baccelli, E. Gelenbe, and B. Plateau, "An End-to-End Approach to the Resequencing Problem," Journal of the Association for Computing Machinary, vol. 31, no. 3, pp. $474-485,1984$.

[11] L. Badia, M. Rossi, and M. Zorzi, "SR ARQ Packet Delay Statistics on Markov Channels in the Presence of Variable Arrival Rate," IEEE Transactions on Wireless Communications, vol. 5, no. 7, pp. 1639-1644, 2006.

[12] L. Badia, M. Rossi, and M. Zorzi, "Some Results on the Statistics of Delay Terms in SR ARQ on Markov Channels," In Proceedings of IEEE 2nd International Symposium on Wireless Communication Systems, pp. 64-68, 2005.

[13] L. Badia, M. Rossi, and M. Zorzi, “Queueing and Delivery Analysis of SR ARQ on Markov Channels with Non-instantaneous Feedback," In Proceedings of IEEE GLOBECOM 2005, pp. 3717-3721, 2005. 
[14] A.R.S. Bahai, B.R. Saltzberg, and M. Ergen, Multi Carrier Digital Communications: Theory and Applications of OFDM, New York, Springer, 2004.

[15] R.J.Benice, and A.H. Frey, "An Analysis of Retransmission Systems," IEEE Transactions on Communications, vol. 12, no. 4, pp. 135-145, 1964.

[16] K. Bharath-Kumar, and P. Kermani, "Analysis of a Resequencing Problem in Communication Networks," in Proc. IEEE INFOCOM'83, pp. 303-310, 1983.

[17] Bluetooth SIG Selects WiMedia Alliance Ultra-Wideband Technology for High Speed Bluetooth Applications, Source: http://bluetooth.com/Bluetooth/Press/SIG/, 2006.

[18] Bluetooth SIG, Specification of the Bluetooth System (Version 2.0 + EDR), Source: http://bluetooth.com/, 2004.

[19] P. Bremaud, Markov Chains: Gibbs Fields, Monte Carlo Simulation, and Queues, Springer-Verlag, 1998.

[20] H. Bruneel, and M. Moeneclaey, "On the Throughput Performance of Some Continuous ARQ Strategies with Repeated Transmissions," IEEE Transactions on Communications, vol. COM-34, no. 3, pp. 244-249, 1986.

[21] J.-F. Chang, and T.-H. Yang, "Multichannel ARQ Protocols," IEEE Transactions on Communications, vol. 41, no. 4, pp. 592-598, 1993.

[22] R.W. Chang, "Synthesis of Band-Limited Orthogonal Signals for Multichannel Data Transmission," Bell System Technical Journal, vol. 45, pp. 1775-1796, 1966. 
[23] S.S.L. Chang, "Theory of Information Feedback Systems," IEEE Transactions on Information Theory, vol. 2, no. 3, pp. 29-40, 1956.

[24] Z. Ding, and M. Rice, “Type-I Hybrid-ARQ Using MTCM Spatio-temporal Vector Multichannel Communications", in Proceedings of IEEE International Conference on Communications, pp. 4:2758-2762, 2003.

[25] Z. Ding, and M. Rice, "Throughput analysis of ARQ protocols for parallel multichannel communications," in Proceedings of IEEE GLOBECOM, pp. 1279$1283,2005$.

[26] Z. Ding, "ARQ techniques for MIMO communication systems," Ph.D. thesis, Department of Electrical and Computer Engineering, Brigham Yong University, 2006.

[27] Z. Ding, and M. Rice, "ARQ Error Control for Parallel Multichannel Communications," IEEE Transactions on Wireless Communications, vol. 5, no. 11, pp. 3039-3044, 2006.

[28] E.O. Elliott, "Estimates of Error Rates for Codes on Burst-Noise Channels," Bell System Technical Journal, vol. 42, no. 5, pp. 1977-1997, 1963.

[29] R. Fantacci, "Performance Evaluation of Some Efficient Stop-and-Wait Techniques," IEEE Transactions on Communications, vol. 40, no. 11, pp. 1665-1669, 1992. 
[30] R. Fantacci, "Queueing Analysis of the Selective Repeat Automatic Repeat Request Protocol Wireless Packet Networks," IEEE Transactions on Vehicular Technology, vol. 45, no. 2, pp. 258-264, 1996.

[31] R. Fantacci, "Mean Packet Delay Analysis for the Selective Repeat Automatic Repeat Request Protocol with Correlated Arrivals and Deterministic and Nondeterministic Acknowledgement," Telecommunication Systems, no. 9, pp. 41-57, 1998.

[32] G. Fayolle, E. Gelenbe, and G. Pujolle, "An Analytic Evaluation of the Performance of the "Send and Wait" Protocol," IEEE Transactions on Communications, vol. 26, no. 3, pp. 313-319, 1978.

[33] I. Forkel, H. Klenner, and A. Kemper, "High Speed Downlink Packet Access (HSDPA) - Enhanced Data Rates for UMTS Evolution," Computer Networks: The International Journal of Computer and Telecommunications Networking, vol. 49, no. 3, pp. 325-340, 2005.

[34] B.D. Fritchman, "A Binary Channel Characterization using Partitioned Markov Chains," IEEE Transactions on Information Theory, vol. 13, no. 2, pp. 221-227, 1967.

[35] S. Fujii, Y. Hayashida, and M. Komatu, "Exact Analysis of Delay Performance of Go-Back-N ARQ Scheme over Multiple Parallel Channels," Electronics and Communications in Japan, part 1, vol. 84, no. 9, pp. 27-41, 2001.

[36] F.C. Fujiwara, M. Kasahara, K. Yamashita, and T. Namekawa, "Evaluations of Error Control Techniques in both Independent-Error and Dependent-Error Chan- 
nels," IEEE Transactions on Communications, vol. COM-26, no. 6, pp. 785-794, 1978.

[37] H.E. Gamal, G. Caire, and M.O. Damen, "The MIMO ARQ Channel: DiversityMultiplexing-Delay Tradeoff," IEEE Transactions on Information Theory, vol. 52, no. 8, pp. 3601-3621, 2006.

[38] H.E. Gamal, G. Caire, and M.O. Damen, "The Diversity-Multiplexing-Delay Tradeoff in MIMO ARQ Channels," in Proceedings of IEEE ISIT 2005, pp. $1823-1827,2005$.

[39] A. Ghosh, D.R.Wolter, J.G. Andrews, and R. Chen, "Broadband wireless access with WiMax/8O2.16: Current Performance Benchmarks and Future Potential," IEEE Communication Magazine, vol. 43, no. 2, pp. 129-136, 2005.

[40] A. Ghosh, B. Classon, M. Cudak, and L.A. Jalloul, "Multichannel Stop and Wait ARQ Communication Method and Apparatus," United States Patent No. 7,065,068. Available: http://www.uspto.gov/patft/index.html.

[41] E.N. Gilbert, "Capacity of a Burst-Noise Channel," Bell System Technical Journal, vol. 39, no. 5, pp. 1253-1266, 1960.

[42] N.R. Gogate, and S.S. Panwar, "Assigning Customers to Two Parallel Servers with Resequencing," IEEE Communications Letters, vol. 3, no. 4, pp. 119-121, 1999.

[43] G. Harrus, and B. Plateau, "Queueing analysis of a reordering issue," IEEE Transactions on Software Engineering, vol. SE-8, no. 2, pp. 113-123, 1982. 
[44] T. Hu, D. Afshartous, and G. Young, "Parallel Stop and Wait ARQ in UMTS Peformance and Modeling," in Proceedings of the 2004 World Wireless Congress, San Francisco, CA, 2004.

[45] I. Iliadis, and L.Y.-C. Lien, "Resequencing Delay for a Queuing System with Two Heterogeneous Servers under a Threshold-type Scheduling," IEEE Transactions on Communications, vol. 36, no. 6, pp. 692-702, 1988.

[46] I. Iliadis, and L.Y.-C. Lien, "Resequencing Control for a Queuing System with Two Heterogeneous Servers," IEEE Transactions on Communications, vol. 41, no. 6, pp. 951-961, 1993.

[47] A. Jean-Marie, "Load Balancing in a System of Two Queues with Resequencing," in Proc. the 12th International Symposium on Computer Performance Modelling, Measurement and Evaluation, pp. 75-88, 1987.

[48] F. Kamoun, L. Kleinrock, and R. Muntz, "Queueing analysis of the reordering issue in a distributed database concurrency control mechanism," in Proc. the 2nd International Conference on Distributed Computing Systems, pp. 13-23, 1981.

[49] J.G. Kim, and M.K. Krunz, "Delay Analysis of Selective Repeat ARQ for a Morkovian Source over a Wireless Channel," IEEE Transactions on Vehicular Technology, vol. 49, no. 5, pp. 1968-1981, 2000.

[50] T. Koike, M. Murata, and S. Yoshida, "Hybrid ARQ scheme suitable for coded MIMO transmission," in Proceedings of IEEE International Conference on Communications, pp. 5:1919-1923, 2004. 
[51] A. Konheim, "A Queueing Analysis of Two ARQ Protocols," IEEE Transactions on Wireless Communications, vol. 28, no. 7, pp. 1004-1014, 1980.

[52] L.B. Le, E. Hossain, and A.S. Alfa, "Delay statistics and throughput performance for multi-rate ireless networks under multiuser diversity," IEEE Transactions on Wireless Communications, vol. 5, no. 11, pp. 3234-3243, 2006.

[53] J. Li, and Y.Q. Zhao, "Resequencing Analysis of Stop-and-Wait ARQ for Parallel Multichannel Communications," accepted by IEEE/ACM Transactions on Networking.

[54] J. Li, and Y.Q. Zhao, "Packet Delay Analysis for Multichannel Communication Systems with MSW-ARQ," submitted to Performance Evaluation.

[55] J. Li, and Y.Q. Zhao, "Analysis of Resequencing Delay in a Priority Queueing Model with Delayed Feedbacks,"' submitted to ICOIN 2009.

[56] S. Lin, D.J. Costello, and M.J. Miller, “Automatic-repeat-request Error-control Schemes," IEEE Communication Magazine, vol. 22, no. 12, pp. 5-17, 1984.

[57] W. Lin, and P.R. Kumar, "Optimal Control of a Queuing System with Two Heterogeneous Servers," IEEE Transactions on Automatic Control, vol. AC-29, pp. 696-703, 1984.

[58] S. Lin, and D.J. Costello, Error Control Coding: Fundamentals and Applications, Englewood Cliffs, N.J., Prentice-Hall, 1983.

[59] Z. Liu, and D. Towsley, "Effects of Service Disciplines in G/GI/s Queueing Systems," Technical Report: UM-CS-1992-026, 1992. 
[60] D.L. Lu, and J.F. Chang, "Performance of ARQ Protocols in Nonindependent Channel Errors," IEEE Transactions on Communications, vol. 41, no. 5, pp. 721$730,1993$.

[61] W. Luo, K. Balachandran, S. Nanda, and K.K. Chang, "Delay Analysis of Selective-Repeat ARQ with Applications to Link Adaption in Wireless Packet Data Systems," IEEE Transactions on Wireless Communications, vol. 4, no. 3, pp. 1017-1028, 2005.

[62] M.J. Miller, and S.L. Lin, "The Analysis of Some Selective-Repeat ARQ Schemes with Finite Receiver Buffer," IEEE Transactions on Communications, vol. 29, no. 9, pp. 1307-1315, 1981.

[63] M. Moeneclaey, H. Nruneel, J. Bruyland, and D.Y. Chung, "Throughput Optimization for a Generalized Stop-and-Wait ARQ Scheme," IEEE Transactions on Communications, vol. 34, no. 2, pp. 205-207, 1986.

[64] A.F. Molisch, Wireless Communications, John Wiley \& Sons, England, 2005.

[65] M.D. Munnynck, A. Lootens, S. Wittevrongel, and H. Bruneel, "Transmitter buffer behaviour of Stop-and-Wait ARQ Schemes with repeated transmissions," IEEE Proceedings - Communications, vol. 149, no. 1, pp. 13-17, 2002.

[66] A.V. Nguyen, and M.A. Ingram, "Hybrid ARQ protocols using space-time codes," in Proceedings of 54th IEEE Veh. Tch. Conf., vol. 4, pp. 2364-2368, 2001. 
[67] E.N. Onggosanusi, E.G. Dabak, Y. Hui, and G. Jeong, "Hybrid ARQ transmission and combining for MIMO systems", in Proceedings of IEEE Int. Conf. Commun., vol. 5, pp. 3205-3209, 2003.

[68] S.A. Pawar, et al., "Achieving the DMD Tradeoff of the MIMO-ARQ Channel," in Proceedings of IEEE ISIT 2005, pp. 901-905, 2005.

[69] T.S. Rappaport, Wireless Communications: Principles and Practice, 2nd edition, Prentice Hall, 2002.

[70] J. Redi, et al., "Design and Implementation of a MIMO MAC protocol for ad hoc Networking," in Proceedings of SPIE 2006, Orlando, FL., Vol. 6248, 624802-1$12,2006$.

[71] Z. Rosberg, and N. Shacham, "Resequencing Delay and Buffer Occupancy Under the Selective-Repeat ARQ," IEEE Transactions on Information Theory, vol. 35, no. 1, pp. 166-173, 1989.

[72] Z. Rosberg, and M. Sidi, "Selective-Repeat ARQ: the Joint Distribution of the Transmitter and the Receiver Resequencing Buffer Occupancies," IEEE Transactions on Communications, vol. 38, no. 9, pp. 1430-1438, 1990.

[73] M. Rossi, L. Badia, and M. Zorzi, "SR ARQ Delay Statistics on N-State Markov Channels with Non-Instantaneous Feedback," IEEE Transactions on Wireless Communications, vol. 5, no. 6, pp. 1526-1536, 2006. 
[74] M. Rossi, L. Badia, and M. Zorzi, "On the Delay Statistics of SR ARQ over Markov Channels with Finite Round-Trip Delay," IEEE Transactions on Wireless Communications, vol. 4, no. 4, pp. 1858-1868, 2005.

[75] M. Rossi, and M. Zorzi, "Analysis and Heuristics of the Characterization of Selective-Repeat ARQ Delay Statistics over Wireless Channels," IEEE Transactions on Vehicular Technology, vol. 52, no. 5, pp. 1365-1377, 2003.

[76] H. Samra, and Z. Ding, "New MIMO ARQ Protocols and Joint Detection via Sphere Decoding," IEEE Transactions on Signal Processing, vol. 54, no. 2, pp. $473-482,2006$.

[77] N. Shacham, "Packet Resequencing under Reliable Transport Protocols," in Proceedings of IEEE ICC 1989, 716-723, 1989.

[78] N. Shacham, and D. Towsley, "Resequencing Delay and Buffer Occupancy in Selective Repeat ARQ with Multiple Receivers," IEEE Transactions on Communications, vol. 39, no. 6, pp. 928-937, 1991.

[79] N. Shacham, and B.C. Shin, "A Selective-Repeat-ARQ Protocol for Parallel Channels and Its Resequencing Analysis," IEEE Transactions on Communications, vol. 40, no. 4, pp. 773-782, 1992.

[80] T. Shikama, S. Deno, T. Watanabe, and T. Mizuno "Delay Analysis of the Selective-Repeat ARQ Protocol with the Per-Flow Resequencing Schemes," IPSJ Digital Courier, vol. 2, pp. 81-93, 2006. 
[81] T. Shikama, and T. Mizuno "Resequencing Schemes for Selective-Repeat ARQ and Their Performance," in Proceedings of IEEE 19th International Conference on Advanced Information Networking and Applications, vol. 2, pp. 491-494, 2005 .

[82] M. Sima, Y. Zhou, and W. Zhang, "Joint Selective Repeat Automatic Repeat Request (SR-ARQ) Protocols Based on Multiple Markov Channels,' Journal of Shanghai Jiaotong University, vol. 38 sup., 2004, pp. 57-62.

[83] W. Stallings, Data and Computer Communications, 6th edition, Prentice-Hall Inc., New Jersey, 2000.

[84] N. Sugimachi, Y. Hayashida, Y. Yoshida, "Delay Performances of Tandem Stopand-Wait ARQ Scheme," Electronics and Communications in Japan (Part I: Communications), vol. 73, no. 11, pp. 65-72, 1990.

[85] H. Sun, J.H. Manton, and Z. Ding, "Progressive Linear Precoder Optimization for MIMO Packet Retransmissions," IEEE Journal on Selected Areas in Communications, vol. 24, no. 3, pp. 448-456, 2006.

[86] H. Sun, H. Samra, Z. Ding, and J.H. Manton, "CONSTRAINED CAPACITY OF LINEAR PRECODED ARQ IN MIMO WIRELESS SYSTEMS," in Proceedings of IEEE International Conference on Acoustics, Speech, and Signal Processing, pp. III: 425-428, 2005.

[87] F. Swarts, and H.C. Ferreira, "Markov Characterization of Channels with Soft Decision Outputs," IEEE Transactions on Communications, vol. 41, no. 5, pp. $678-682,1993$. 
[88] A.L. Toledo, and X. Wang, “TCP Performance over Wireless MIMO Channels with ARQ and Packet Combining," IEEE Transactions on Mobile Computing, vol. 5, no. 3, pp. 208-223, 2006.

[89] D. Towsley, "A Statistical Analysis of ARQ Protocols Operating in a Nonindependent Error Environment," IEEE Transactions on Communications, vol. COM29, no. 7, pp. 971-981, 1981.

[90] D. Towsley, and J. Wolf, “On the Statistical Analysis of Queue Lengths and Waiting Times for Statistical Multiplexers with ARQ Retransmission Schemes," IEEE Transactions on Communications, vol. 27, no. 4, pp. 693-702, 1979.

[91] TSG-RAN Working Group 1, "Clarification on Dual-Channel Stop-and-Wait HARQ," Source: Motorola, Boston, USA, R1-01-0048, 2001.

[92] S.D. Vuyst, "Analysis of Discrete-Time Queueing Systems with Multidimensional State Space," Ph.D. thesis, Gent University, 2006.

[93] H.S. Wang, and N. Moayeri, "Finite State Markov Channel - A Useful Model for Radio Communication Channels," IEEE Transactions on Vehicular Technology, vol. 44, no. 1, pp. 163-171, 1995.

[94] H.S. Wang, and P.C. Chang, "On Verifying the First-Order Markovian Assumption for a Rayleigh Fading Channel Model," IEEE Transactions on Vehicular Technology, vol. 45, no. 5, pp. 353-357, 1996.

[95] S.B. Wicker, Error Control Systems for Digital Communication and Storage, Prentice Hall, 1995. 
[96] J.H. Winters, “On the Capacity of Radio Communication Systems with Diversity in a Rayleigh Fading Environment," IEEE Journal on Selected Areas in Communications, vol. SAC-5, no. 5, pp. 871-878, 1987.

[97] J.M. Wozencraft and M. Horstein, "Digitalized Communication over Two-way Channels," Fourth London Symposium on Information Theory, London, U.K., 1960.

[98] W.-C. Wu, S. Vassiliadis, and T.-Y. Chung, "Performance Analysis of MultiChannel ARQ Protocols," in Proceedings of the 36th Midwest Symposium on Circuits and Systems, vol. 2, pp. 1328-1331, 1993.

[99] Y. Xia, and D. Tse, "Analysis on Packet Resequencing for Reliable Network Protocols," Performance Evaluation, vol. 61, no. 4, pp. 299-328, 2005.

[100] T.S. Yum, and T.Y. Ngai, "Resequencing of Messages in Communication Networks," IEEE Transactions on Communications, vol. 34, no. 2, pp. 143-149, 1986.

[101] Q. Zhang, and S.A. Kassam, "Finite State Markov Models for Rayleigh Fading Channels," IEEE Transactions on Communications, vol. 47, no. 11, pp. 1688$1692,1999$.

[102] H. Zheng, A. Lozano, and M. Haleem, "Multiple ARQ Processes for MIMO Systems," EURASIP Journal on Applied Signal Processing, 5, pp. 772-782, 2004. 